\title{
WestVirginiaUniversity
}

THE RESEARCH REPOSITORY @ WVU

Graduate Theses, Dissertations, and Problem Reports

2016

\section{Characteristics of bubble behavior in microgravity conditions}

Thilanka Munasinghe

Follow this and additional works at: https://researchrepository.wvu.edu/etd

\section{Recommended Citation}

Munasinghe, Thilanka, "Characteristics of bubble behavior in microgravity conditions" (2016). Graduate Theses, Dissertations, and Problem Reports. 6279.

https://researchrepository.wvu.edu/etd/6279

This Thesis is protected by copyright and/or related rights. It has been brought to you by the The Research Repository @ WVU with permission from the rights-holder(s). You are free to use this Thesis in any way that is permitted by the copyright and related rights legislation that applies to your use. For other uses you must obtain permission from the rights-holder(s) directly, unless additional rights are indicated by a Creative Commons license in the record and/ or on the work itself. This Thesis has been accepted for inclusion in WVU Graduate Theses, Dissertations, and Problem Reports collection by an authorized administrator of The Research Repository @ WVU. For more information, please contact researchrepository@mail.wvu.edu. 


\title{
CHARACTERISTICS OF BUBBLE BEHAVIOR IN MICROGRAVITY CONDITIONS
}

\author{
Thilanka Munasinghe \\ Thesis submitted to the College of Engineering \\ at West Virginia University \\ in partial fulfillment of the requirements \\ for the degree of
}

Master of Science

in

Mechanical Engineering

John Kuhlman, PhD, Committee Chair

Patrick Browning, PhD

Christopher Griffin, PhD

Mechanical and Aerospace Engineering

Morgantown, West Virginia

Spring 2016

Keywords: Microgravity, Pool Boiling

Copyright 2016 West Virginia University 


\section{Abstract \\ CHARACTERISTICS OF BUBBLE BEHAVIOR IN MICROGRAVITY CONDITIONS}

\section{Thilanka Munasinghe}

The main objective of this thesis is to study the characteristics of bubbles in pool boiling under microgravity conditions with and without the influence of a magnetic field. In order to create microgravity conditions, the parabolic path of an aircraft was used for a period of 20-30 seconds, during which time the experiment was conducted. Two cylindrical tanks were used for the boiling process in the experiment. One tank had a permanent magnet attached at the bottom, and the other one was kept as the control. $\mathrm{MnCl}_{2}$ was dissolved in distilled water to make a paramagnetic liquid, and which used in the pool boiling process. The experiment was captured on video simultaneously from two different angles, and later analyzed frame by frame using image processing techniques to obtain the bubble characteristics during the different stages of the boiling process. During this investigation of bubble motion, two main studies were conducted. First, the characteristics of a single bubble were studied, such as: center of the bubble, shape of the bubble (maximum radius and minimum radius in vertical and horizontal direction), vertical and horizontal displacement of the center of the single bubble, rate of change of the position of the bubble coordinates and the vertical component of the selected single bubble velocity. Second, the motion of the bubbles was studied, including the directionality, the velocity and the fluctuation of the size and shape of the bubble was studied. The results from the study revealed that as the strength of the magnetic field reduces, the influence of the magnetic field on bubbles was weakened along the vertical axis of the tank. This phenomenon was visualized graphically with various bubble metrics. 


\section{Acknowledgments}

I would like to take this opportunity to express my gratitude to my research advisor Dr. John Kuhlman for his advice and guidance throughout this research project. I am also immensely grateful to my committee members, Dr. Patrick Browning and Dr. Christopher Griffin for their expert advice and kind support. I would also like to thank Dr. Jaggu Nanduri, Dr. Ismail Celik, Dr. Mary Anne Clarke, Dr. Donald Gray, Dr. Arun Ross, and Dr. Majid Jaridi for their support in many ways to make this thesis a success. I am thankful to my microgravity research team members and my fellow colleagues who worked and supported me in many ways with their generous support.

I am thankful to my parents for all what they have done for me to since the day I was born. I am grateful have a sister and for her kindness. I wish to express my gratitude to my relatives and friends who encouraged me and supported me in many ways. I am blessed with a special person in my life - my wife, for her love, support and care since the day I met her.

Special thanks to our main sponsors: NASA - West Virginia Space Grant Consortium and the Mechanical and Aerospace Engineering Department of West Virginia University for the generous financial support for the microgravity team, as well as the Zero Gravity Cooperation for their support. 


\section{Table of Contents}

Abstract Error! Bookmark not defined.

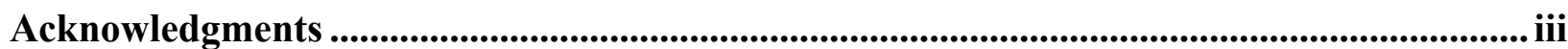

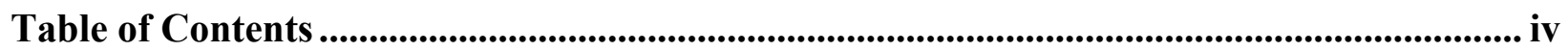

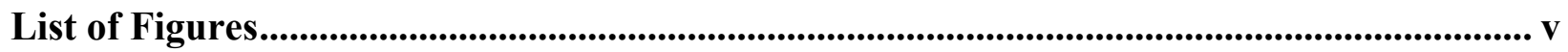

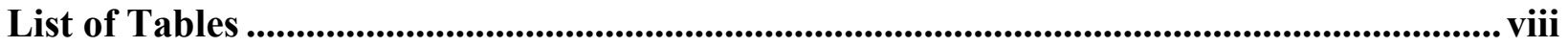

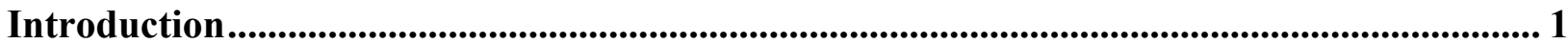

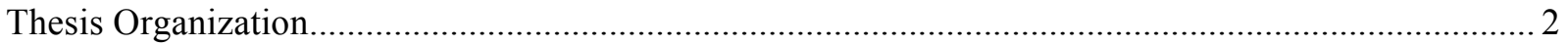

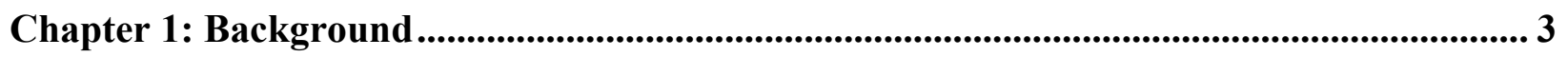

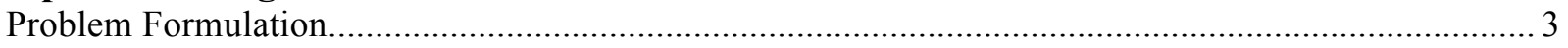

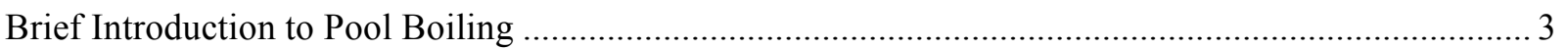

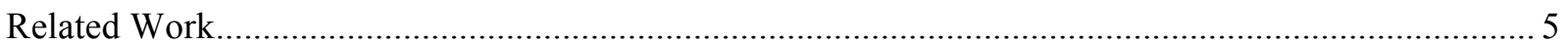

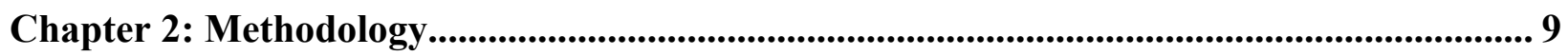

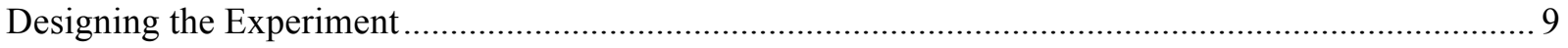

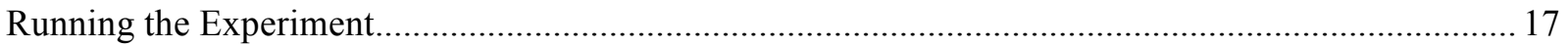

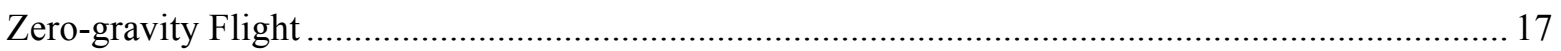

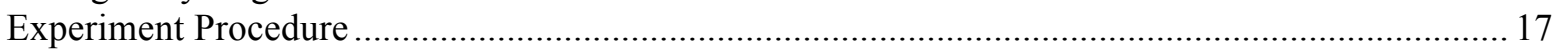

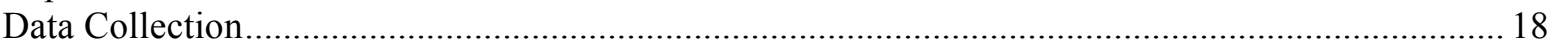

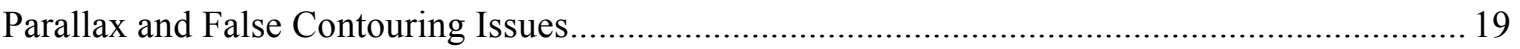

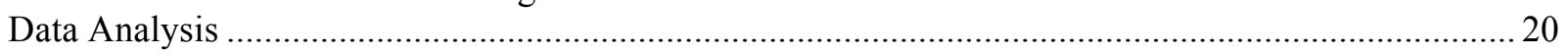

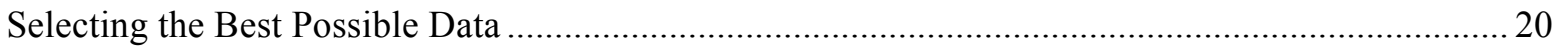

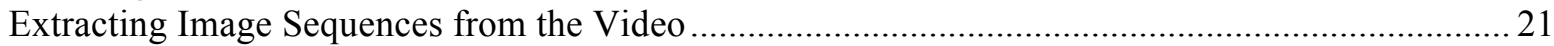

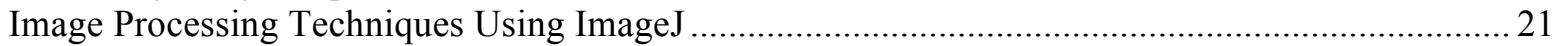

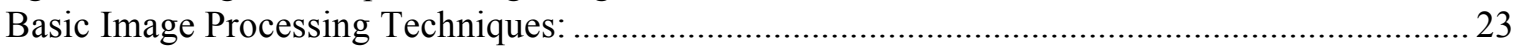

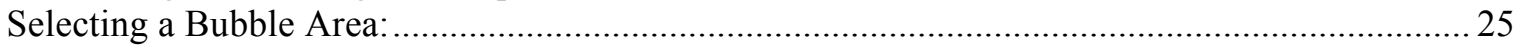

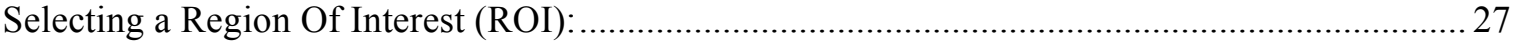

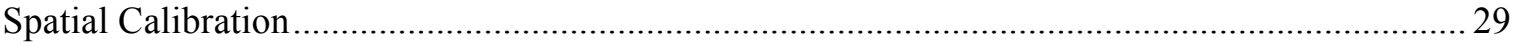

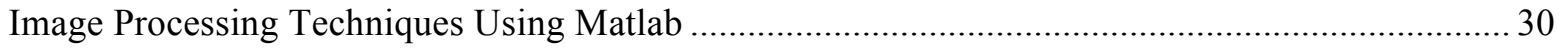

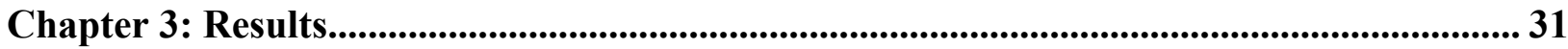

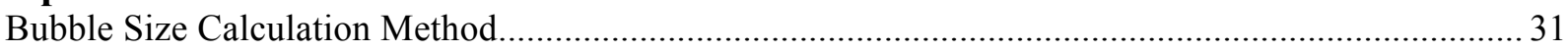

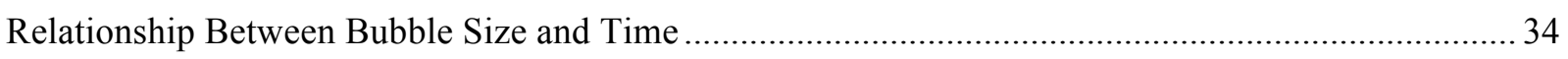

Relationship Between Bubble Radius and Bubble Height............................................................... 37

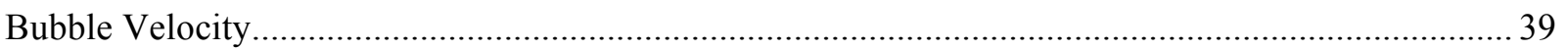

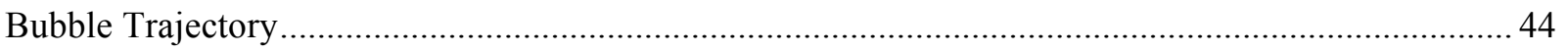

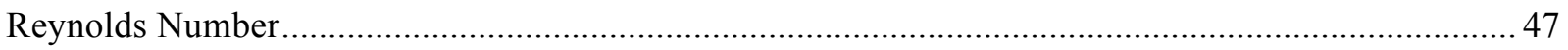

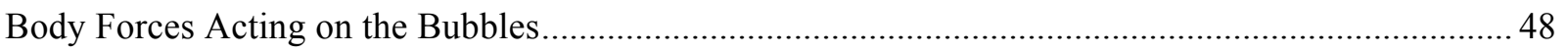

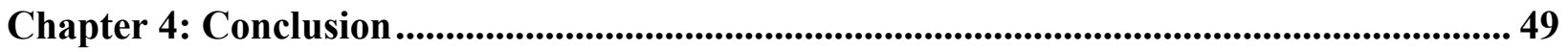

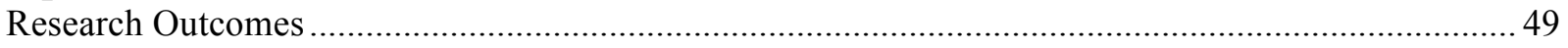

Appendix: Preliminary Methods Used for Image Processing..............................................51

Image Processing by using Matlab ${ }^{\circledR}$ "Image Processing Toolbox" ................................................. 51

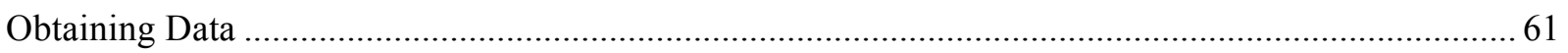




\section{List of Figures}

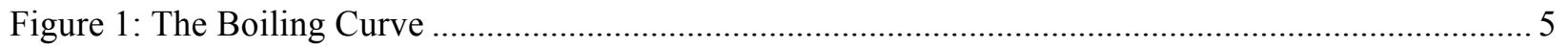

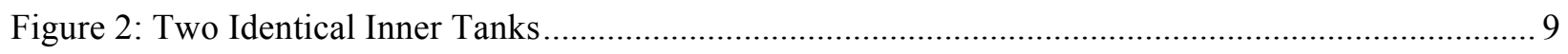

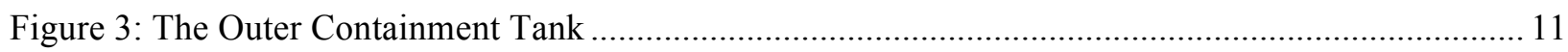

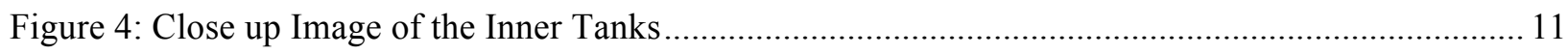

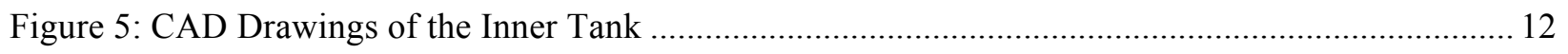

Figure 6: Tanks with Omega HH5010K 4-Channel Thermocouple Readers ......................................... 13

Figure 7: Visual Representation of the Strength of the Magnet Field Along the Radial Distance of the

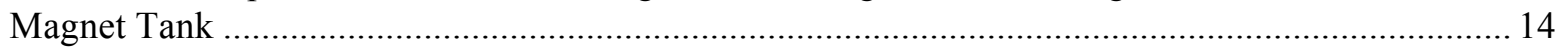

Figure 8: $100 \mathrm{~W}$ Heater on the Base of Magnet Tank and Non-Magnet Tank ...................................... 15

Figure 9: Heater Surface After the Sand Grain Applied .................................................................... 15

Figure 10: Industrial Standard Sand Filters 100 - 120 microns ......................................................... 15

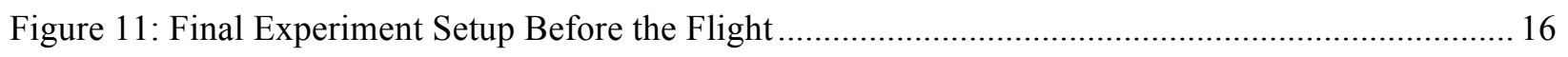

Figure 12: Measuring and Comparing the Known Line Distance on an Image Over Multiple Locations to

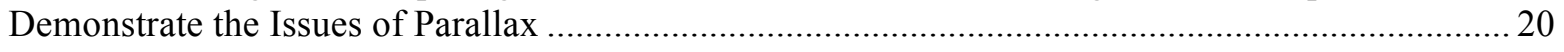

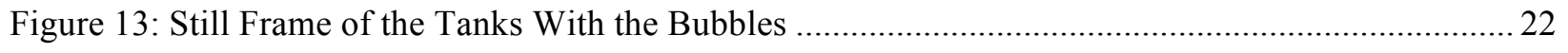

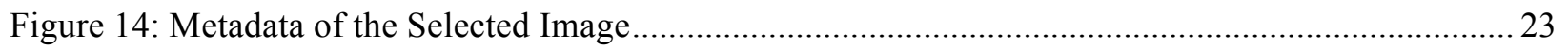

Figure 15: Obtaining the co-ordinates of bubbles along the Major and Minor Axes ............................. 24

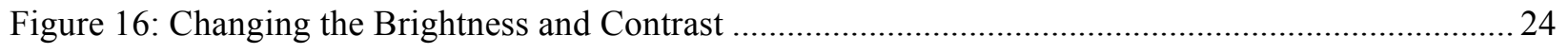

Figure 17: Information Worthy Areas of Some Image Sequences ..................................................... 25

Figure 18: The Original Image and a Selected Bubble From the Image ................................................. 26

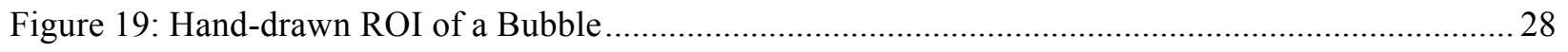

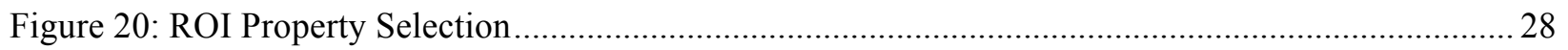

Figure 21: Tank's Flange measured using a Vernier Caliper.................................................................. 29

Figure 22: Set Scale Function Used to calibrate the Tank's Flange ...................................................... 30

Figure 23: Area Calculation using Region of Interest Method for Bubbles; Parabola 34 ........................ 32

Figure 24: Comparison of Bubble Area Calculated as a Circle and as an Ellipse; Parabola 34 ................. 33

Figure 25: Areas of Bubbles with Respect to Time (Magnet Tank); Parabola 35 .................................... 36

Figure 26: Areas of Bubbles with Respect to Time (Non Magnet Tank); Parabola 35 ............................ 36 
Figure 27: Comparison of Average Areas of Bubble Samples Collected from the Tank with and without the Magnet; Parabola 35.

Figure 28: Average Bubble Radii vs. the Height from the bottom of the Tank (Magnet Tank); Parabola 35 . 38

Figure 29: Average Bubble Radii vs. the Height from the bottom of the Tank (Non Magnet Tank); Parabola 35 38

Figure 30: Visual Representation of the Horizontal Velocities of the Bubbles that are Located in Magnet Tank); Parabola 35 .. 40

Figure 31: Vertical Velocity (Magnet Tank); Parabola 35 ................................................................. 41

Figure 32: Horizontal Velocity (Non Magnet Tank); Parabola 35. ......................................................... 41

Figure 33: Vertical Velocity (Non Magnet Tank); Parabola 35 …......................................................... 42

Figure 34: Comparison of the Average Horizontal Velocities in the Two Tanks; Parabola 35. ................ 42

Figure 35: Comparison of Average Vertical Velocity in the Two Tanks; Parabola 35 ........................... 43

Figure 36: Horizontal Distance of Bubbles from Right Wall (Magnet Tank); Parabola 35 ...................... 44

Figure 37: Horizontal Displacement of Bubbles (Non Magnet Tank); Parabola 35 ................................ 45

Figure 38: Vertical Distance of Bubbles from the Heater Surface (Magnet Tank); Parabola 35 .............. 46

Figure 39: Vertical Distance of Bubbles from the Heater Surface (Non Magnet Tank); Parabola 35 ....... 46

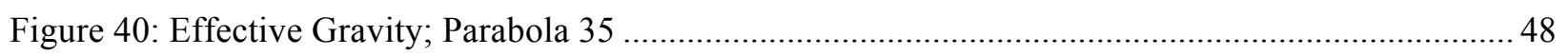

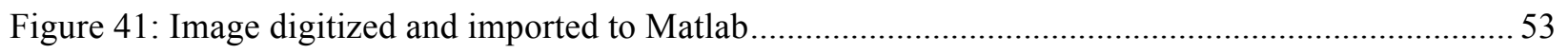

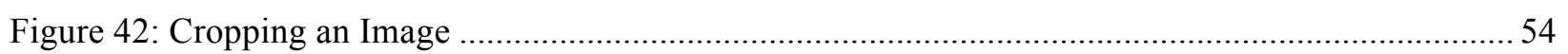

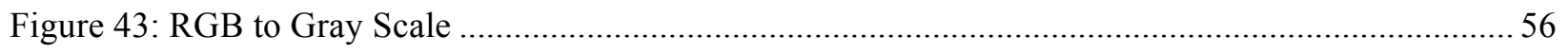

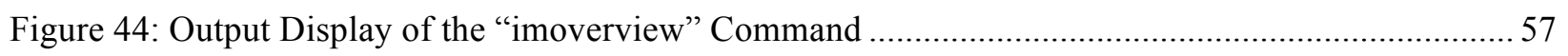

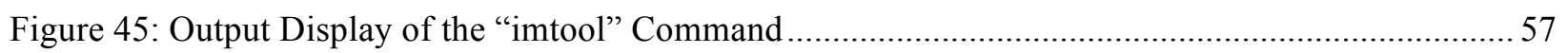

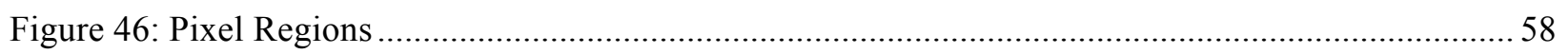

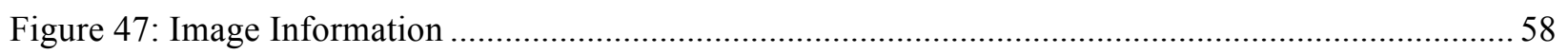

Figure 48: Adjusting the Contrast of the Image with the Histogram .......................................................59

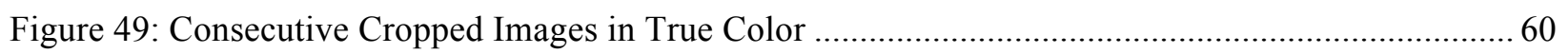

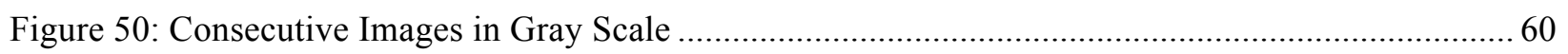

Figure 51: The Two Dimensional Oscillating Path Behavior of the Bubble as it Travers up the Tank in the

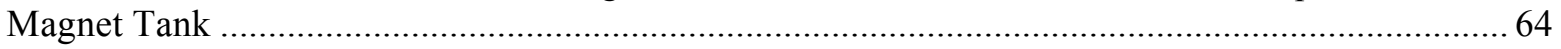

Figure 52:The Vertical Displacement of the Center of the Bubble's As Ii Travers up Long the Magnet Tank.

Figure 53: Difference in the Horizon and Vertical Radii (semi major and semi minor axes) of the Bubble as it Travers up the Tank Under the Influence of the Magnetic Field. ............................................. 66

Figure 54: The Visualization of the Increase of the Bubble Area as it Travers up Along the Magnet Tank 
Figure 55: Resultant Velocity Vector of the Horizontal Velocity Component and the Vertical Velocity Component of the Bubble with Respect to the Position as it Traverse Along the Magnet Tank........ 68

Figure 56: The Visual Representation of the Fluctuations in Vertical and Horizontal Velocity Components as the Bubble Travers upward the Tank

Figure 57: Horizontal and Vertical Radius of the Bubble vs. Frame Number under Magnetic Field ........ 72

Figure 58: Visual Representation of the Size Increase of the Horizontal and Vertical Radii of the Bubble located in the Non-Magnet Tank as it Traverse up the Tank.

Figure 59: Representation of the Increase in Area of Two chosen Bubbles, one Bubble Under the Influence of the Magnetic Field and the Other Bubble, without the Influence of the Magnetic Field as they Traverse Upward the Tank. 


\section{List of Tables}

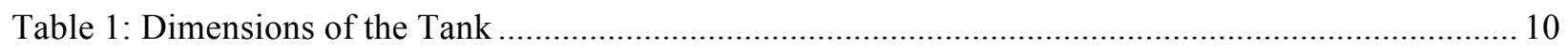

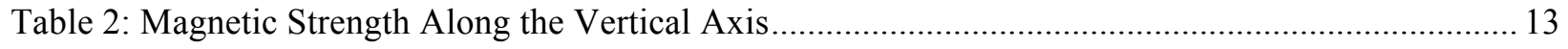

Table 3: Difference Between the drawn line segments........................................................................... 19

Table 4: Percentage Difference Between Circle and Ellipse Areas for Bubble 2 .................................... 34

Table 5: Measure of Central Tendencies of the Bubble Velocities............................................................ 43

Table 6: Characteristic Data of a Bubble from the Tank with the Magnet ............................................... 70

Table 7: Characteristic Data from the Tank without the Magnet............................................................ 71 


\section{Nomenclature}

$\mathrm{a}=$ length of ellipse semimajor axis

$\mathrm{A}_{\text {circle }}=$ Area as a circle

$\mathrm{A}_{\text {ellipse }}=$ Area as an ellipse

$\mathrm{b}=$ Length of ellipse semiminor axis

$B=$ Magnetic Field Strength

$\mathrm{CHF}=$ Critical Heat Flux

fps $=$ frames per second

$g_{\text {effective }}=$ Effective Gravitation force

$R e=$ Reynolds Number

ROI $=$ Region of Interest

$T_{\text {sat }}=$ Saturation Temperature

$T_{\mathrm{w}}=$ Temperature at heating pad surface

$u_{n}=$ Horizontal velocity component

$\mathrm{UI}=$ User Interface

$v_{n}=$ Vertical velocity component

$\rho=$ Density

$\mu_{0}=$ Permeability of free space

$\chi_{s}=$ Magnetic Susceptibility

$\nabla=$ Del Operator

$\Delta t=$ Time difference 


\section{Introduction}

The basic understanding of the way fluids behave under microgravity conditions is imperative in applications such as propulsion, refrigeration, oxygenation systems, and life support systems as the demands of space systems such as communications satellites and manned platforms and missions are expected to grow rapidly. Under normal gravity, bubbles rise due to their buoyancy as the effects of gravity dominate the behavior of fluids. However, under microgravity, such as in Earth orbit, the effects of gravity are removed, and the bubbles more accurately trace the flow of the liquid. In heating applications this can cause the bubbles to remain close to the heating surface resulting in large bubble sizes. A key drawback to this bubble growth is the difficulty of liquid replenishment of the near-wall region, which can result in very small Critical Heat Flux values. This can cause burnout, possibly damaging the surface that needs to be cooled, and is one of the key reasons behind the present day reluctance to incorporate pool boiling in thermal management hardware systems in space applications. Therefore, by understanding the patterns of bubble characteristics that are formed during the pool boiling process we can get a better understanding as to how heat is transferred in microgravity.

In particular, the research outlined in this thesis helps to enable us to ask some very important questions such as: What happens to basic physical, chemical and biological processes in the absence of this very important directional force that is a fundamental force of the universe? What does this teach us about the processes in the universe, on our Earth, and even inside bodies under varying gravitational conditions? Can these insights lead to greater awareness and technological improvements? 


\section{Thesis Organization}

This thesis is organized as follows: Chapter 1 is dedicated to the problem formulation, the underlying theories behind the research presented, as well as the related work elicited from a literature survey. Chapter 2 presents the methodology followed while designing and running the experiment as well as analyzing the data collected from the experiment. Chapter 3 presents the results from the analysis that gives us a better understanding of the bubble characteristics that includes the bubble size, velocity and shape under microgravity conditions in regards to heat transfer, that advances the state of the art of the field. Chapter 4 summarizes the research work conducted for this thesis and outlines possible future directions for this work. Finally, the Appendix includes some of the preliminary analysis work done as part of this thesis using Matlab's image processing toolkit before moving to the more feature-rich ImageJ library that enabled the collection of more accurate data, as discussed in the results of this thesis. 


\section{Chapter 1: Background}

\section{Problem Formulation}

As outlined in the Introduction, boiling is encountered in many engineering fields including space applications. However, the heat transfer characteristics of bubbles in microgravity conditions are not very well understood. Thus, the main objective of this research project was focused on this very important area of study.

In order to create microgravity conditions artificially for the experiment, we conducted the experiment inside the Boeing 727 aircraft from Zero-G Corporation ${ }^{1}$ in a parabolic path, in which the microgravity conditions lasted for a short time period of 20-30 seconds. Pool boiling was generated in two identical tanks: one with a fixed magnet beneath the heater surface, and another without the magnet. The pool boiling process was continued inside the tanks throughout the parabolic maneuver of the aircraft, and was captured simultaneously from two different angles on videos. Magnetic strength of the circular magnet along the vertical axis of tank was also measured to study the influence on bubbles. After capturing all the data a detailed frame-by-frame analysis of bubble characteristics using image-processing techniques was conducted.

The results from this experiment are very promising, and give a very good insight into the bubble characteristics under microgravity conditions as explained in the subsequent chapters of this thesis.

\section{Brief Introduction to Pool Boiling}

Boiling is an evaporation phenomenon that occurs at the solid-liquid interface when the temperature of the surface is higher than the saturation temperature of the liquid contacting the surface. The resulting heat flux depends on the difference between the surface and saturation temperatures. As the heat gets transferred from the heating surface to the fluid natural convection takes place in a gravitational

\footnotetext{
${ }^{1}$ Zero Gravity Corporation, Florida, USA.
} 
field. When the temperature of the fluid reaches its saturation temperature, 'boiling' takes place. From a fundamental point of view, gravity induced convection may mask some important effects, thus microgravity experiments are very useful for basic pool boiling investigations.

Pool boiling is different from convection boiling, and is a mode of boiling in which the heating surface is submerged in a large but finite body of stagnant liquid. The relative motion of the vapor produced and the surrounding liquid near the heating surface is due primarily to the buoyancy effect of the vapor even though the body of the liquid as a whole is essentially at rest. Boiling takes place via bubble nucleation, growth and detachment as explained in (Straub and Johannes, 2001) and is displayed in Figure 1. This shows the average heat flux vs. the difference between the liquid temperature and the saturation temperature.

As shown in Figure 1, the various stages of the pool boiling process is as follows:

- Nucleate Boiling: This stage of the pool boiling process has two parts.

- Liquid Entrainment:

- Due to continuous heating the bubbles are formed at the bottom surface of the tank, and these bubbles move upwards. But they collapse after covering some distance in the liquid. Rising bubbles carry some liquid along with them, and this is known as liquid entrainment.

- Critical Heat Flux (CHF):

- The disturbance caused by liquid entrainment increases the heat transfer coefficient, and thus heat flux also increases. This causes bubbles to form at a faster rate. These vapor bubbles move upwards and merge to form numerous continuous columns of vapor in the liquid. The bubbles in these columns then move upwards to the free surface, where they break up and release their vapor content. Due to this the heat flux attains a maximum value and this stage is known as the CHF stage.

- Transition Boiling:

- When the heat increases beyond the critical point, the heat flux starts decreasing. This is because a large fraction of the heated surface of the vessel is covered with a vapor film. The vapor film acts as an insulator as its thermal conductivity is lower than that of the liquid phase. Thus, the value of heat flux attains its minimum value during this stage and 
is known as the LeidenFrost Point. Because both the nucleate and film boiling takes place during this stage, it is known as the transition boiling stage.

- $\quad$ Film Boiling

- After the transition phase, there is a stable vapor film at the heating surface completely covering it. Because of the high temperatures, significant radiation heat transfer takes place between the heated surface and the liquid through the vapor film. Thus, this stage is known as film boiling.

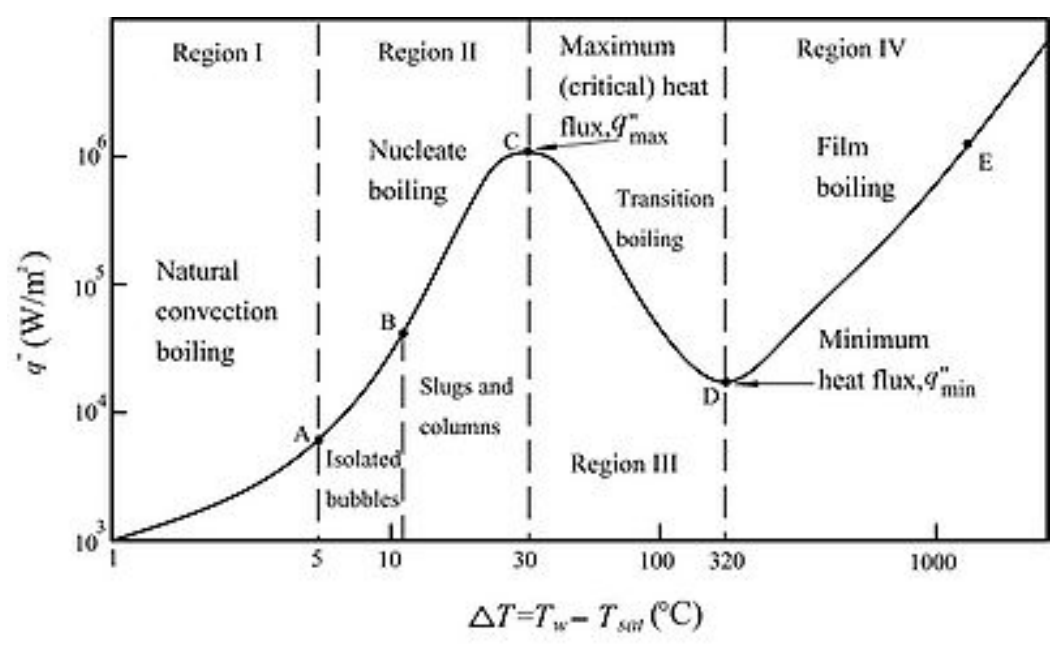

Figure 1: The Boiling Curve. (Resource:

https://www.thermalfluidscentral.org/encyclopedia/index.php/Pool_Boiling_Regimes)

In the experiments conducted in this thesis, the focus was mostly on nucleate boiling below CHF.

\section{Related Work}

Boiling heat transfer has been studied in many industrial applications and has a history spanning more than five decades. But unfortunately, studies of boiling heat transfer in microgravity conditions are very scarce and sometimes present contradictory results. It is also important to note that most research pertaining to boiling in microgravity environments has thus far been either of a qualitative nature using photographic studies with some wall heat flux/wall temperature measurements, or else analytical work, or numerical simulations. Some of these experiments on pool boiling in microgravity have focused on 
varying parameters such as pressure, heat transfer, flow pattern, electrostatic forces and CHF, though some studies have concentrated on more than one of these parameters. In this section, we present a cross section of the literature on pool boiling under microgravity conditions.

Several experiments have been carried out on boiling under microgravity and Earth-like gravitational conditions, and it was found the heat transfer mechanisms to be similar in both cases. In experiments conducted by Straub in 2001, boiling curves from both wires and flat plates at saturated and sub cooled conditions were obtained for three types of liquids, and the boiling curves generated from the results indicated that gravity has little effect on the overall heat transfer, although a large increase in the bubble departure radius was observed. They concluded that the buoyancy effects are replaced by surface tension effects in low-g, so the overall level of heat transfer remains about the same (Straub, 2001). It has also been proven that the boiling heat transfer systems have the advantage of better heat transfer coefficients in the cooling loops, which reduces the total weight of the system (Gabriel, 2007). In order to design such systems for microgravity, it is necessary to predict the heat transfer characteristics of individual bubbles.

In some studies, external fields such as gravity and electrostatic are applied to control the vapor bubble in gaining an understanding of a more multi-physics approach. It was observed that a reduction in gravity increases bubble size, while the application of an electric field decreases the size and increases the bubble detachment frequency. Thus it was determined the addition of an appropriate electric field widens the nucleate boiling region and the heat transfer coefficient becomes almost insensitive to gravity (Di Marco et al., 2011; Di Marco and Grassi 2009; Carrica et al. 1997).

In 1995 Oka et al. studied pool boiling of organics and water in microgravity environments. They found that the bubbles of the boiling organics coalesced along the surface and were comparable to those obtained under normal Earth gravity conditions. But for water, the coalescence of bubbles occurred in the direction normal to the heaters by the suction of smaller newer bubbles into larger bubbles, and boiling heat transfer deteriorated throughout the boiling regime compared to Earth gravity. These differences in bubble behavior were attributed to differences in surface tension and wettability characteristics of the liquids. They also observed a reduction in the heat transfer for the nucleate boiling regime using water as the working fluid, however, for the organic liquids, the deterioration in the heat transfer coefficient due to the microgravity reduction was notable only for high heat fluxes. For these fluids, they noted a reduction 
of approximately $40 \%$ in the CHF when compared with Earth gravity results (Kubota et al., 2011; Oka et al., 1995).

Madhavan et al., 1997 conducted experiments on boiling behavior under a wide range of heat fluxes and sub-coolings. They revealed an eventual dry out of the surface under high heat fluxes at saturated conditions, but steady nucleate boiling at the same heat flux when the liquid sub cooling was increased to $22{ }^{\circ} \mathrm{C}$. When steady nucleate boiling was observed, a very large bubble above the surface acted as a reservoir for numerous smaller bubbles growing on the heater surface, and this large bubble was thought to maintain its size due to a balance between condensation at the top of the bubble and coalescence with the smaller bubbles at its base. Enhancements in the heat transfer of up to $32 \%$ were observed in microgravity compared to Earth gravity. All these observations were attributed to Marangoni convection effects, the tendency for heat and mass to travel to areas of higher surface tension within a liquid. It caused large vapor bubbles to be impelled toward the heater surface and small bubbles to migrate to the heater surface. Thus, boiling heat transfer was enhanced relative to Earth gravity at lower heat fluxes, but the CHF was reduced significantly in microgravity. Increased sub cooling was associated with an increase in heat transfer level. However, CHF appeared to decrease significantly in microgravity (Madhavan et al. 1997).

In experiments conducted in a $490 \mathrm{~m}$ free-drop shaft at the Japan Microgravity Center, Wakayama (1999) reported the magnetic transport of bubbles with a radius of about $0.2 \mathrm{~cm}$ under microgravity in pure water and glycerol-water mixture, and of smaller size bubbles with radii ranging from $0.05-0.075 \mathrm{~cm}$ in fluid of various viscosity (1-69 cp). Magnetic control of the bubbles made it possible to observe the collision and fusion of two bubbles precisely in the above mentioned study.

Kim et al. (2001), studied the effects of gravity on highly sub-cooled pool boiling for Earth gravity and microgravity saturated conditions. Steady state boiling was observed in microgravity and lowg under sub-cooled conditions. A large primary bubble was observed to form on the surface due to coalescence, and this primary bubble moved over the surface coalescing with smaller satellite bubbles. Boiling curves were similar for different gravity conditions at lower wall superheats. However, heat fluxes for high-g were greater at higher superheats, due to the growth of the primary bubble on the surface in low-g. Also, the Earth gravity heat fluxes were slightly lower than the high-g heat fluxes at all superheats. Low heat transfer was associated with the large primary bubble in low-g, and large amounts of heat transfer with the satellite bubbles. The heat flux associated with the satellite bubbles was similar to 
the heat fluxes obtained at Earth and low-g levels, indicating little effect of gravity on the small-scale boiling (Kim et al. 2001).

Kuhlman et al (2005) had designed a previous experiment to drive away the vapor bubbles that remain on the heater surfaces, by injecting air bubbles to simulate the vapor bubbles formed due to boiling at a single nucleation site of a paramagnetic liquid. The air bubbles at the base of the tank were displaced from the magnet surface, because the Kelvin force attracted the paramagnetic liquid to the magnet surface. In that experiment, for one of the parabolas, air bubbles in the control (no-magnet) tank were much larger in microgravity $(1 \mathrm{~cm}$ diameter, versus $2-4 \mathrm{~mm}$ ) and generally never detached from the bottom of the tank. The average bubble velocity for the magnet tank was $43 \mathrm{~mm} / \mathrm{s}$, although there was considerable scatter in these results. The Kelvin force on a bubble adjacent to the magnet surface for this parabola was computed to be approximately $20 \mu \mathrm{N}$, which was judged to be in reasonably good agreement with the computed drag force for these bubbles of $30 \mu \mathrm{N}$. For another parabola, similar to the previous parabola the bubbles in the magnet tank were smaller in diameter (3-6 mm diameter, versus $1 \mathrm{~cm}$ for the control in the no-magnet case). Bubble velocities ranged form about $100 \mathrm{~mm} / \mathrm{s}$ to nearly zero for the bubbles that were repelled from the magnet; this velocity range is similar to that observed for the earlier parabola.

In a study conducted aboard the Chinese recoverable satellite, pool boiling heat transfer on a plain plate in different pressure and sub cooling has been studied. In this study, it was shown that the nucleate boiling curve will be left-shifted with the increase of sub cooling for the same pressure and vice versa, which is analogous to the results obtained under the Earth gravity conditions. However, it was also shown that the CHF in microgravity is no more than $40 \%$ of that under Earth gravity conditions (J. F. Zhao et al. 2007). In another study by the same group, pool boiling on thin wires, plates, and micro-pin-finned surfaces, was studied. It was found that the bubble dynamics in microgravity has a distinct difference from that in normal gravity, and the heat transfer characteristics depended upon the bubble dynamics, In addition, lateral motions of bubbles were observed before their departure in microgravity (J. F. Zhao 2011). 


\section{Chapter 2: Methodology}

This chapter describes the process involving the designing of the experiment, running the experiment on the Zero-gravity flight, how the data was collected during the short time interval where microgravity was experienced during the flight, the image processing techniques applied on the images of the bubbles captured during the flight, and finally the analysis on the data that was done to obtain the final results.

\section{Designing the Experiment}

The experimental apparatus had two identical cylindrical tanks shown in Figure 2. At the bottom of each tank, an electric heating pad is attached to the inner bottom surface. These almost identical heating pads (electric heaters) were rated at $100 \mathrm{~W}$. The tanks are dimensionally identical and the only difference between them was that one tank had a 5000 Gauss permanent magnet (nominal) kept below the heater as shown in Figure 2 below. The tank without the magnet serves as non-magnet control tank, for comparison purposes.

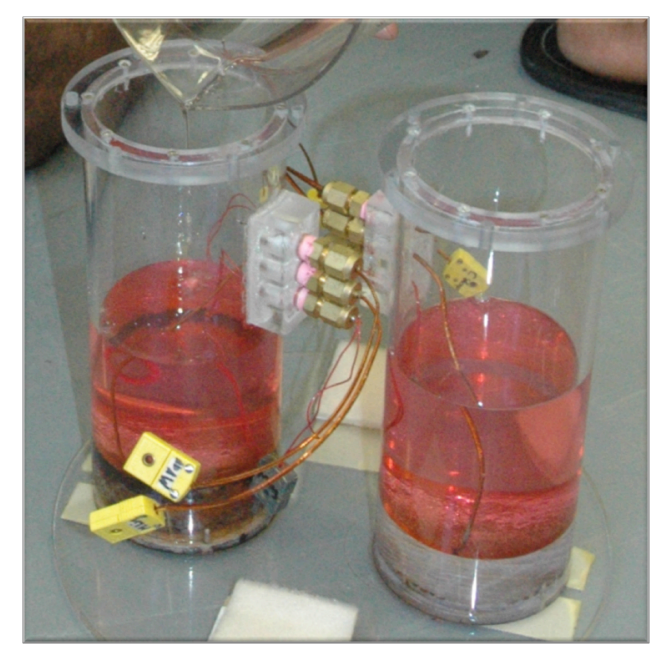

Figure 2: Two Identical Inner Tanks

One large containment tank was used to contain any spills or leaks from these two identical tanks. The liquid solution in the two identical tanks was heated to the same temperature simultaneously at the same time, by also keeping the two tanks at the same pressure at the same time. The liquid solution used for the boiling purpose was $\mathrm{MnCl}_{2}$ with a molarity of 4.5. The $\mathrm{MnCl}_{2}$ was dissolved in distilled water to 
create the 4.5-molarity solution, and was used in both tanks in order to make sure that each tank had the same liquid with the same molarity.

The power for the heater pad was set using a Variac, which goes from zero to $100 \mathrm{~W}$. Each heater pad gets the same amount of power from the Variac at the same time since they were wired in parallel to the variac. By varying the Variac, the amount of heat that gets produced by heaters can be changed as desired during the experiment. During the experiment, the Variac settings were kept between the ranges of $40 \%-70 \%$. Boiling process was conducted and observed under different Variac settings within that range. Below a setting of $40 \%$, little or no boiling was observed, while above $70 \%$ the liquid would foam out the top of the two tanks in microgravity.

The external tank that held the two inner tanks along with other experimental components was mounted on an aluminum frame which was custom built by welding. This frame was $102 \mathrm{~cm}$ (40 inches) long and $40.6 \mathrm{~cm}$ (16 inches) wide and $66 \mathrm{~cm}$ (26 inches) tall. The frame was constructed using an aluminum tube that has the square cross section of $3.81 \mathrm{~cm}(1.5$ inches $)$ and $0.32 \mathrm{~cm}$ wall thickness. At the bottom of the frame, an $0.64 \mathrm{~cm}$ (0.25 inches) aluminum plate was welded and attached to create the bottom surface or the floor of the frame. This bottom floor was used to attach the outer containment, vacuum pump and one video camera. A separate shelf within the frame was created to mount the Variac. That shelf was $35.6 \mathrm{~cm}$ (14 inches) long and $40.6 \mathrm{~cm}$ (16 inches) wide in dimension and was held $33 \mathrm{~cm}$ (13 inches) above from the bottom floor of the aluminum frame.

The two inner tanks were made with transparent acrylic tubing with a thin wall. The dimensions of the tanks are given in Table 1. The outer containment tank is shown in Figure 3, while a close-up view of the two inner tanks is shown in Figure 4, under boiling conditions.

Table 1: Dimensions of the Tank

\begin{tabular}{|c|c|}
\hline Inner Diameter & $7.30 \mathrm{~cm}(2.857$ inches $)$ \\
\hline Outer Diameter & $8.26 \mathrm{~cm}(3.25$ inches $)$ \\
\hline Height & $21.6 \mathrm{~cm}(8.5$ inches $)$ \\
\hline Flange Diameter & $9.85 \mathrm{~cm}(3.875$ inches $)$ \\
\hline Flange Thickness & $0.64 \mathrm{~cm}(0.25$ inches $)$ \\
\hline
\end{tabular}




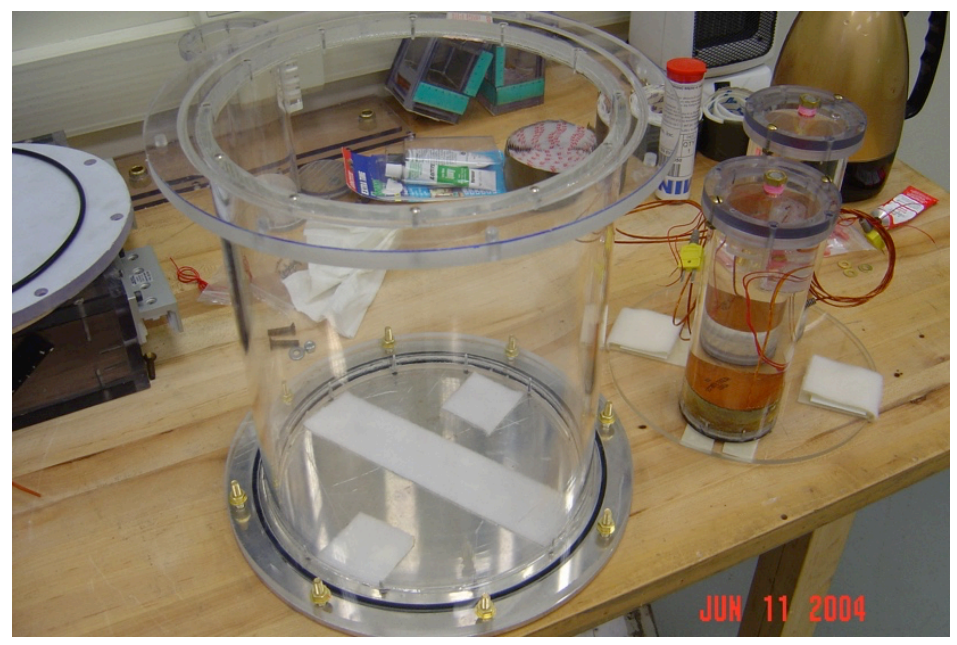

Figure 3: The Outer Containment Tank

The following Figure 4 shows the components of the inner tanks, in operation at a relatively low heat flux condition in Earth gravity conditions.

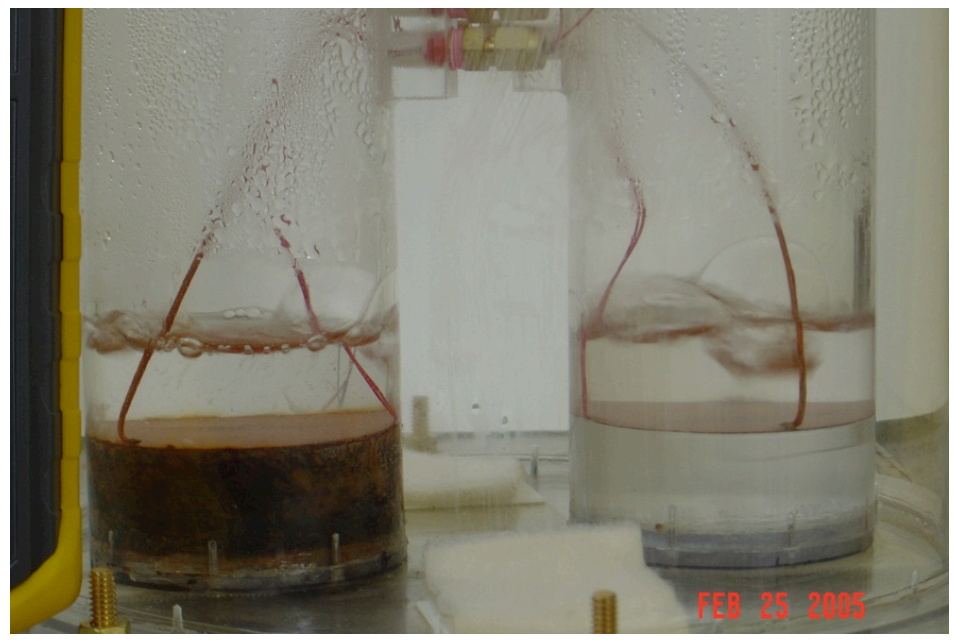

Figure 4: Close up Image of the Inner Tanks

As can be seen in the Figure 3, each inner tank has a vent on the top of the tank's lid. The heater power leads and thermocouples were penetrated inside to the tank by attaching rectangular Plexiglas bulkhead as shown in the Figure 5 below, and as seen in Figure 2. 


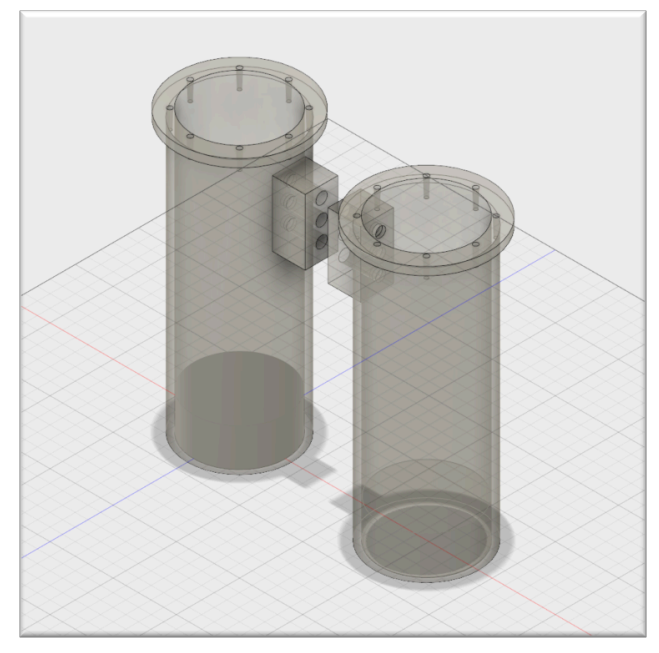

Figure 5: CAD Drawings of the Inner Tank

Waterproof epoxy was used to seal the tank components completely in a way that liquid inside cannot leak outside of the tank. Before assembling the bottom part of the tank to the cylindrical part, a circular heating pad was glued to the bottom circular part of the tank.

Four Type-K thermocouples were used to determine the temperature of the boiling liquid. Each inner tank had two thermocouples; one was kept at the bottom, touching the surface of the heating pad in order to determine the temperature at the heater surface and the other one was kept at a height of $12.7 \mathrm{~cm}$ (5 inches) from the bottom surface. Those thermocouples were read by using two Omega HH5010K 4channel thermocouple readers that were mounted next to the tank as shown in Figure 6 below.

The 5000 Gauss Dexter Magnetic Technologies Neo38 permanent magnet, with a cylindrical shape was custom made with the diameter of $7.3 \mathrm{~cm}$ (2.87 inches) and $2.54 \mathrm{~cm}$ (1 inch) thickness as the height. This was used as the magnet that was kept below one of the tanks, and that tank was identified as the magnet-tank. 


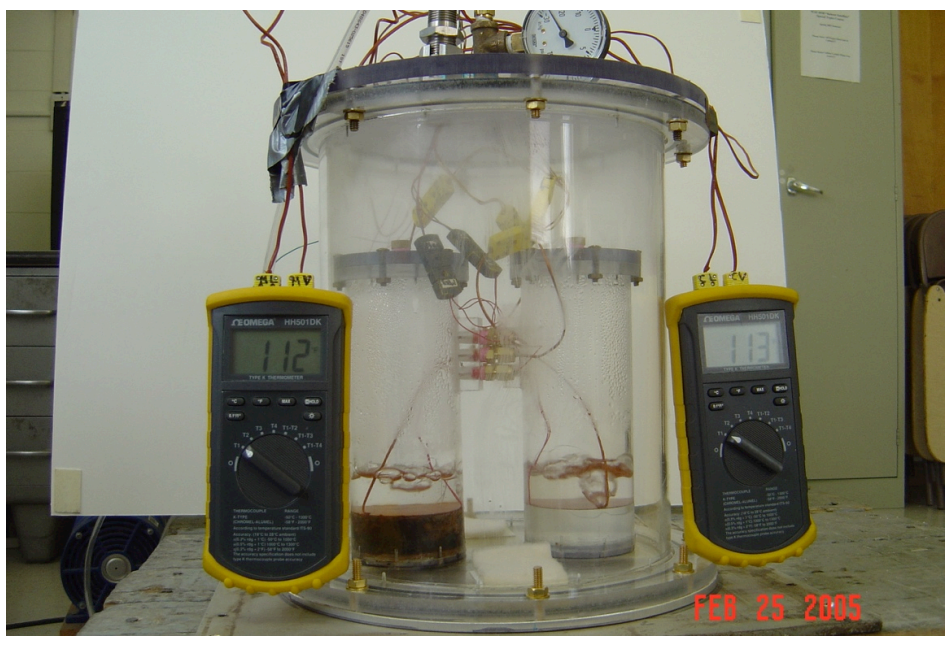

Figure 6: Tanks with Omega HH5010K 4-Channel Thermocouple Readers

The following Table 2 shows the strength of the magnetic field along the vertical axis of the tank, measured from the magnet surface, and Figure 7 plots these values on a logarithmic scale.

Table 2: Magnetic Strength Along the Vertical Axis

\begin{tabular}{|c|c|}
\hline Axial Distance (m) & Magnetic Field Strength (Gauss) \\
\hline 0 & 3642.22 \\
\hline 0.01 & 1489.33 \\
\hline 0.02 & 881.22 \\
\hline 0.03 & 576.11 \\
\hline 0.04 & 396.11 \\
\hline 0.05 & 276.33 \\
\hline 0.06 & 203.11 \\
\hline 0.07 & 151.33 \\
\hline 0.08 & 111.22 \\
\hline 0.09 & 90.11 \\
\hline 0.1 & 71.55 \\
\hline
\end{tabular}




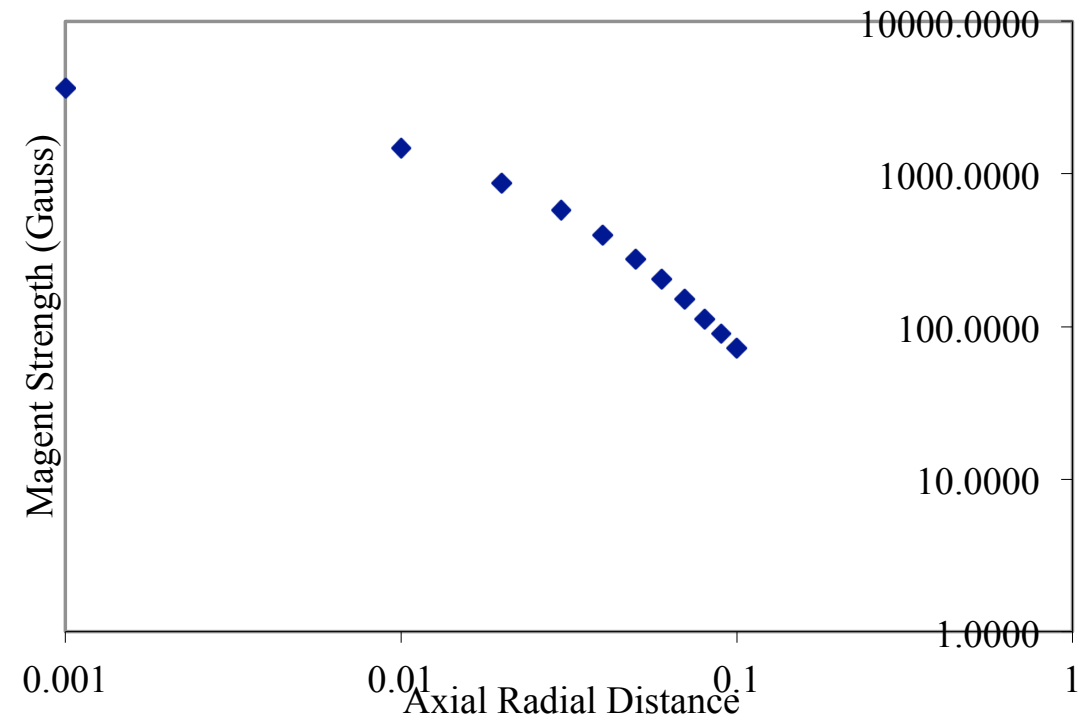

Figure 7: Visual Representation of the Strength of the Magnet Field Along the Axial Distance of the Magnet Tank

The other tank was kept as the control tank (non-magnet-tank) by keeping a blank Plexiglas cylinder that had the same dimensions of the magnet and was kept at the bottom of the tank.

Identical, Minco SSG3R100L12E 100W Kapton heaters (heating pad) with the diameter of 7.24 $\mathrm{cm}$ (2.85 inches) were glued to the bottom component (base) of the each tank as shown in Figure 8. During the laboratory testing prior to the flight, the obtained measurements values at the room temperature resistance of the four heaters were almost identical.

In order to have a homogenous bubble generation throughout the surface of the heater, sand grains were applied over both heater surfaces to provide nucleation sites as shown in Figure 9. The sand grains with a diameter ranging from 100 microns to 120 microns were carefully filtered, by using industrial standard filters as shown in the Figure 10. 


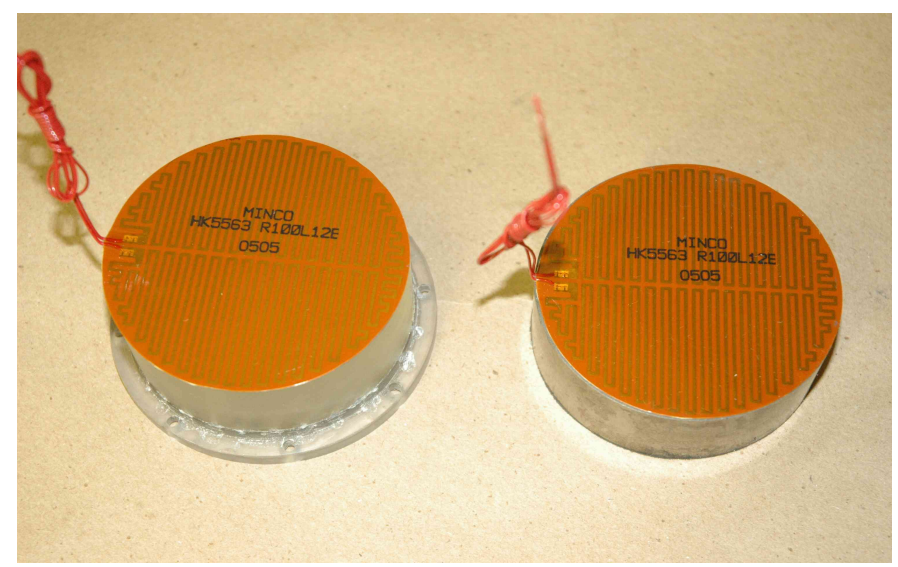

Figure 8: $100 \mathrm{~W}$ Heater on the Base of Magnet Tank and Non-Magnet Tank

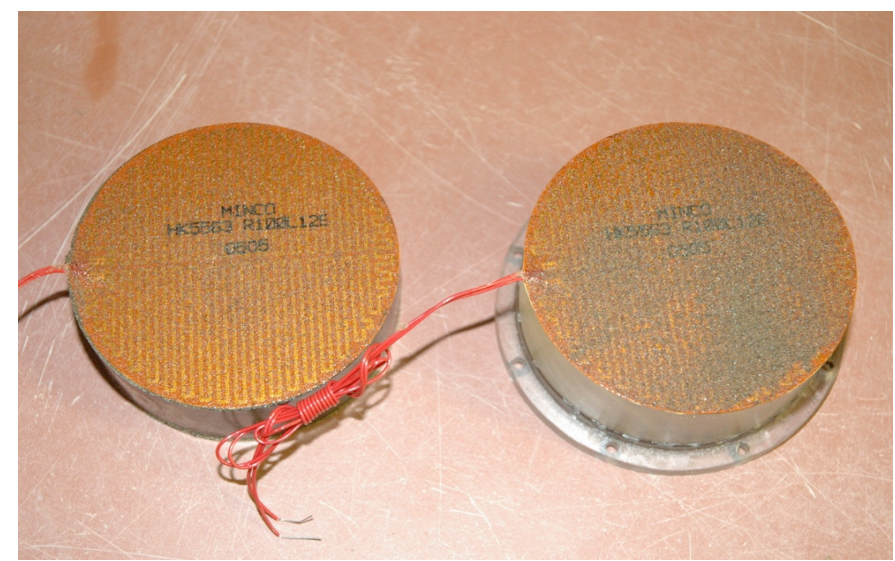

Figure 9: Heater Surface After the Sand Grain Applied

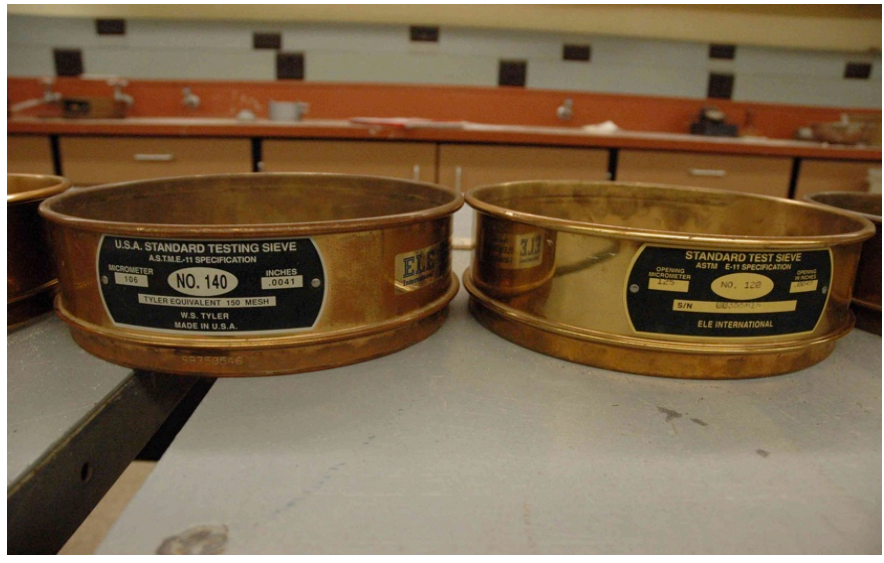

Figure 10: Industrial Standard Sand Filters 100 - 120 microns 
As a safety requirement, the entire frame was wrapped with fireproof padding material before the flight. Final setup of the experiment mounted to the frame and installed in the Zero-G Corporation 8-727 aircraft is shown in Figure 11.

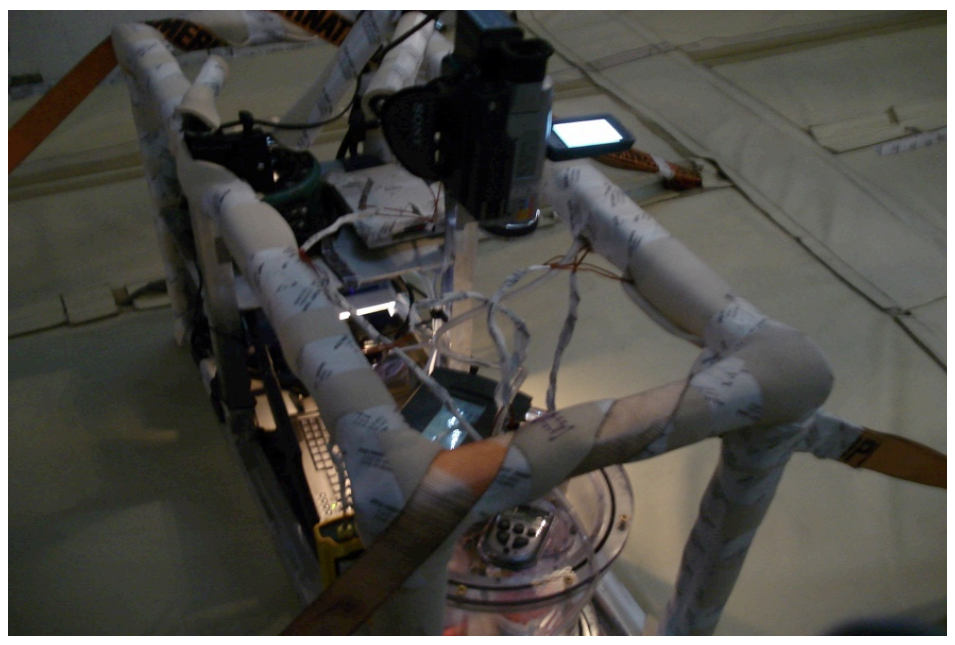

Figure 11: Final Experiment Setup Before the Flight

An anti-fog film was pasted on the inner wall of the outer containment tank in the viewing regions to keep it clear as much as possible to obtain a clear visual view of the running experiment to capture on video clips. The two JVC Everi GZ-MG-21u model digital cameras were used to record boiling phenomena during the flight. One camera was zoomed and pointed in the direction where the thermocouple readers were mounted in a manner to capture their values on the video clips. This camera, pointing at thermocouple readers was mounted to the bottom of the frame and it was named as "FrontView-Camera". The video clip obtained by this camera was named as "Front-View" during the post analysis work. Since this camera was mounted to the bottom of the frame and during the flight, available light inside the aircraft cabin was significantly low and dark. Due to that, it was difficult to use the video clip that was obtained from this camera to observe the boiling behavior.

The second camera, known as the "Back-View" camera was mounted using a camera tripod to the aircraft cabin floor. This camera was pointed and zoomed in the direction where it captured a view of both the inner tanks. The video that was captured from this camera has been used to observe the bubble behavior during the boiling process throughout the flight primarily because this view was unobstructed and had better lighting. The still images captured from the "Back-View-Camera" were processed and enhanced using image processing software during the post analysis work. Due to the unexpected low 
light inside the flight cabin, these images were less clear than was anticipated at the time of the experiment design and set up prior to the flight.

\section{Running the Experiment}

\section{Zero-gravity Flight}

We flew in the Boeing 727 aircraft from Zero-G Corporation in a parabolic path, in which the microgravity conditions lasted for a short period of 20-30 seconds for each parabola. The reduced gravity environment inside the aircraft cabin was experienced during the parabolic path maneuvers over the course of two hours in flight. Those parabolic path maneuvers are termed as "Parabolas". There were 40 parabolas for each of the two flights, which generated reduced gravity conditions. Some parabolas reached the microgravity conditions and some were able to reach some level of reduced gravity conditions based on the pilot's ability. The average time of reduced gravity condition during the parabolas was 22.5 seconds.

\section{Experiment Procedure}

The experiment was kept continuously running throughout the flight. The length of the reduced gravity time was obtained from the verbal information that was recorded with the video when the flight cabin coordinator's announcements at the beginning and end of the parabolic maneuvers. The flight cabin coordinator's verbal announcements of the start and end times were compared with the changes in boiling pattern and its duration.

Parabolas 34 and 35, with the durations of 30 seconds and 26 seconds, respectively, gave the steadiest microgravity conditions compared to other parabolas. This is due to the higher length in duration and also the value of the Variac settings, which was held at 52\%. During the parabolas 34 and 35, the aircraft cabin pressure was documented as 29.97 In $\mathrm{Hg}$ (101.49 kPA). The cabin pressure and the cabin temperature were verbally announced along with the readings displayed for the four channels of data coming from the thermocouples. Transcription of the audio information that was recorded during the 
flight provided additional information, which was essential for the analysis work. The Variac settings with respect to parabolas provided key information as to what power was supplied to heaters. This was obtained from the carefully documented and recorded verbal information from the cabin crewmembers who assisted and the two researchers who conducted the experiment.

There was a vacuum pump connected to the outer containment tank as part of the experiment to conduct boiling at various levels of pressure. Prior to the flight, flight cabin crew noticed an effect due to the use of the vacuum pump on the aircraft circuit breakers. The vacuum pump was causing the circuit breakers to go off and due to that fact; the vacuum pump was unable to be used during the flight. As a result of that, liquid was boiled at the temperature that corresponding to the cabin pressure of the aircraft, somewhat above $100^{\circ} \mathrm{C}$, due to the 4.5 molal solution of $\mathrm{MnCl}_{2}$.

\section{Data Collection}

The method of data collection from the experiment was predominantly video observation of the boiling process. Along with the visual information gathered from video cameras, verbal information spoken by the flyers was also recorded. Aircraft cabin pressure, temperature and readings from the thermocouples channels were spoken out by the flyers.

The total length of the video clip of "Back View" was 63 minutes. The original Back View video was recorded in "mpg" format. During the analysis, the video was converted to the higher quality MP4 format without changing the original aspect ratio or the frame dimensions. Later, the new enhanced Back View MP4 format video was used to extract the video segments relevant to the microgravity or reduced

gravity periods. These extracted video segments were then used to obtain image frames. The "Adapter"2 software was used to extract still image frames from the pool boiling video clips. Those sequences of images were used during the image processing to obtain bubble information.

${ }^{2}$ https://www.macroplant.com/adapter/ 


\section{Parallax and False Contouring Issues}

Since the bubble data has been obtained from the images, which were extracted from the captured videos clips of the experiment, researchers are aware of the possible existence of the parallax related issues. The parallax errors in instruments (parallax error in videography and photography), and parallax errors in sight (parallax in optical sight) could possibly affect the distance measurements. In general, it is advisable to pay attention to these possible uncertainties in measurements. Similarly, due to false contouring, it is possible to misjudge or misidentifying the actual or the absolute boundary of the bubble by the naked eye. Therefore, it is possible to introduce errors in the hand-drawn line segments that are slightly off from absolute margin of the bubble boundary. This could add some uncertainty to the values obtained from data obtained from the images. Thus, the parallax issues and false contouring issues with respect to image analysis and camera angle(s) may have slightly affected the accuracy of the obtained results of this experiment.

The Figure 12 shown below demonstrates the effects of parallax. The height distance of 40.8 millimeters of the display panel was measured using a vernier caliper as shown in the figure. This measurement activity was captured on an image and four different lines on four different locations were drawn to demonstrate the height distance of the display panel. The line 1 was used to set the measurement and declared as 40.8 millimeters. The lines 2, 3 and 4 were drawn representing the display panel's height as their lengths and the length values of those lines were calculated. The measured distances of the lines are shown in Table 3. Even though, all four lines represents the same distance visually, their actual measured distance values are different from each other. Ideally, these four lines must provide the same distance of 40.8 millimeters on the image, but due to the parallax, different values at different locations on the images were witnessed. The measured distances of the lines are shown in the Table 3 , and there is approximately $10 \%$ difference between the computed lengths of line 1 and line 4 .

Table 3: Difference Between the drawn line segments

\begin{tabular}{|c|c|}
\hline Lines & Measured Length \\
\hline line 1 & $40.8 \mathrm{~mm}$ \\
\hline line 2 & $38.4 \mathrm{~mm}$ \\
\hline line 3 & $37.6 \mathrm{~mm}$ \\
\hline line 4 & $37.0 \mathrm{~mm}$ \\
\hline
\end{tabular}




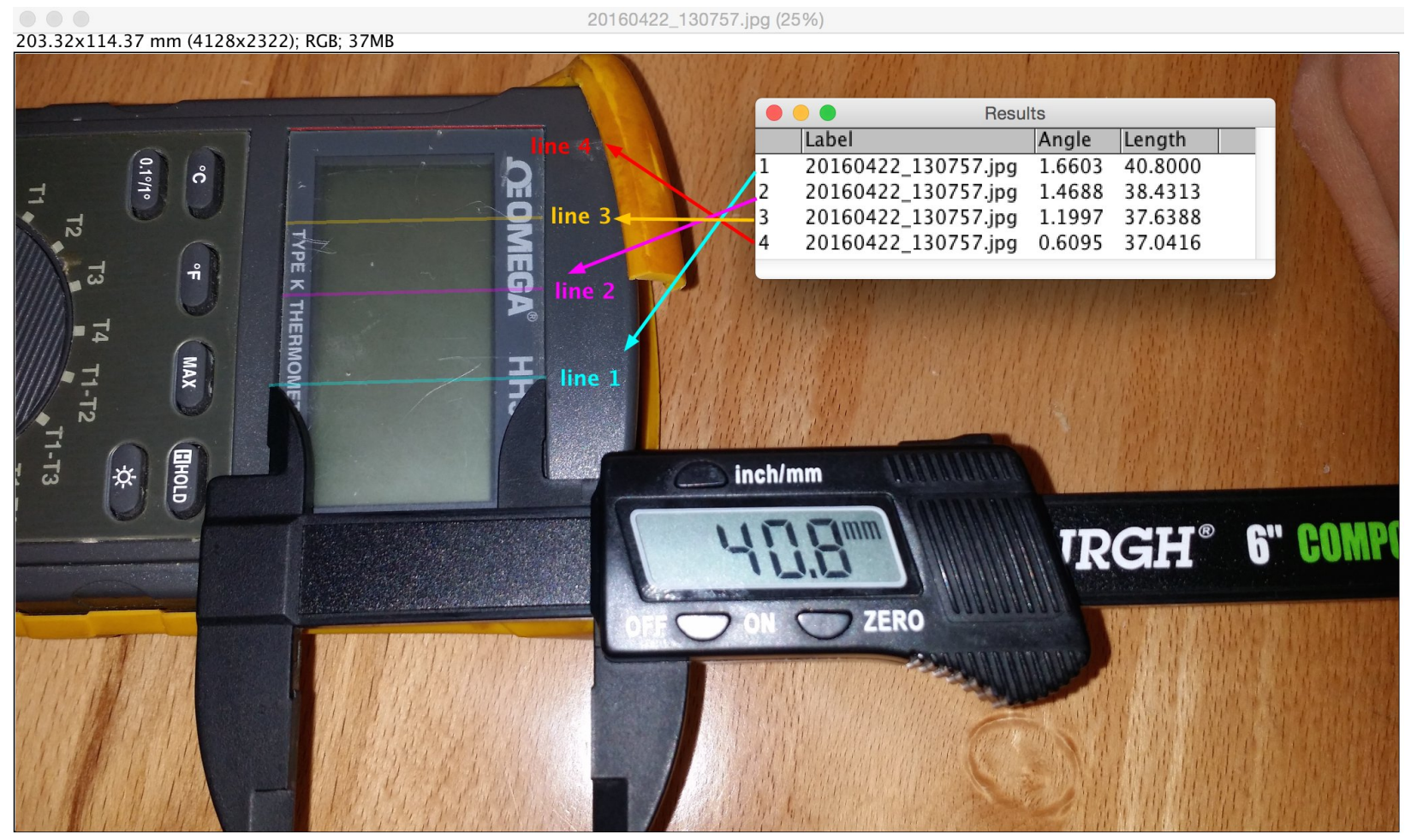

Figure 12: Measuring and Comparing the Known Line Distance on an Image Over Multiple Locations to Demonstrate the Issues of Parallax

\section{Data Analysis}

A variety of techniques were used in analyzing the data collected from the experiments described above. In this section we explain the image processing techniques used in processing the images from extracting the bubble images from the image sequences, obtaining the bubble specific data from the images such as the area, and the calculations performed on the bubble data.

\section{Selecting the Best Possible Data}

The Parabolas 34 and 35, which lasted for 30 seconds and 25 seconds respectively, with the Variac settings at $52 \%$, had the best pool boiling information out of all parabolas for the entire 
experimental flight time. Even though the other two parabolas, 36 and 37 were at the same Variac settings, the boiling time periods in microgravity conditions were short. Thus, due their higher information richness parabolas 34 and 35 were chosen for analysis using several image processing techniques as outlined below.

\section{Extracting Image Sequences from the Video}

As mentioned before, the software that was used to extract image frames from the video clip is Adapter. This software allows the user to choose the required frame rate number based on the number of frames per seconds (fps) that the video has recorded. The videos taken during the flight were recorded at 30 fps. Using the Adapter software, it is possible to obtain frames at any time interval, i.e. create an image sequence with 5 frames per second, 3 frames per second, etc. With trial and error, it was discovered that when every $10^{\text {th }}$ frame is taken (i.e. 3 frames every second), it yields enough data without too much redundancy and also showed the bubble progression clearly. Thus, the time between the selected frames is one third of a second. Using this method, a sequence of images of the bubbles was generated from the videos for analysis with Image $^{3}$, and image processing and analysis library written in Java, as explained below.

\section{Image Processing Techniques Using ImageJ}

The primary image processing library used for analyzing the videos and images obtained from the zero gravity flight is Image $J^{3}$ (Ferreira and Rasband, 2012). ImageJ is a public image processing and, analysis tool inspired by the National Institutes of Health. The program runs, either as an online applet or as a downloadable application, on any computer with a Java 1.5 or later virtual machine. In the analysis done for this thesis, the ImageJ Mac OSX application was used. Using the tool, it was possible to display, edit, analyze, process, save and print 8-bit, 16-bit and 32-bit images of the bubbles, in a variety of image formats including TIFF, GIF, JPEG, BMP and 'raw'. Since ImageJ supports 'stacks' (and hyper stacks), a series of images can share a single window. To stack a set of images, they must all be the same width, height, and bit depth. Stacks can animate the images at speeds from one frame every 10 seconds to over 1000 frames per second. Many operations, such as selecting, filtering, thresholding, and contrast

\footnotetext{
${ }^{3}$ http://imagej.nih.gov/ij/
} 
enhancement can be applied to all slices in a stack. Stacks are typically used to display and analyze images that are related to each other in some way. Since the bubbles have both time (temporal) and space (spatial) relationships to each other, we found it easier to analyze and precisely measure the same regions the bubble images using stacks.

In addition, the program is multithreaded, so time-consuming operations such as image file reading can be performed in parallel with other operations. The primary usage of ImageJ in this thesis was in calculating area and pixel value statistics of certain selections that include the bubbles. Additionally, Image J was used to measure distances and angles, as well as to create density histograms and line profile plots. Some of the other standard image processing functions such as zoom, contrast manipulation, sharpening, smoothing, edge detection and median filtering, and more advanced functions including geometric transformations such as scaling, rotation and flips were also used to fine-tune the results. We will use the image as shown in Figure 13 and explain the types of transformations, selections and calculations done on that image, and on all other related images in an image sequence or stack.

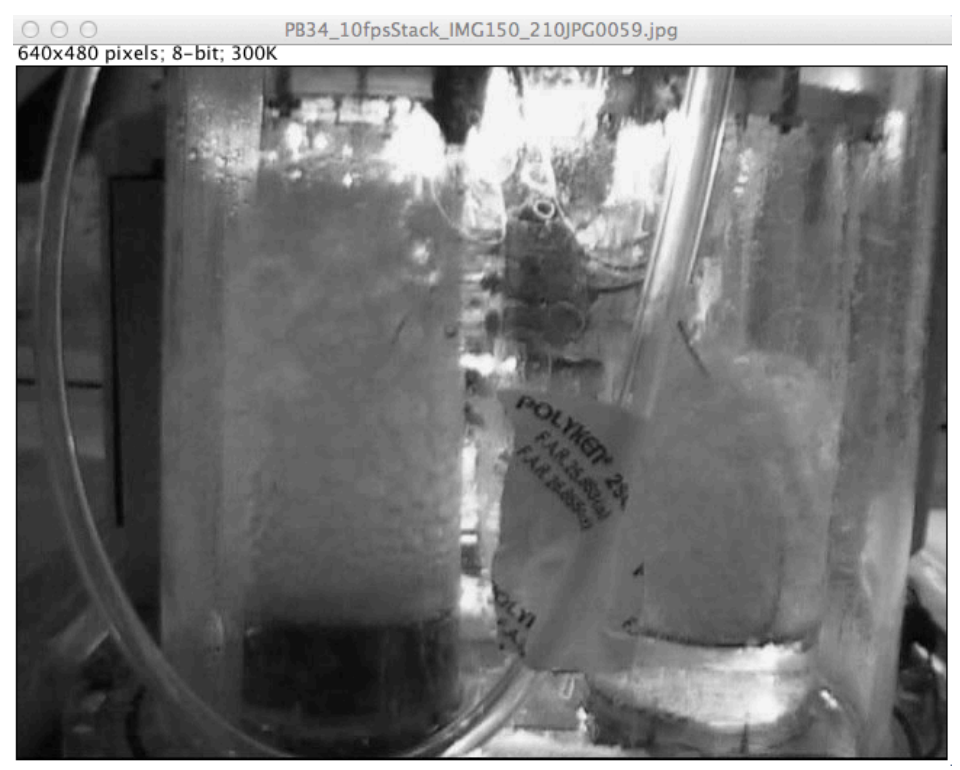

Figure 13: Still Frame of the Tanks With the Bubbles 


\section{Basic Image Processing Techniques:}

The sequence of images that were obtained from the video can be opened as a "stack" in ImageJ. In order to open as a stack of images, all the images must have same type and size for each image that are saved inside the folder. Each image has some metadata, such as the image width, height, size, bits per pixel as shown in Figure 14.

The major and minor axis and the coordinates of a selected point can be obtained using the "cross-hair" as shown in Figure 15. It was necessary to change the brightness and contrast of many images to increase the visibility of the bubble perimeter. Figure 16 shows how this was done.

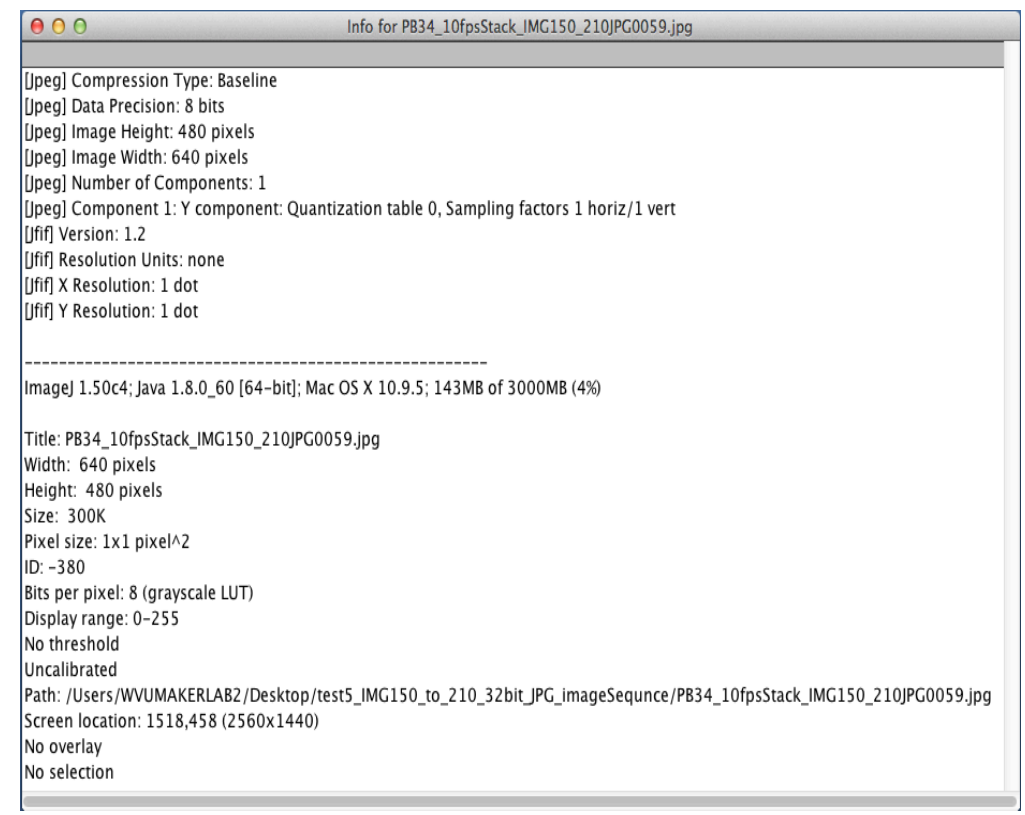

Figure 14: Metadata of the Selected Image 


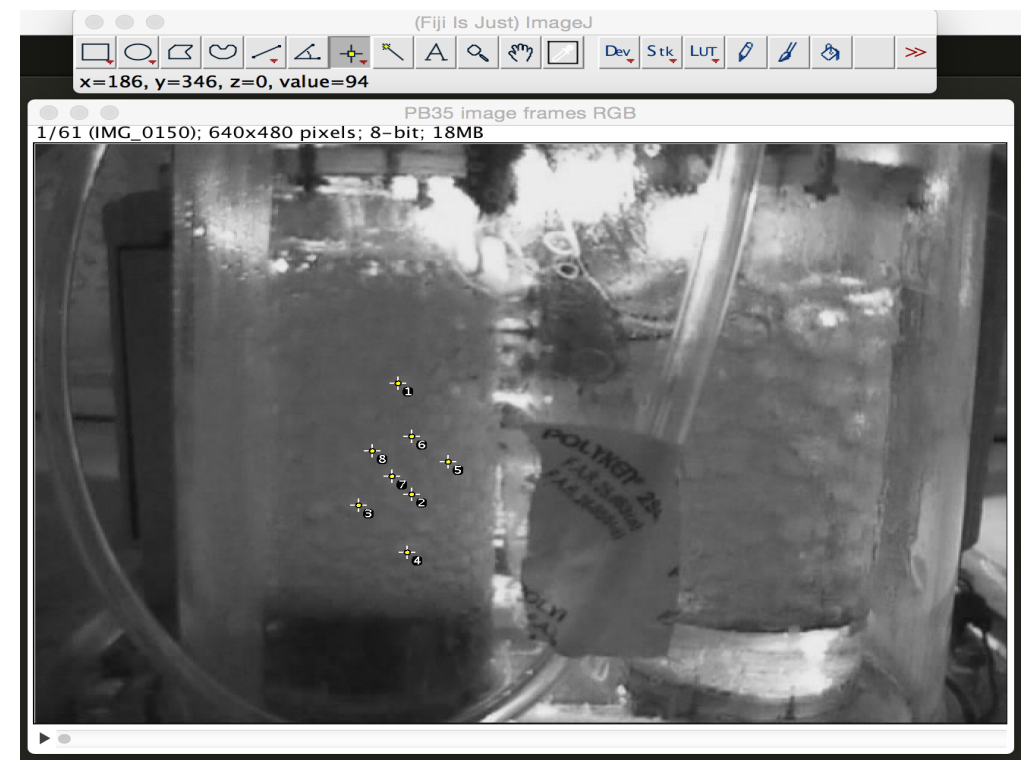

Figure 15: Obtaining the co-ordinates of bubbles along the Major and Minor Axes

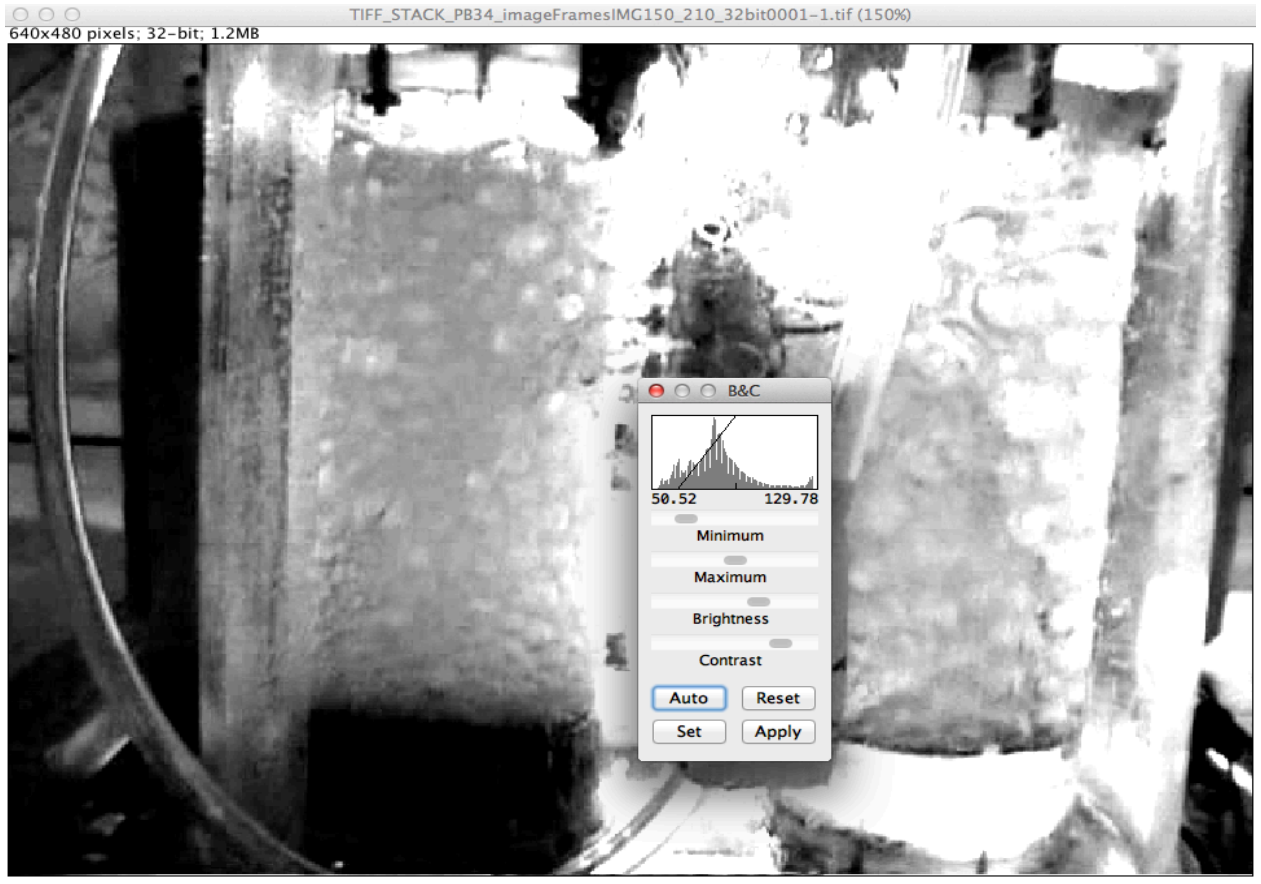

Figure 16: Changing the Brightness and Contrast 


\section{Selecting a Bubble Area:}

Figure 17 shows some of the information worthy areas on the image frame. Based on the selection of the area, the size of the image gets narrow, and unworthy areas or sections that have higher or unbalanced illumination which effects the overall pixel intensities can be avoided. This gives a bit of an advantage to have a relatively balanced light intensity over the image. After determining the chosen bubble on the image frame, the area or the region of that bubble where it is located on the image frame can be selected and saved as a selected area. Figure 18 shows the original image and the selected area image. The yellow color selection rectangle's pixel height and width is equivalent to the new "Selected Area" image's pixel height and width. After selecting the area, a new selected area image can be quickly generated by the following process of Image $>$ Duplicate command on ImageJ menu.
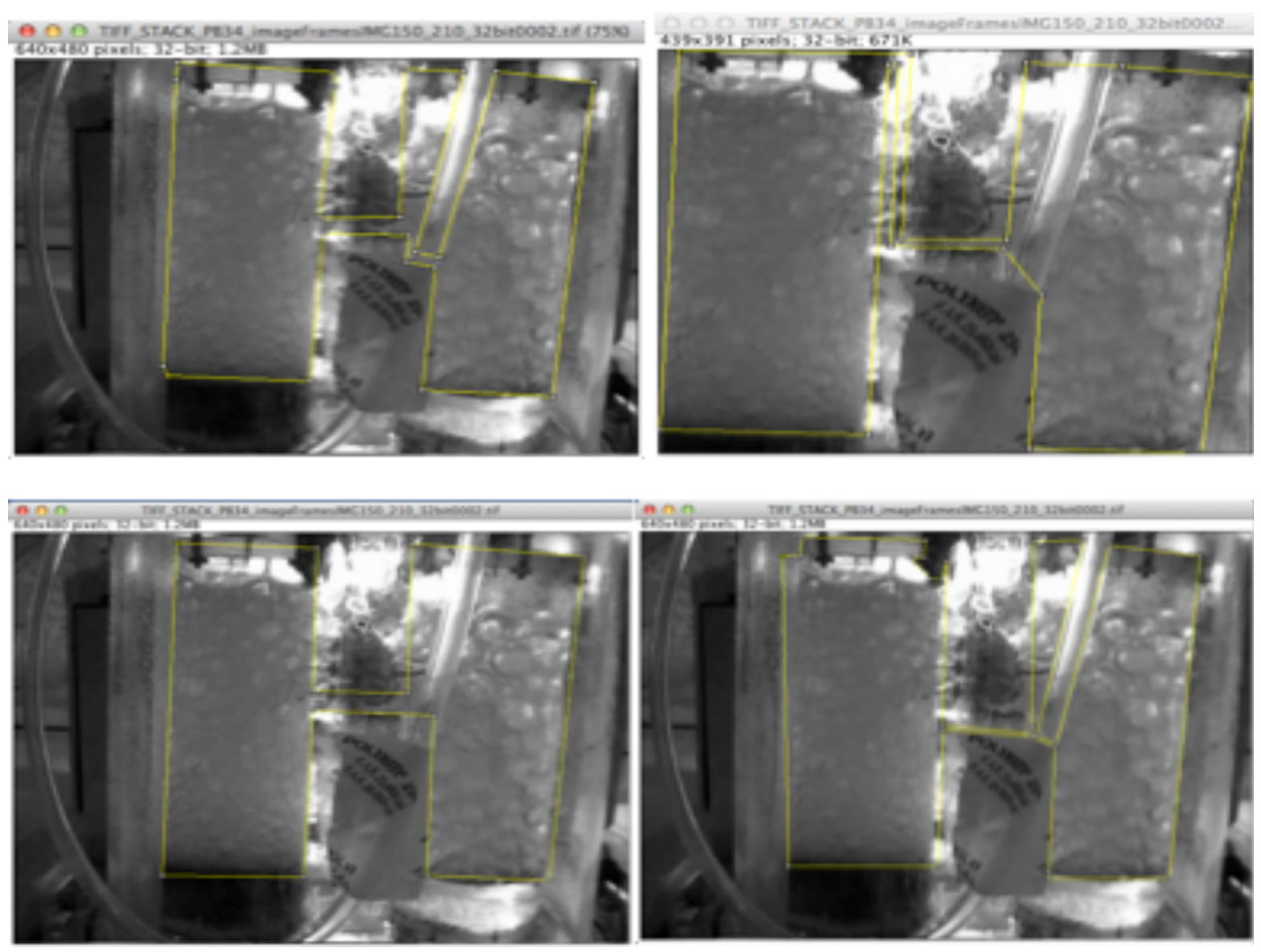

Figure 17: Information Worthy Areas of Some Image Sequences 


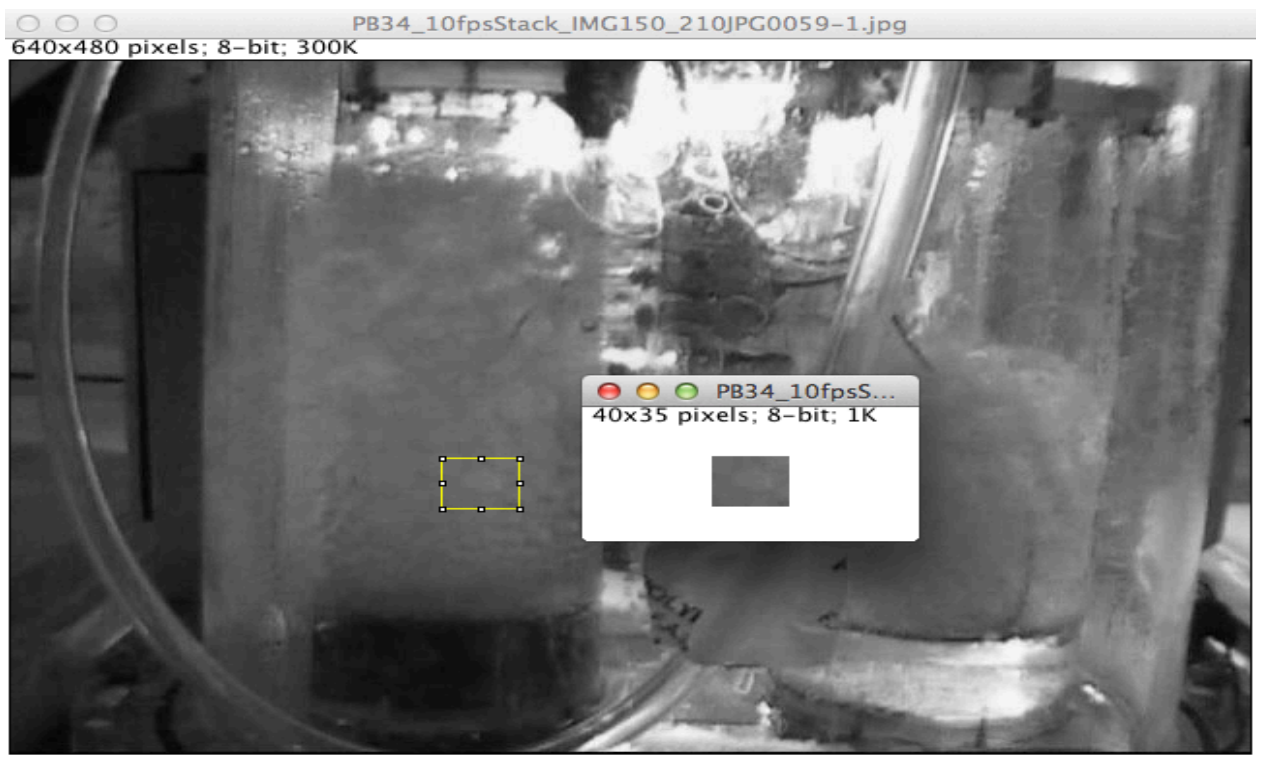

Figure 18: The Original Image and a Selected Bubble From the Image 


\section{Selecting a Region Of Interest (ROI):}

Using mouse clicks it is possible to "draw" on the image to select an ROI that will be the bubble perimeter as shown in Figure 19. Figure 20 displays the chosen set of preferred measurements checkboxes have checked to obtain the data of the hand selected ROI shown in Figure 19. This is prior to conducting the ellipse fitting, and notice that the "Fit Ellipse" checkbox is not chosen at the moment. When an ROI is set to fit an ellipse, ImageJ replaces an area selection with the best fit ellipse. The ellipse will have the same area, orientation and centroid as the original selection. The same fitting algorithm is used to measure the major and minor axis lengths and angle when Fit Ellipse is selected in Analyze $\rightarrow$ Set Measurements.

The obtained data of the ROI will be compared with the data obtained after the ellipse fitting process. This comparison will show the available variations due to force fitting of an ellipse to the hand selected ROI. This usually has the same moments (more specifically, 1st and 2nd moments) as the bubble area that was hand drawn (using the mask, i.e., the area; the gray scale value is ignored). This method usually results in an ellipse with a slightly different area than the bubble. Therefore, as a last step, in ImageJ, the ellipse is scaled to have the same area as the bubble. ImageJ conducts the calculations on the selected set of measurements with the keyboard command of "CMD+M", after which it shows the results obtained before and after an Ellipse fitting. The second row of this table represents the calculated data (obtained results) after force fitting an ellipse to the hand selected (hand drawn) ROI. 


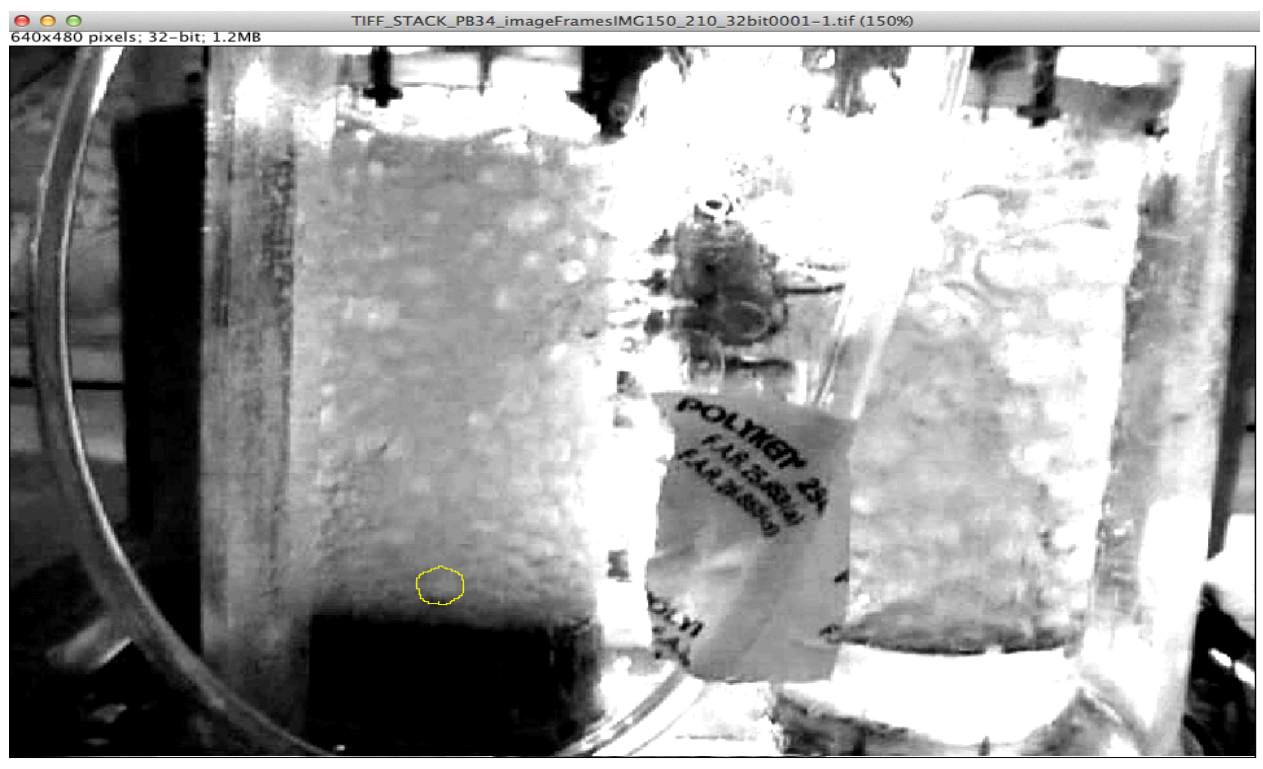

Figure 19: Hand-drawn ROI of a Bubble

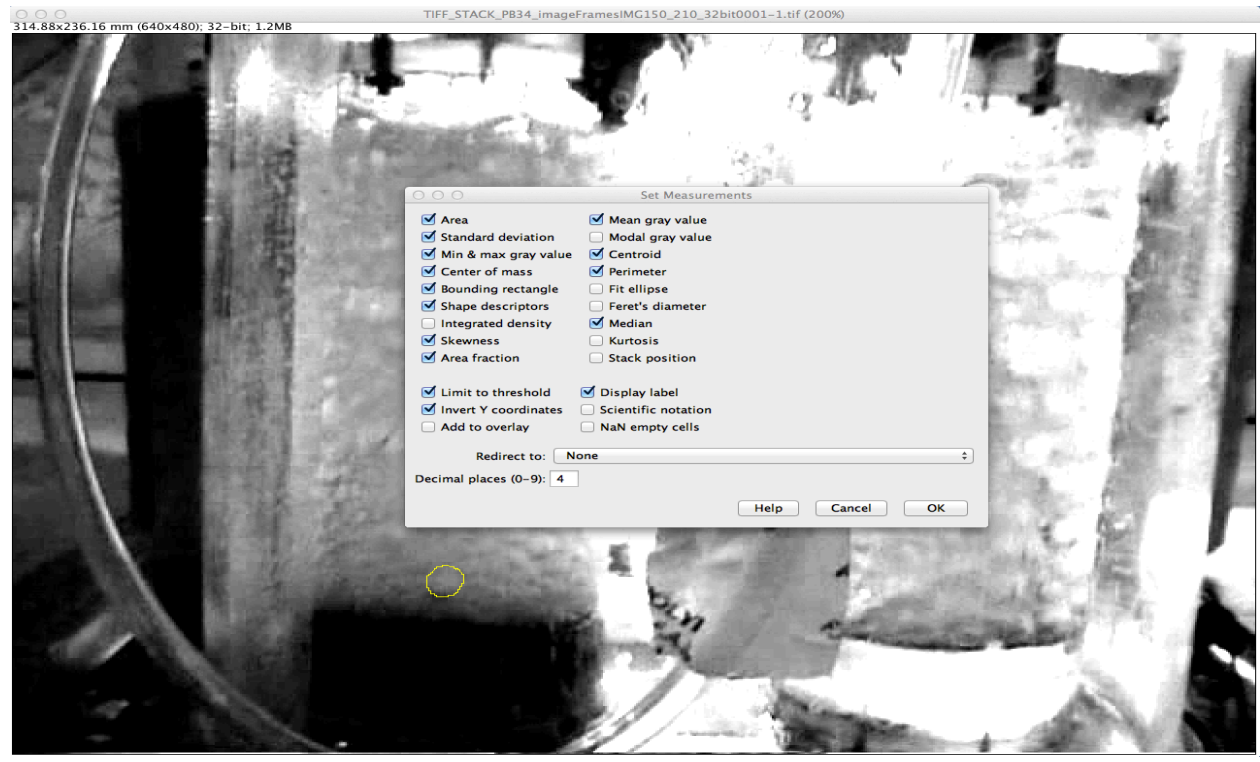

Figure 20: ROI Property Selection 


\section{Spatial Calibration}

In order to gather measurements from an image using real values (for example in $\mathrm{mm}$ ), a single image can be calibrated against known values. As can be seen in Figure 21, the tank's flange was measured using a Vernier caliper. The measured width was $9.02 \mathrm{~mm}$. Then, in an image of the tank, ImageJ's line selection tool was used to draw across the flange. With the selection line present, "Set Scale" function was selected from the Analyze menu, which brings up the dialog box as can be seen in Figure 22. The distance is pixels and the pixel aspect ration was already filled in, and the known distance was given as 9.02 with the unit of length being $\mathrm{mm}$. As can be seen in the Figure 22, ImageJ calculates the scale to be 2.0322 pixels $/ \mathrm{mm}$, and applies the same ratio on all of the images that are being analyzed. This spatial calibration was especially useful when obtaining the distances along the semi major and semi minor axes in mm as described in Chapter 3.

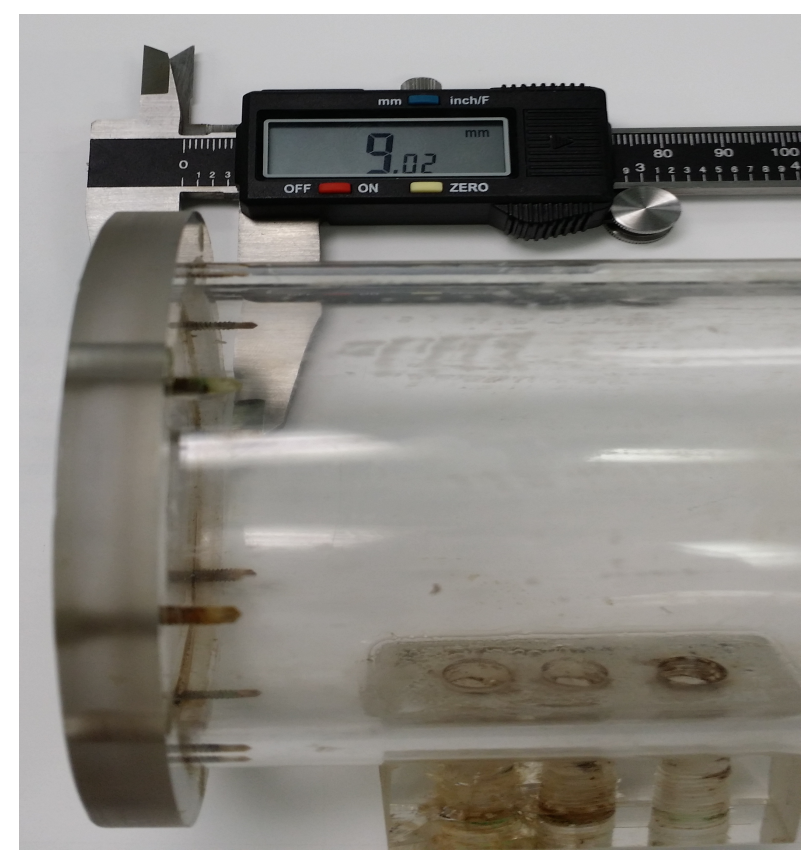

Figure 21: Tank's Flange measured using a Vernier Caliper 


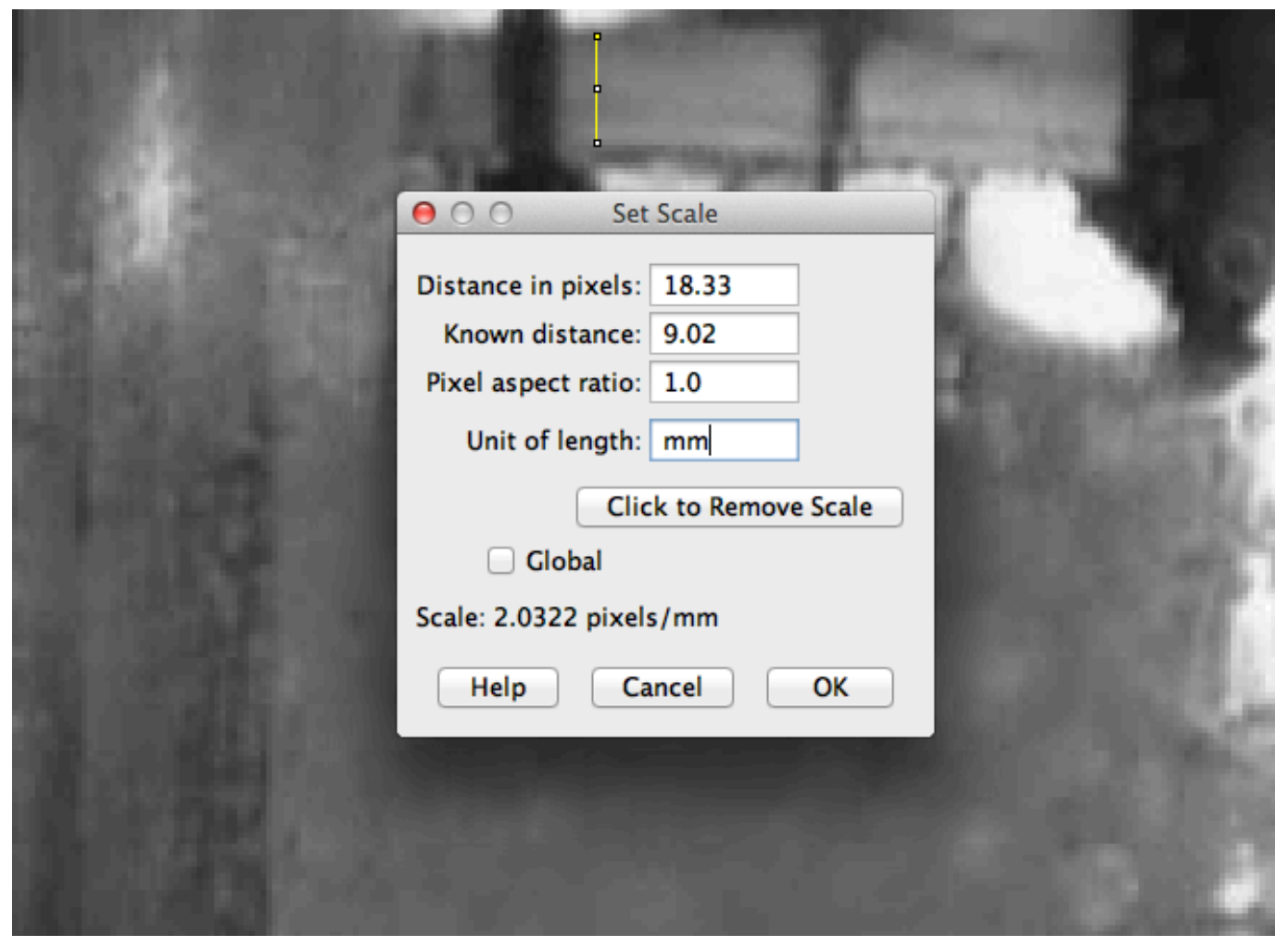

Figure 22: Set Scale Function Used to calibrate the Tank's Flange

\section{Image Processing Techniques Using Matlab}

As described in the Appendix, the Matlab Image Processing Toolbox was initially used to obtain the bubble data. The image processing toolbox from Matlab was very flexible and has many built-in functions that were useful. However, a lot of coding was required to obtain many of the data points needed for this thesis. Matlab was essentially lacking a nice full-featured user interface (UI) for experimenting with images, and only supported a code editor and command window. Comparatively ImageJ that we later used included a very convenient UI, had the various functions needed for image processing in its menu, and plenty of tutorials online that helped learn various image processing techniques useful for obtaining the data for the thesis. ImageJ enabled us to apply the same transformation to images visually, which was not possible in Matlab. Furthermore, ImageJ is free and open source, while Matlab is not. 


\section{Chapter 3: Results}

In this chapter we summarize the gravitational impact on the bubble behavior during pool boiling by analyzing the bubble characteristics: namely, bubble size, velocity, shape and trajectory, as well as the body forces acting on the bubbles.

\section{Bubble Size Calculation Method}

By comparing the horizontal and vertical radius of the bubble at the bottom of the tank and the top of the tank, we were able to recognize the major difference in shape. At the bottom of the tank the bubble's vertical radius is comparatively smaller than the horizontal radius. This pattern was seen in different samples obtained from the various parabolas. The distortion in the bubble shapes is probably due to the fact that when the bubble is at the bottom of the tank, it goes through the strongest region of the magnetic field, and as the bubble goes along the tank, the strength of the magnetic field reduces and eventually the vertical component of the radius gets bigger than the horizontal radius. We also observed that after leaving the bottom region, the vertical radii component of the bubble starts increasing. As the bubbles pass the middle region of the tank, the horizontal and vertical components of the radii become closer to each other and bubbles tend to be more circular or spherical in the shape. This was observed in several samples obtained from different parabolas. As the bubbles enter to the top region of the tank, the vertical radii component dominates. When the bubbles reach more towards the upper section of the top region of the tank, again the bubble's horizontal and vertical components of the radius get more close to one value. At the top of the tank, the magnetic field strength is very weak and bubble's shape again demonstrates a circular shape with almost equal horizontal and vertical radius. To verify the above observations, many measurements of the bubbles in different parabolas were taken by analyzing the video frames one by one.

The bubble size was analyzed as the two dimensional area of the bubble appearing on an image frame. First, several bubbles from parabola 34 were chosen for analysis and were tracked from the bottom of the tanks to the top of the tanks as they travelled through the liquid. For nine such sample frames, an outline of the bubble's contour was drawn on the image frame in ImageJ to select the region of interest. 
Then the region of interest was fit to a circle and ellipse and the areas obtained again. The data obtained from this method is plotted in Figure 23.

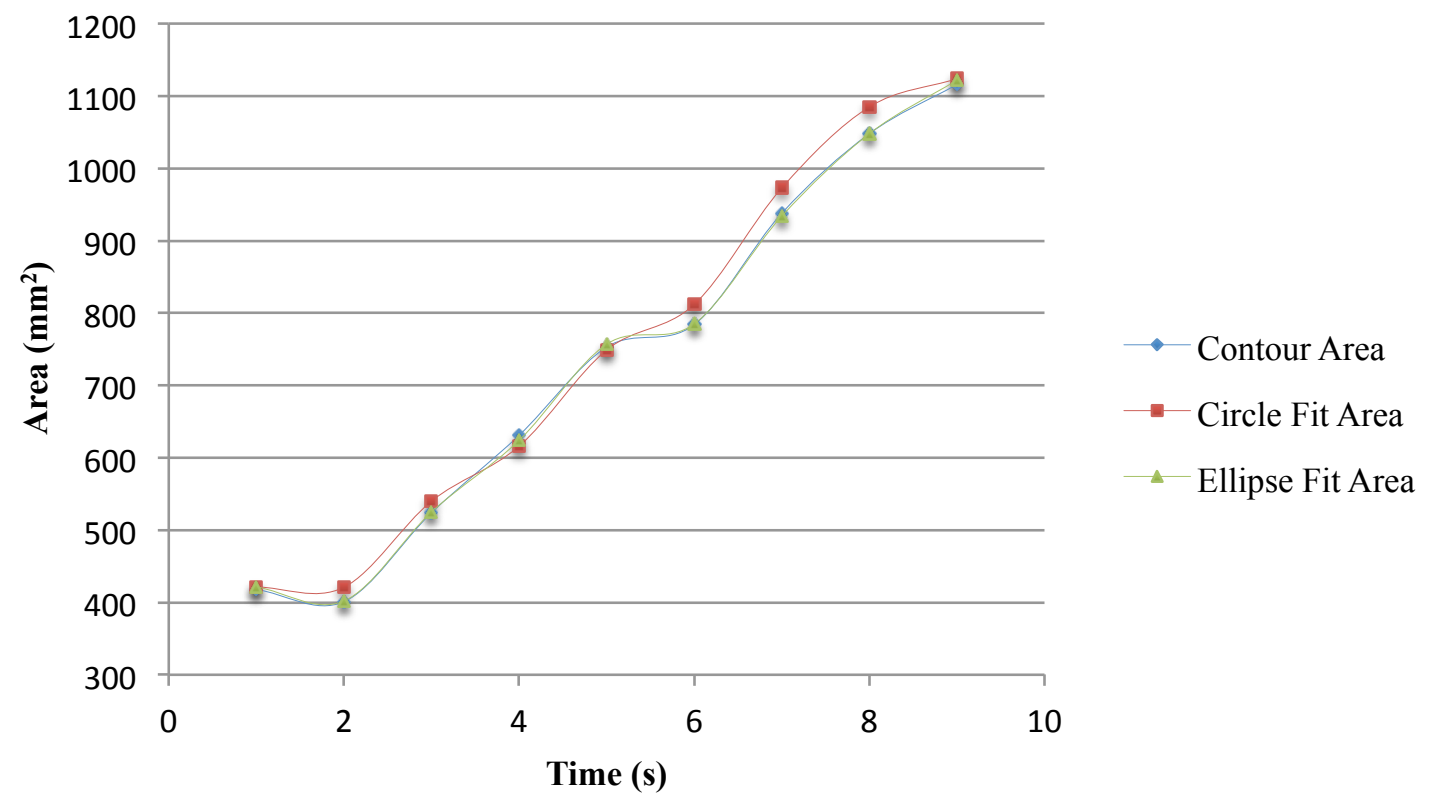

Figure 23: Area Calculation using Region of Interest Method for Bubbles; Parabola 34

The above graph demonstrates that the ellipse fit has similar values to the contour fit, which is the most accurate area we can obtain from the video frames. Since the above process required very tedious image tracing at each frame, the area of the bubbles were calculated using the horizontal and vertical diameters of the bubble. It was observed that many of the bubbles were not exactly circular in shape due to deformation; thus, an extra step was necessary to obtain both the lengths of the semimajor and semiminor axes (half of the ellipse's major and minor axes) of the elliptical bubble. After obtaining the lengths of each axes, the bubble area was calculated considering that the bubble has an ellipse shape by using the following formula, where a and $\mathrm{b}$ refer to the distance along the bubble along the semimajor and the semiminor axes:

\section{Equation 1: Bubble Area as an Ellipse}

$$
A_{\text {ellipse }}=\pi a b
$$


We also manually calculated the area of the bubble as a circle for comparison using the following equation where the radius was taken as the average of the distances along the semimajor and semiminor axes for comparison:

\section{Equation 2: Bubble Area as a Circle}

$$
A_{\text {circle }}=\pi\left(\frac{a+b}{2}\right)^{2}
$$

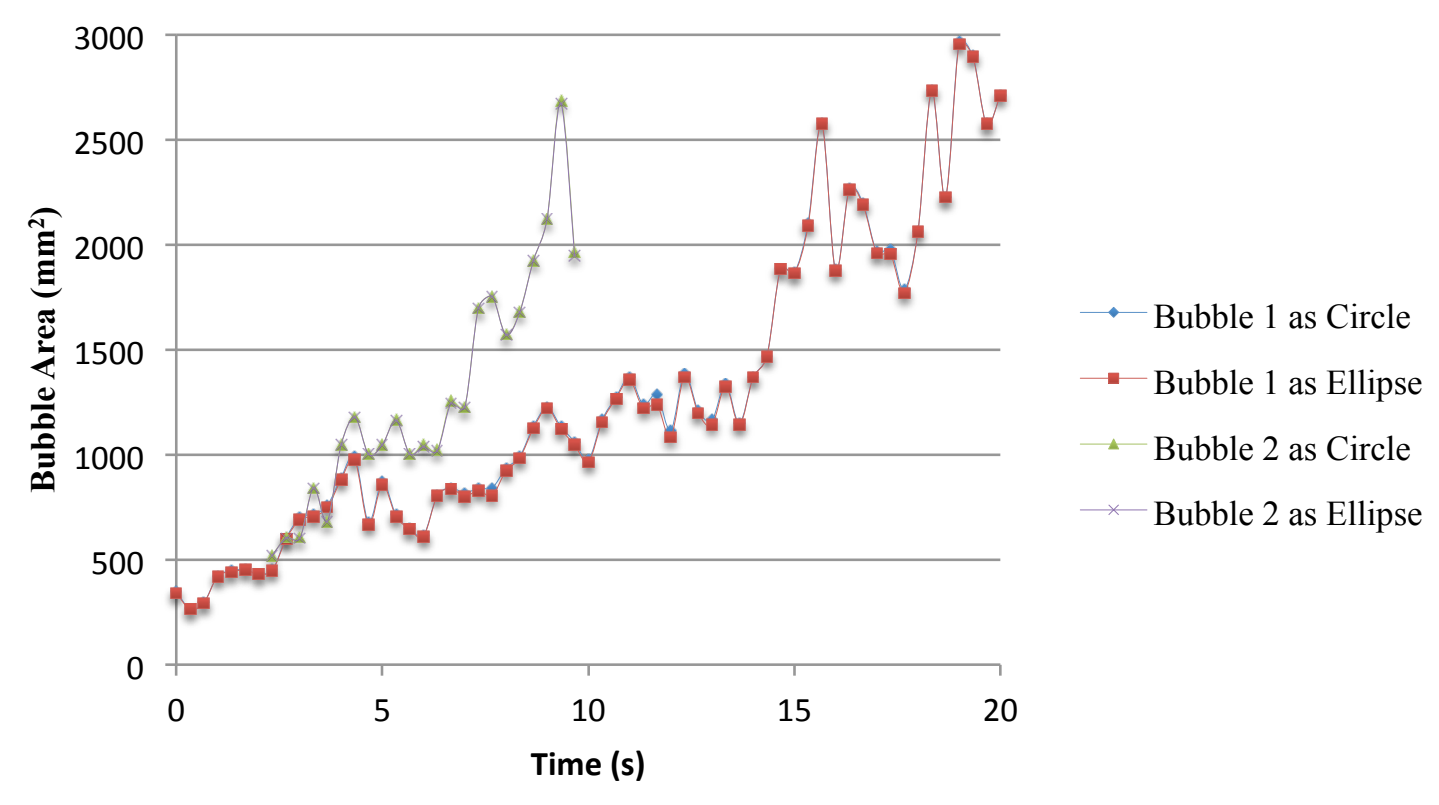

Figure 24: Comparison of Bubble Area Calculated as a Circle and as an Ellipse;

Parabola 34

Figure 24 shows the comparison of bubble area for two selected bubbles in parabola 34. The results indicate only very minor differences in the size. The difference between the two calculation methods by assuming one of the bubble's (Bubble 2) two-dimensional shapes as a circle and as an ellipse is given in Table 4. The value of area difference for the two different calculation techniques gets higher as the bubble moves upwards along the tank, because the bubble size is increased. The difference values are small as the bubble size is small when the bubble is at the bottom region compared to the top region of the tank, thus the circular shaped area value and ellipse shaped area values are close to each other. As the bubble gets bigger, this difference gets higher as well. However, the average percentage difference between the two bubble fitting methods was $0.26 \%$, and did not exceed even a single percent. Therefore, 
the calculation of the area of the bubble by assuming its shape as a circle or an ellipse was assumed not to provide significantly different values.

Table 4: Percentage Difference Between Circle and Ellipse Areas for Bubble 2

\begin{tabular}{|c|c|c|c|}
\hline Frame & Area as a Circle $\left(\mathrm{mm}^{2}\right)$ & Area as an Ellipse $\left(\mathrm{mm}^{2}\right)$ & Percentage Difference \% \\
\hline 1 & 520.98 & 518.57 & 0.46 \\
\hline 2 & 605.05 & 603.82 & 0.20 \\
\hline 3 & 605.05 & 601.07 & 0.66 \\
\hline 4 & 842.73 & 842.29 & 0.05 \\
\hline 5 & 683.77 & 683.57 & 0.03 \\
\hline 6 & 1046.77 & 1045.98 & 0.08 \\
\hline 7 & 1179.80 & 1179.36 & 0.04 \\
\hline 8 & 1004.19 & 1001.79 & 0.24 \\
\hline 9 & 1046.77 & 1046.57 & 0.02 \\
\hline 10 & 1164.63 & 1164.43 & 0.02 \\
\hline 11 & 1004.29 & 1003.85 & 0.04 \\
\hline 12 & 1047.21 & 1038.61 & 0.82 \\
\hline 13 & 1022.72 & 1018.42 & 0.42 \\
\hline 14 & 1256.01 & 1244.17 & 0.94 \\
\hline 15 & 1225.91 & 1224.14 & 0.14 \\
\hline 16 & 1698.91 & 1698.13 & 0.05 \\
\hline 17 & 1754.26 & 1753.82 & 0.03 \\
\hline 18 & 1573.44 & 1571.04 & 0.15 \\
\hline 19 & 1680.69 & 1676.71 & 0.24 \\
\hline 20 & 1925.20 & 1925.00 & 0.01 \\
\hline 21 & 2124.57 & 2124.38 & 0.01 \\
\hline 22 & 2688.91 & 2673.00 & 0.59 \\
\hline \multirow[t]{3}{*}{23} & 1964.29 & 1948.38 & 0.81 \\
\hline & & & Average \\
\hline & & & 0.26 \\
\hline
\end{tabular}

\section{Relationship Between Bubble Size and Time}

Bubble size was observed with respect to the height of the tank, where the size of the bubble at the bottom, middle and the top regions were compared. The bottom region of the tank, which is the closest region to the magnet, has the highest level of magnetic field strength and the top region has the lowest magnetic strength region; see Figure 7. 
In order to analyze the bubble characteristics, the samples with the best data were analyzed, and it was discovered that Parabola 35 had several good bubbles that lasted for at least 25 consecutive frames without disappearing into the background.

Understandably, in order to take the measurements, the bubbles need to be seen clearly. Therefore, when selecting the bubbles, there likely is a bit of a sampling bias. Even though the bubbles are continuously traversing either in the center or much closer to the center from the tank's inner wall or little bit away from center of the tank, only the bubbles somewhat closer to the front facing inner wall were selected as they are the most visible. Due to these selection criteria, there is a possibility that the chosen bubbles might represent slightly skewed results that might reflect some less or slightly different average bubble behavior when compared to a chosen bubble from the exact center of the fluid, which would have been the ideal or the most suitable normal bubble behavior. Since this is commonly applied for all the data samples obtained from the experiment, and the distance from the center of the tank to the inner wall is comparatively smaller in general, it is fairly acceptable to a satisfactory extent to assume the possible difference is negligible.

Using such good bubble data obtained from Parabola 35, Figure 25 shows the increase in bubble sizes during the pool boiling process in the tank with the magnet as time progresses (the frame number is directly proportional to time). Out of the seven bubbles shown, it is evident that some bubbles start boiling early, and disappear quickly into one another (For example bubble 1 and bubble 2, whereas some bubbles increase in size for a much longer duration. Even though a general upward trend is evident, there is a jagged nature in many of the bubbles observed. This can be attributed to coalescence of some bubbles.

The control, the tank without the magnet, as shown in Figure 26 shows a similar trend for the five bubbles chosen. However, in general, the bubbles formed in the non-magnet tank assume larger bubble sizes compared to the bubbles formed in the tank with the magnet. This phenomenon is demonstrated in Figure 27 with the average bubble areas, computed by averaging all of the bubble area results from each of the previous two figures. 


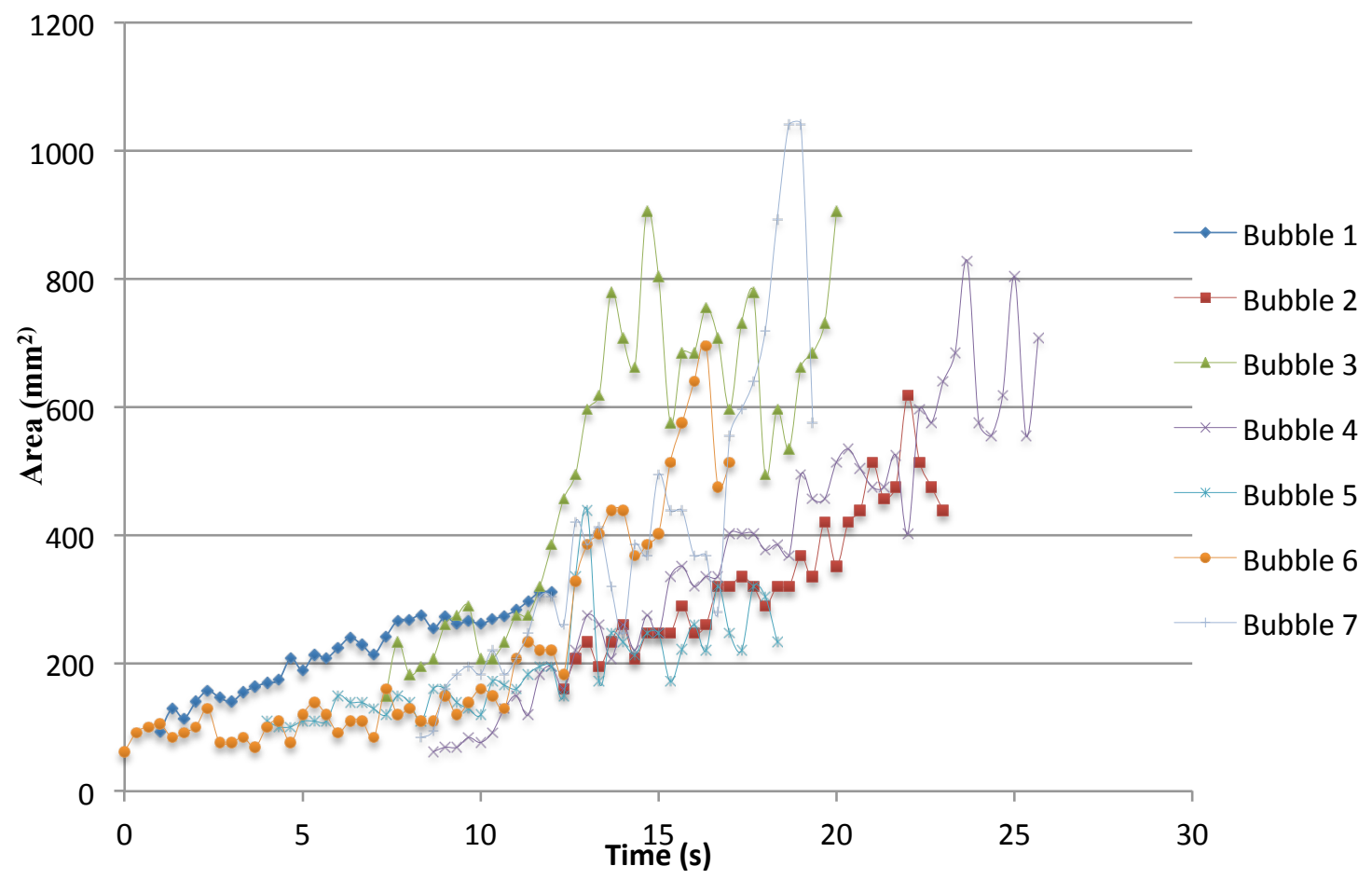

Figure 25: Areas of Bubbles with Respect to Time (Magnet Tank); Parabola 35

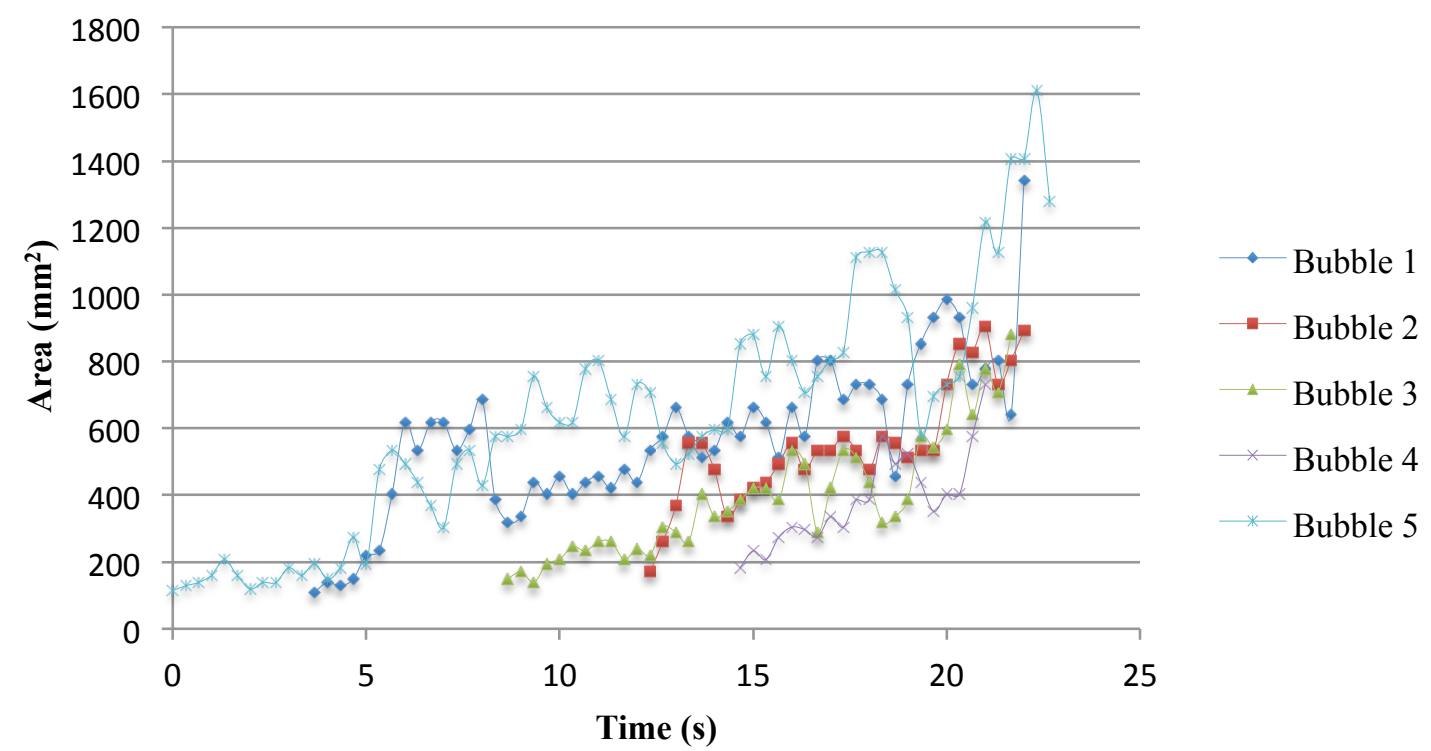

Figure 26: Areas of Bubbles with Respect to Time (Non Magnet Tank); Parabola 35 


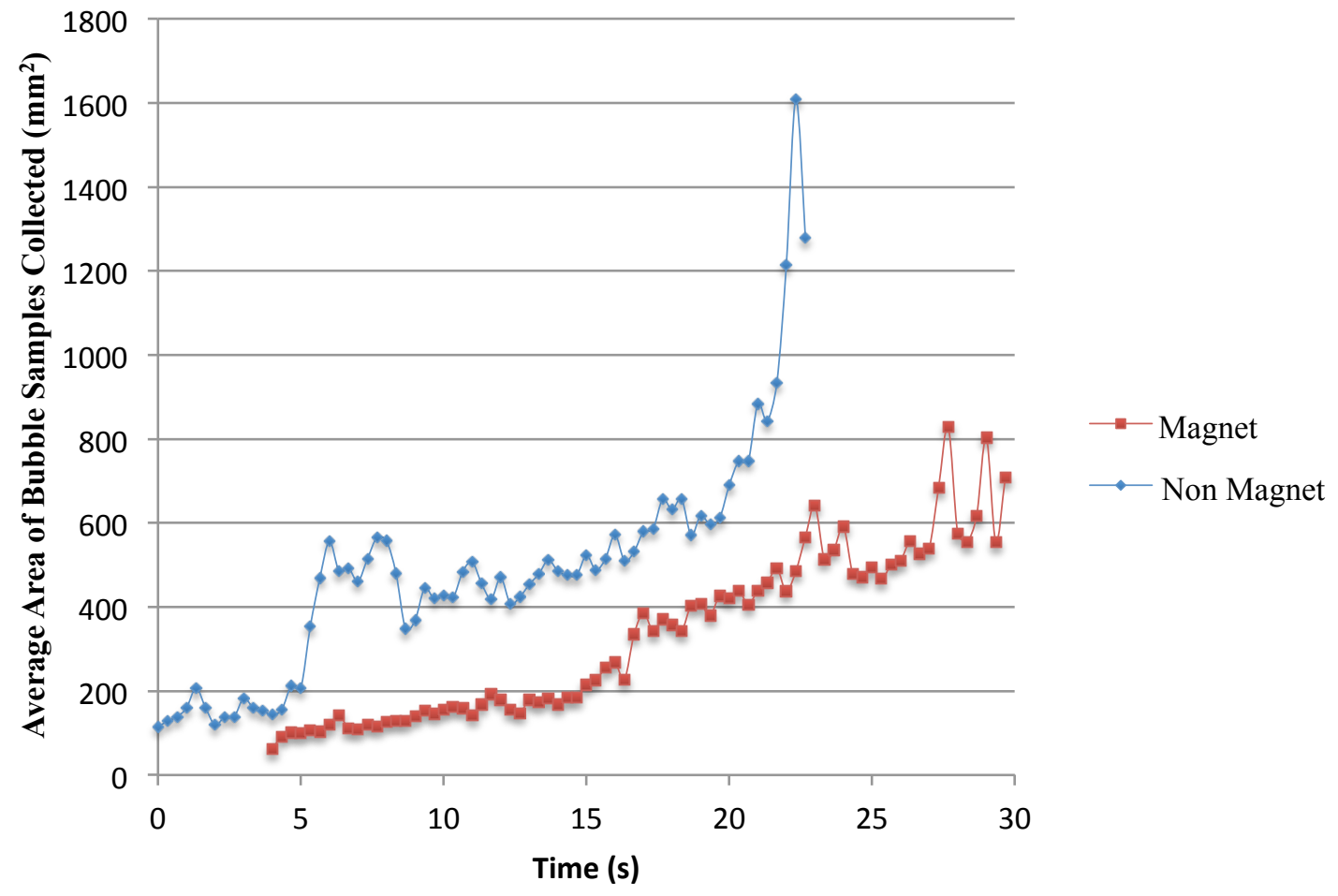

Figure 27: Comparison of Average Areas of Bubble Samples Collected from the Tank with and without the Magnet; Parabola 35

\section{Relationship Between Bubble Radius and Bubble Height}

The bubble radii values with respect to the Y-coordinate value were measured as the bubble moved from the bottom of the tank to the top of the tank. Average radius was then used to calculate the area of a bubble at the same height on magnet tank and non-magnet tank. The bubbles on the non-magnet tank tend to have higher radius compared to the bubble radii from the magnet tank. This size difference of the bubbles was observed from many frame samples from various parabolas. As can be seen is shown in Figure 28, in the tank with the magnet there is an upward trend in the bubble radius as it moves upwards in the tank. The expansion of the bubble can be evaluated as the bubble radii values increases as it traverses along its path to top of the tank. As the bubble travels through the three regions of interest along the vertical axes of the tank, it is possible to corroborate with the size of the bubble as demonstrated in Figure 25. Similarly Figure 29 demonstrates the equivalent data for the tank without the magnet; note that the non-magnet tank bubble radii tend to be larger than for the magnet tank. 


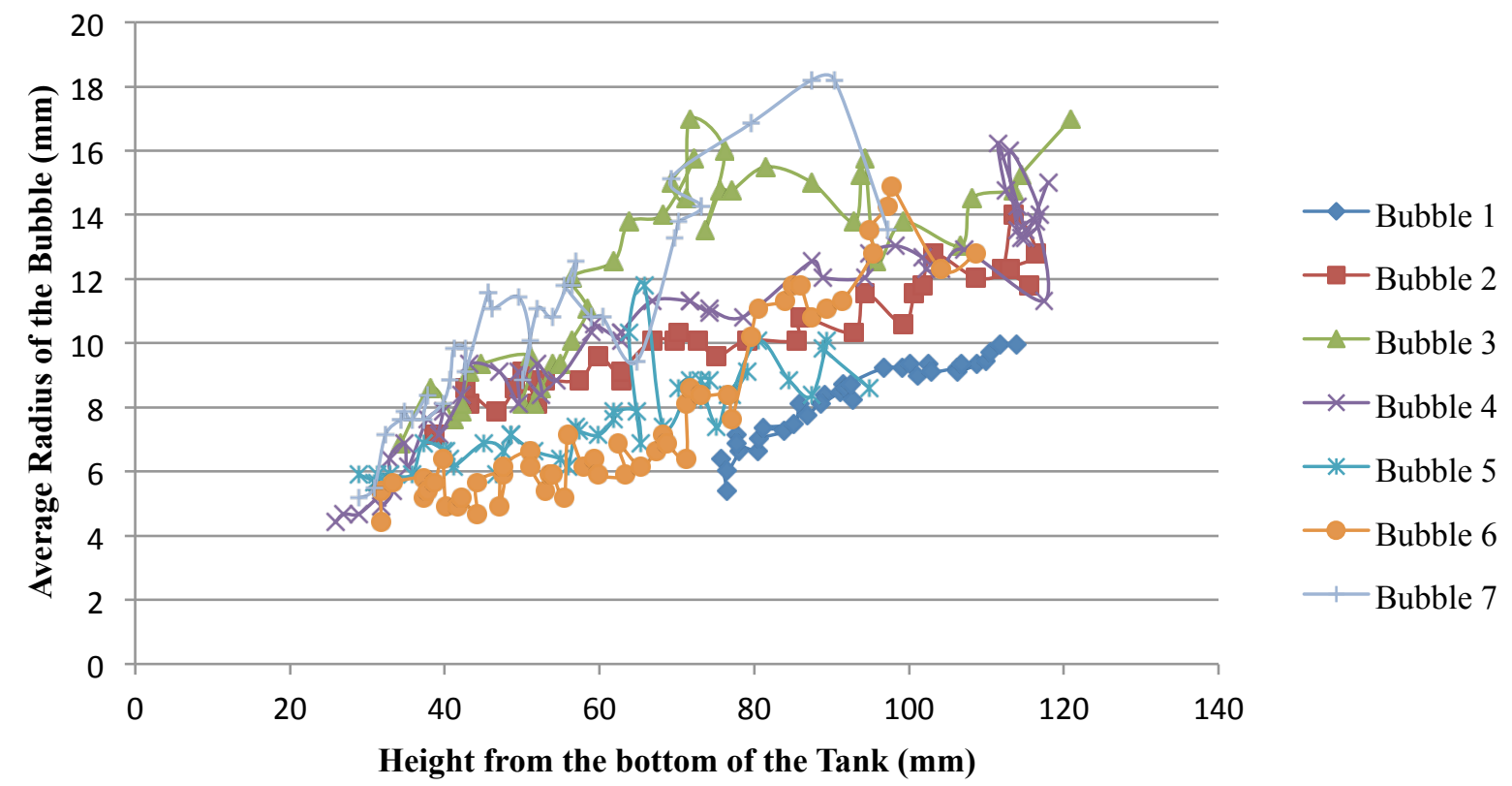

Figure 28: Average Bubble Radii vs. the Height from the bottom of the Tank (Magnet Tank); Parabola 35

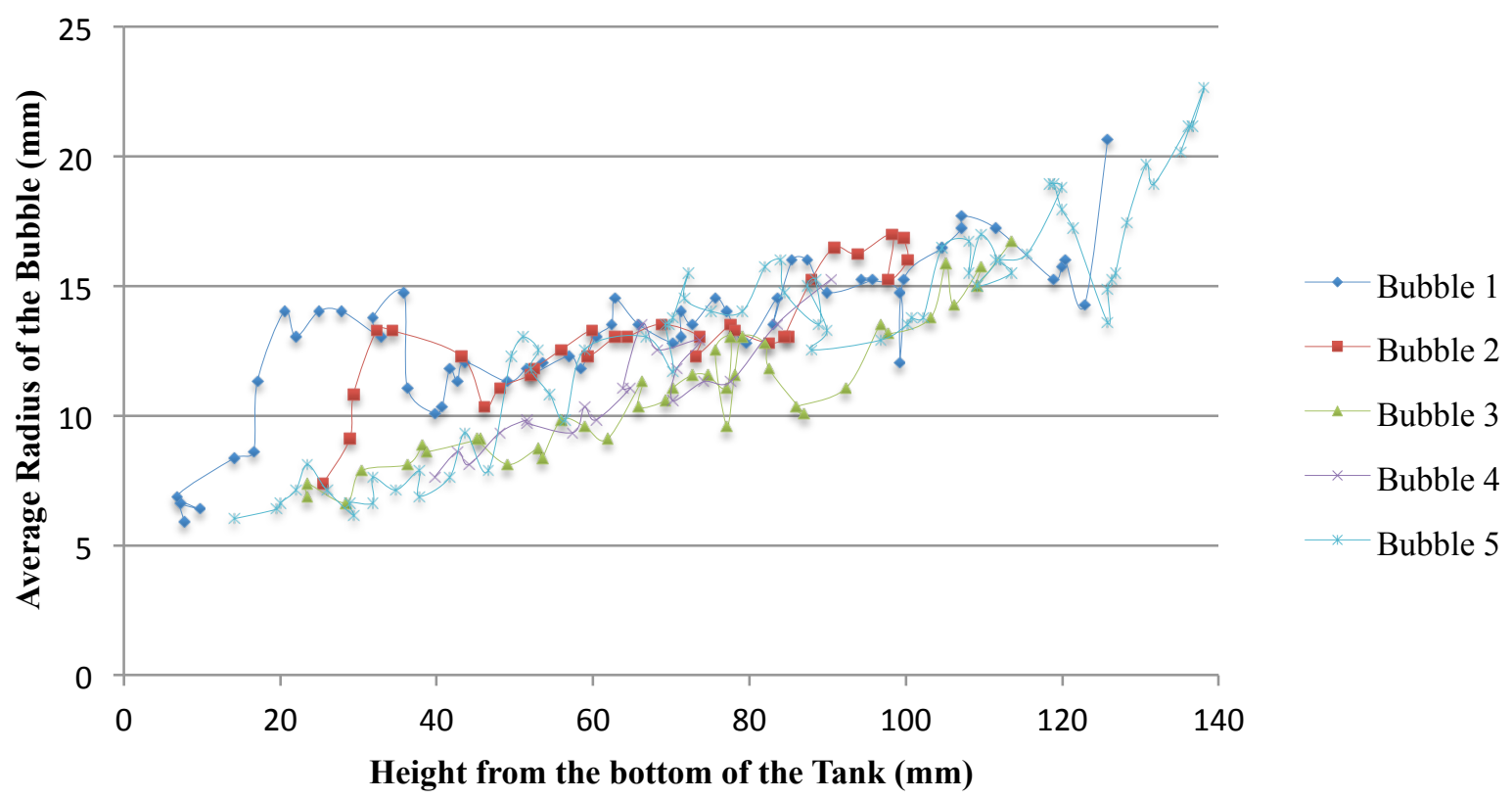

Figure 29: Average Bubble Radii vs. the Height from the bottom of the Tank (Non Magnet Tank); Parabola 35 


\section{Bubble Velocity}

In order to calculate the average velocity $\mathrm{v}_{\text {average }}$ of a chosen bubble, center coordinates of the same bubble were obtained from the consecutive image frames. The exact chosen bubble's coordinate across the image sequence with the constant frame rate, helps us to determine how fast the bubble is moving through the boiling liquid. Using the center coordinates, we can calculate the velocity in the horizontal direction known as the horizontal velocity component $u(\mathrm{~mm} / \mathrm{sec})$ and the velocity in the vertical direction, known as the vertical velocity component $v(\mathrm{~mm} / \mathrm{sec})$. The value difference of the $\mathrm{x}$ coordinates in two consecutive image frames is divided by the time between frames gives the $\mathrm{x}$ velocity component or the horizontal velocity component. Similarly, y velocity component or the vertical velocity component can be calculated. The following formulae were used in calculating the horizontal and vertical bubble velocities at a given frame $n$. The time between the every other frame, $\Delta \mathrm{t}$, was taken as $2 / 3$ seconds.

Equation 3: Horizontal Bubble Velocity Calculated Using the Central Approximations Method

$$
u_{n}=\frac{x_{n+1}-x_{n-1}}{2 \Delta t}
$$

Equation 4: Vertical Bubble Velocity Calculated Using the Central Approximations Method

$$
v_{n}=\frac{y_{n+1}-y_{n-1}}{2 \Delta t}
$$

Figure 30 shows the horizontal velocity of bubbles in the tank with the magnet for parabola 35 . There are lots of small fluctuations as all the bubbles seem to take a positive velocity followed by a negative velocity, indicating that the bubbles are not leaving their original x coordinate by a large margin. In contrast, the vertical velocity as shown in Figure 31 of almost all the bubbles takes a positive velocity. Bubble 4 seems to be an exception, and that could be due to the sudden movement of the tank that occurred when that bubble formed. The results from the non-magnet tank as can be seen in Figure 32 and Figure 33 respectively for the horizontal and vertical velocities, follow a similar pattern. Figure 34 shows the comparison of the horizontal velocities in the magnet and non-magnet tanks averaged over all seven bubbles in Figure 30 and the four bubbles in Figure 32, whereas Figure 35 shows the vertical velocities of the bubbles in the two tanks computed in a similar fashion by taking the averages of the bubble data from 
Figure 31 and Figure 33 respectively. From these graphs it is evident that the vertical velocity of bubbles in the non-magnet tank is higher than in the magnet tank, whereas the horizontal velocity remains somewhat similar.

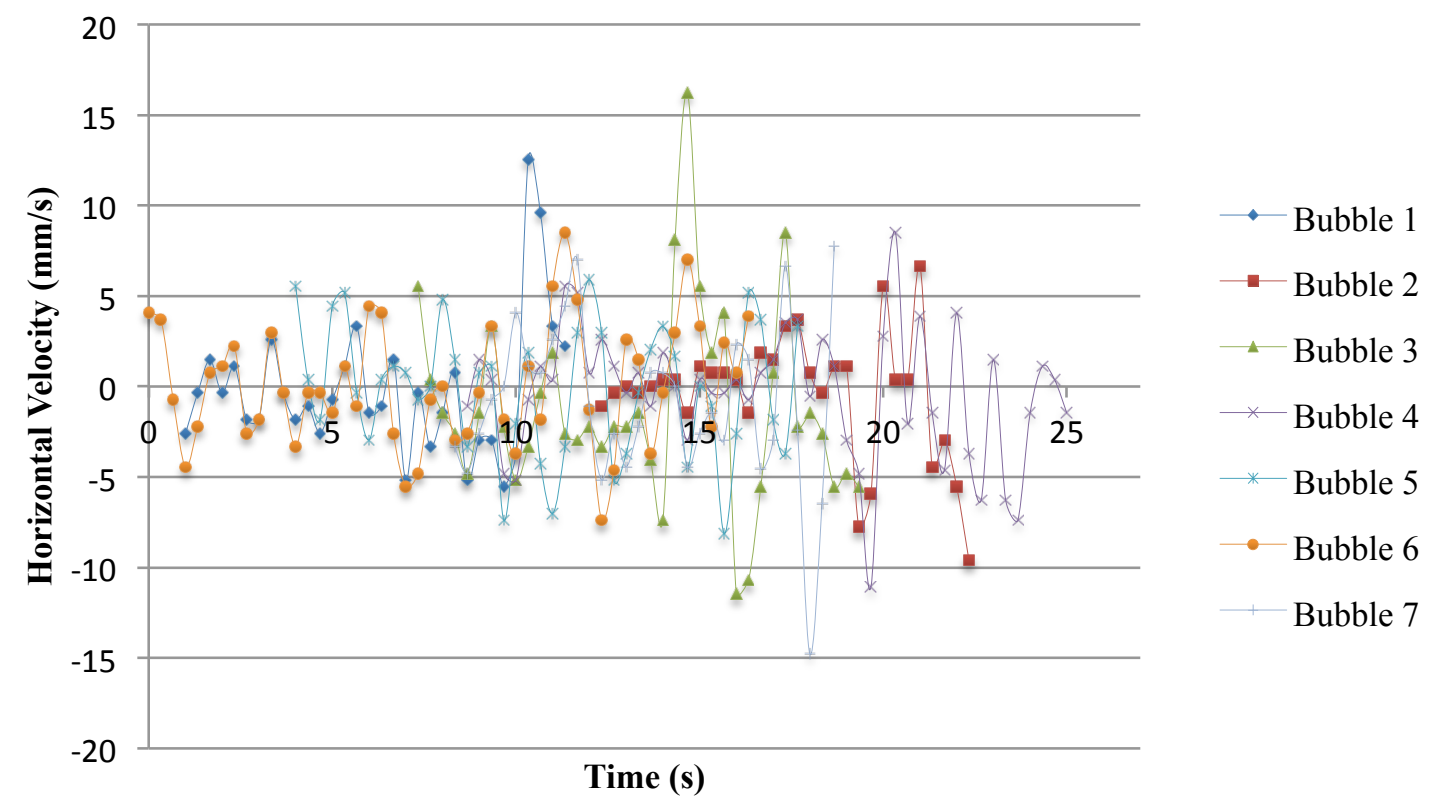

Figure 30: Visual Representation of the Horizontal Velocities of the Bubbles that are Located in Magnet Tank; Parabola 35 


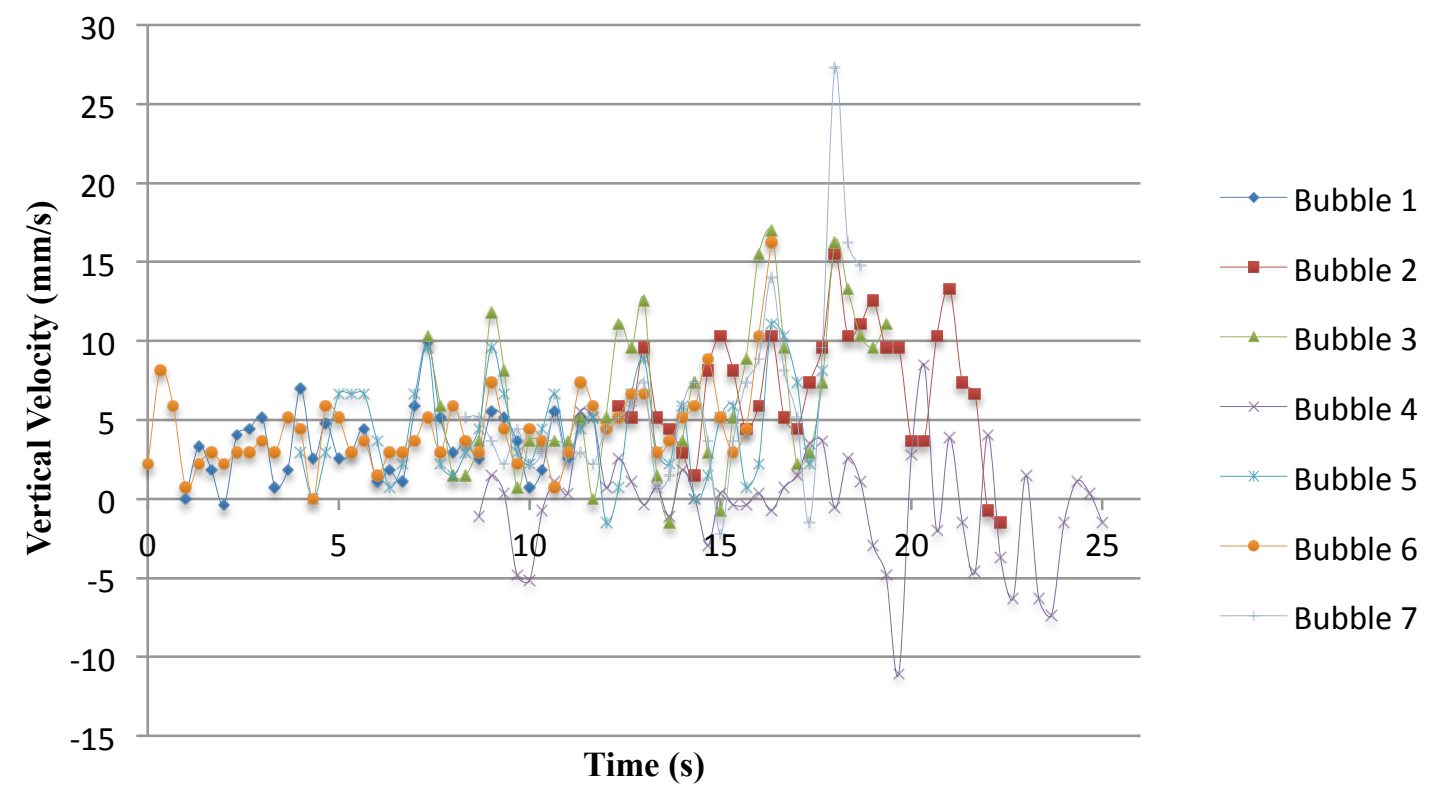

Figure 31: Vertical Velocity (Magnet Tank); Parabola 35.

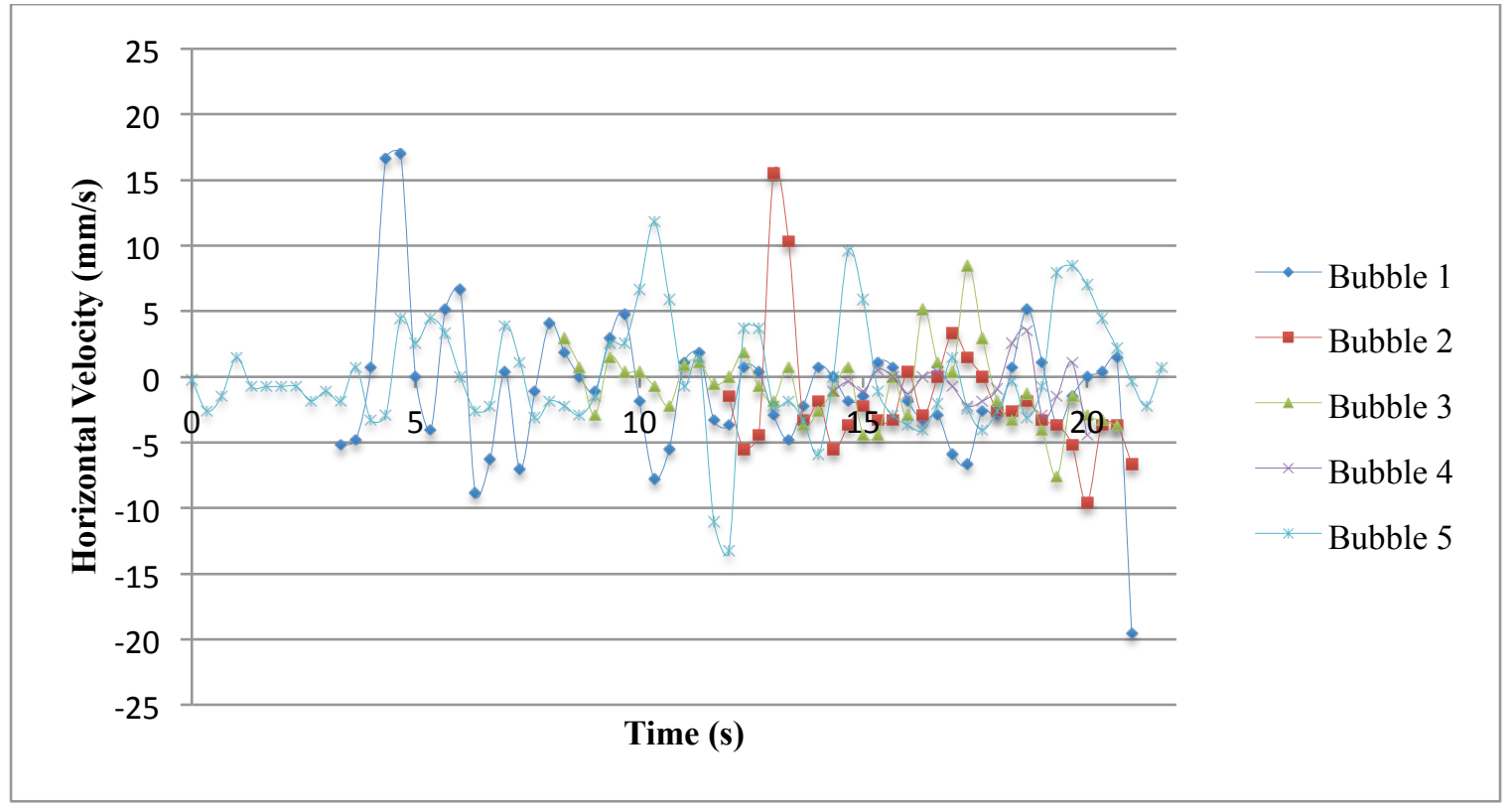

Figure 32: Horizontal Velocity (Non Magnet Tank); Parabola 35. 


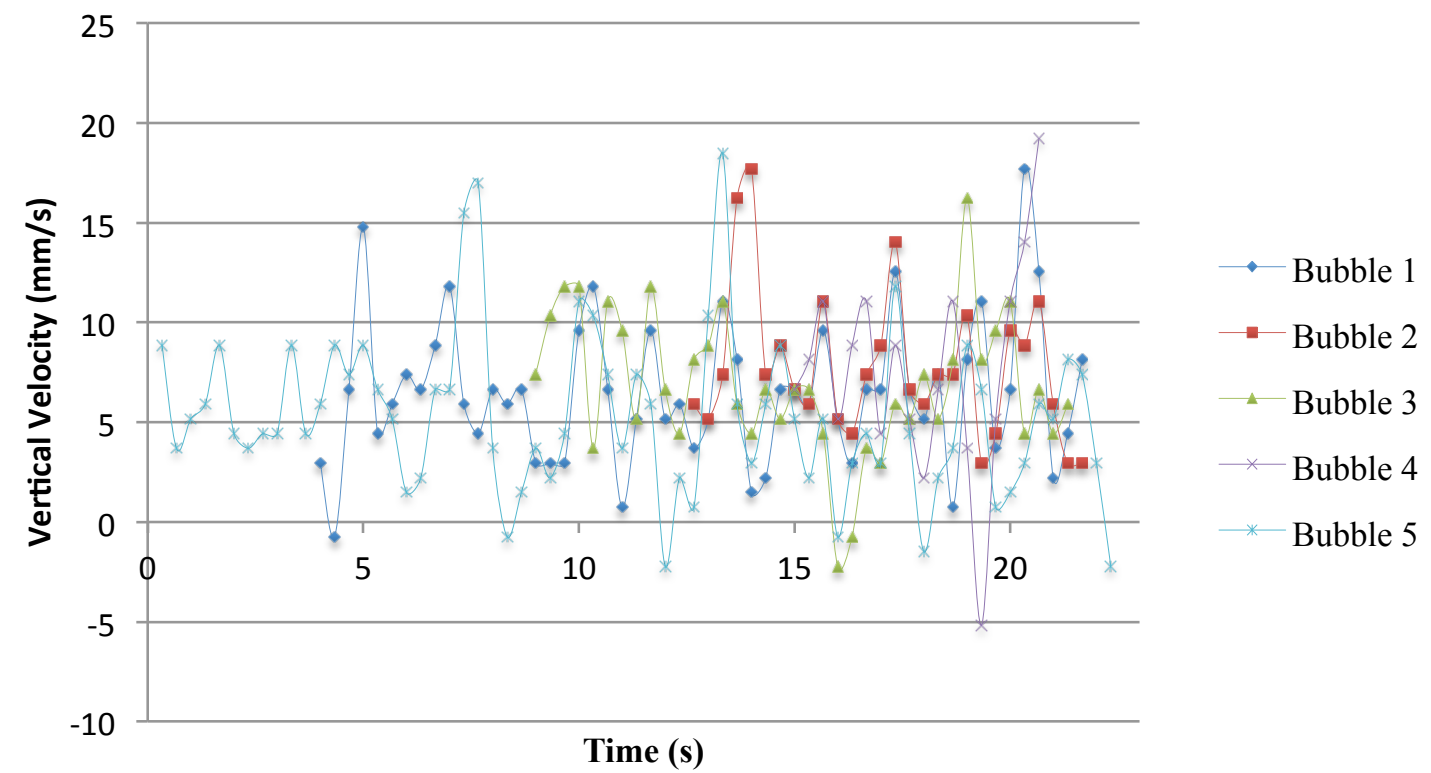

Figure 33: Vertical Velocity (Non Magnet Tank); Parabola 35

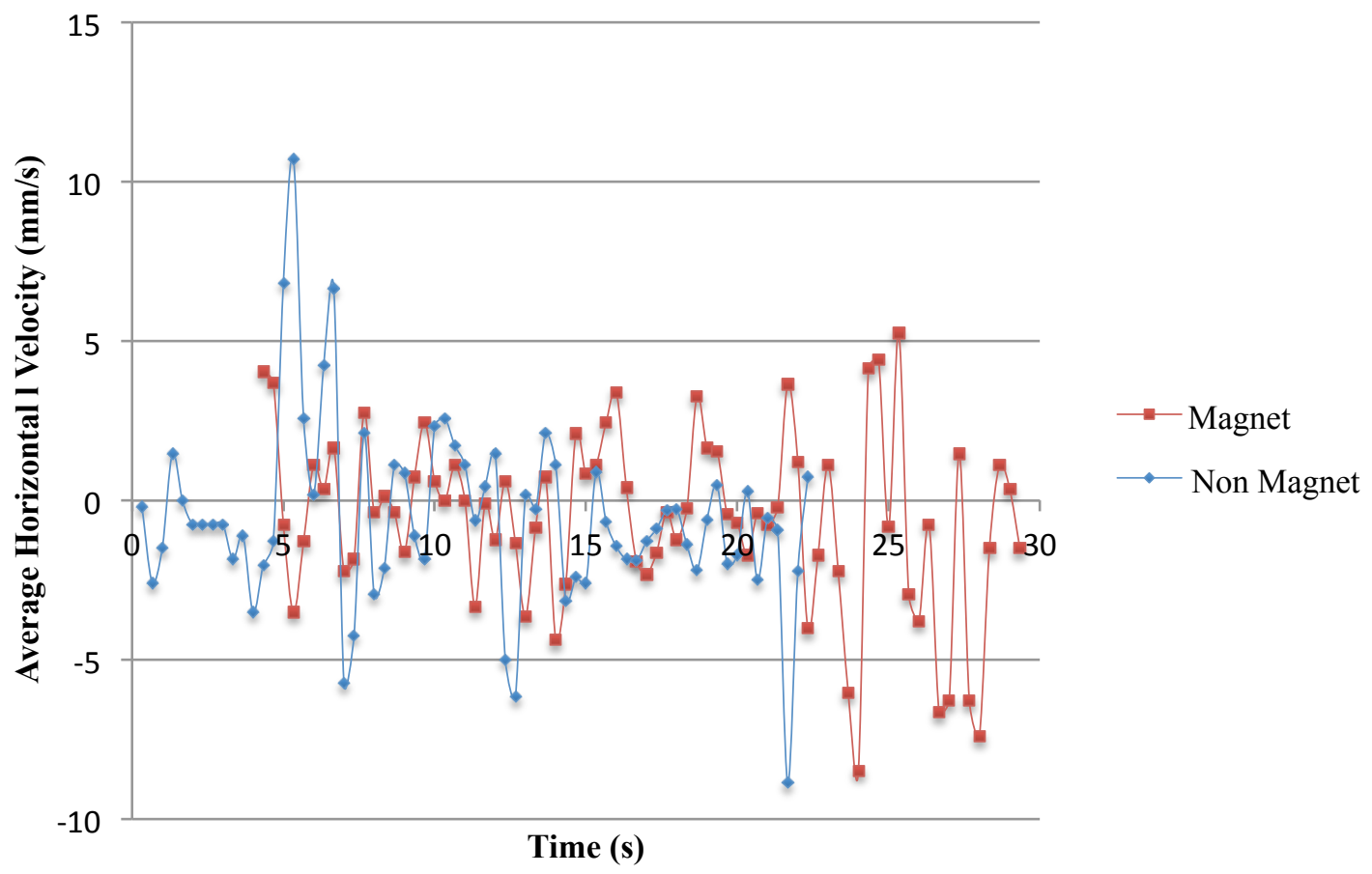

Figure 34: Comparison of the Average Horizontal Velocities in the Two Tanks; Parabola 35. 


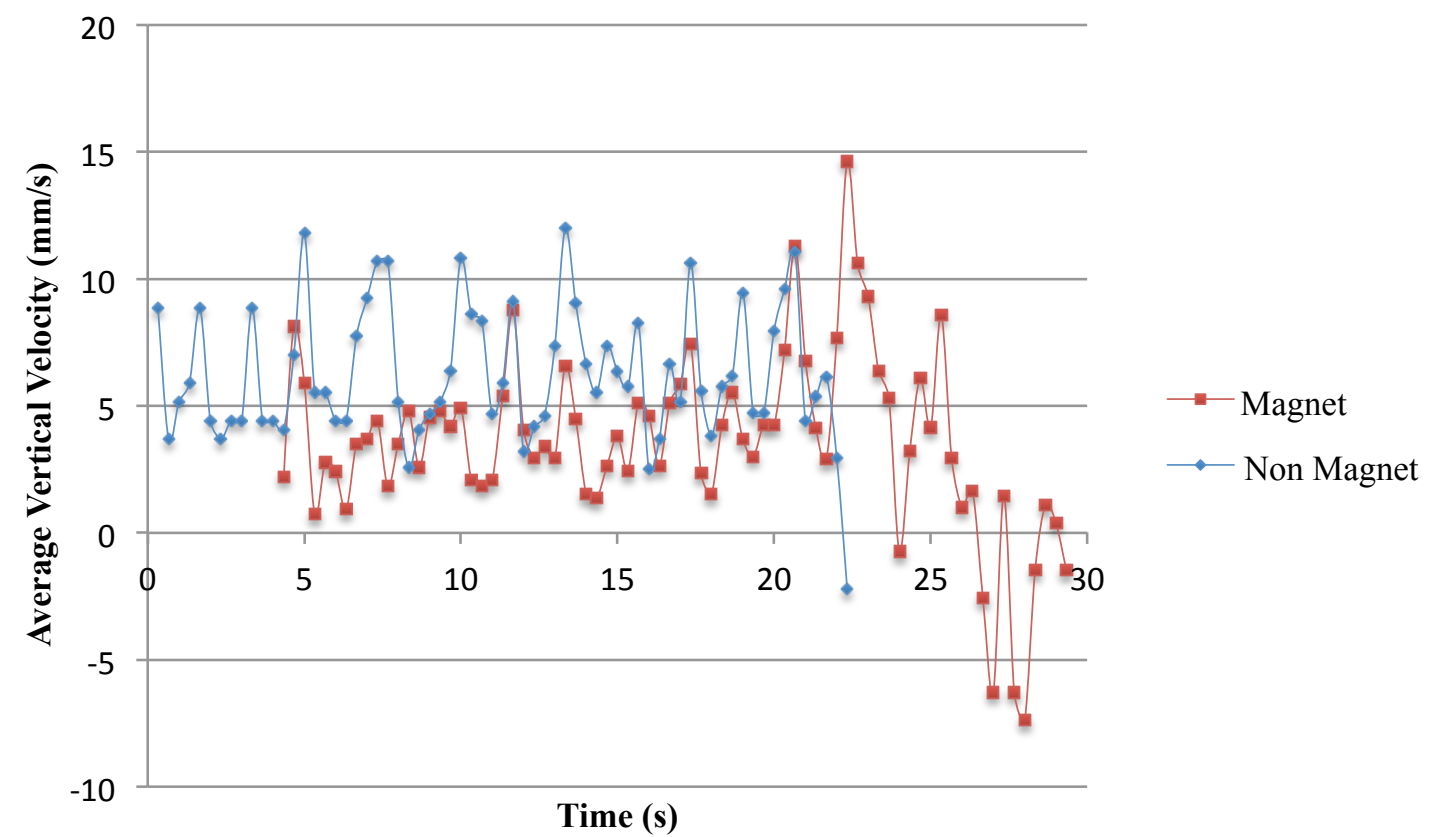

Figure 35: Comparison of Average Vertical Velocity in the Two Tanks; Parabola 35

Table 5 shows the mean, mode, median, variance and the standard deviation values obtained for the computed bubble velocities. Note that while the average horizontal velocities are both small and have similar magnitudes, both average vertical velocities are larger. The average vertical velocity for the nonmagnet tank is about $70 \%$ larger than the average vertical velocity for the magnet tank.

Table 5: Measure of Central Tendencies of the Bubble Velocities

\begin{tabular}{|c|c|c|c|c|c|}
\hline & Mean & Mode & Median & Variance & $\begin{array}{c}\text { Standard } \\
\text { Deviation }\end{array}$ \\
\hline $\begin{array}{c}\text { Horizontal } \\
\text { Velocity } \\
\text { (Magnet) }\end{array}$ & $-0.55 \mathrm{~mm} / \mathrm{s}$ & $1.11 \mathrm{~mm} / \mathrm{s}$ & $-0.37 \mathrm{~mm} / \mathrm{s}$ & $7.83 \mathrm{~mm} / \mathrm{s}$ & $2.80 \mathrm{~mm} / \mathrm{s}$ \\
\hline $\begin{array}{c}\text { Vertical } \\
\text { Velocity } \\
\text { (Magnet) }\end{array}$ & $3.59 \mathrm{~mm} / \mathrm{s}$ & $3.51 \mathrm{~mm} / \mathrm{s}$ & $3.60 \mathrm{~mm} / \mathrm{s}$ & $12.73 \mathrm{~mm} / \mathrm{s}$ & $3.57 \mathrm{~mm} / \mathrm{s}$ \\
\hline $\begin{array}{c}\text { Horizontal } \\
\text { Velocity (Non } \\
\text { Magnet) }\end{array}$ & $-0.52 \mathrm{~mm} / \mathrm{s}$ & $-0.74 \mathrm{~mm} / \mathrm{s}$ & $-0.74 \mathrm{~mm} / \mathrm{s}$ & $8.48 \mathrm{~mm} / \mathrm{s}$ & $2.91 \mathrm{~mm} / \mathrm{s}$ \\
\hline $\begin{array}{c}\text { Vertical } \\
\text { Velocity (Non } \\
\text { Magnet) }\end{array}$ & $6.24 \mathrm{~mm} / \mathrm{s}$ & $4.43 \mathrm{~mm} / \mathrm{s}$ & $5.61 \mathrm{~mm} / \mathrm{s}$ & $7.00 \mathrm{~mm} / \mathrm{s}$ & $2.65 \mathrm{~mm} / \mathrm{s}$ \\
\hline
\end{tabular}




\section{Bubble Trajectory}

With respect to bubble path during pool boing, Sanada et al. (2003) explain the behavior of a single column of small but equal size bubbles rising in a water column under Earth gravity conditions. They observe that the paths of bubbles had a zig-zag or helical component about the vertical axis that generally increased in magnitude as bubble diameter or bubble generation frequency was increased. The liquid jet generated by the bubble train also deviated further from the vertical direction as bubble size and frequency increased. We observed a somewhat similar behavior for the magnet tank where the horizontal displacement, as measured from the right wall of the tank, of the bubbles assumed a little bit of a zig-zag tendency as can be seen in Figure 36. In contrast, as can be seen in Figure 36, the bubbles formed in the non-magnet tank seem to oscillate to a greater extent, possibly due to the increased bubble size.

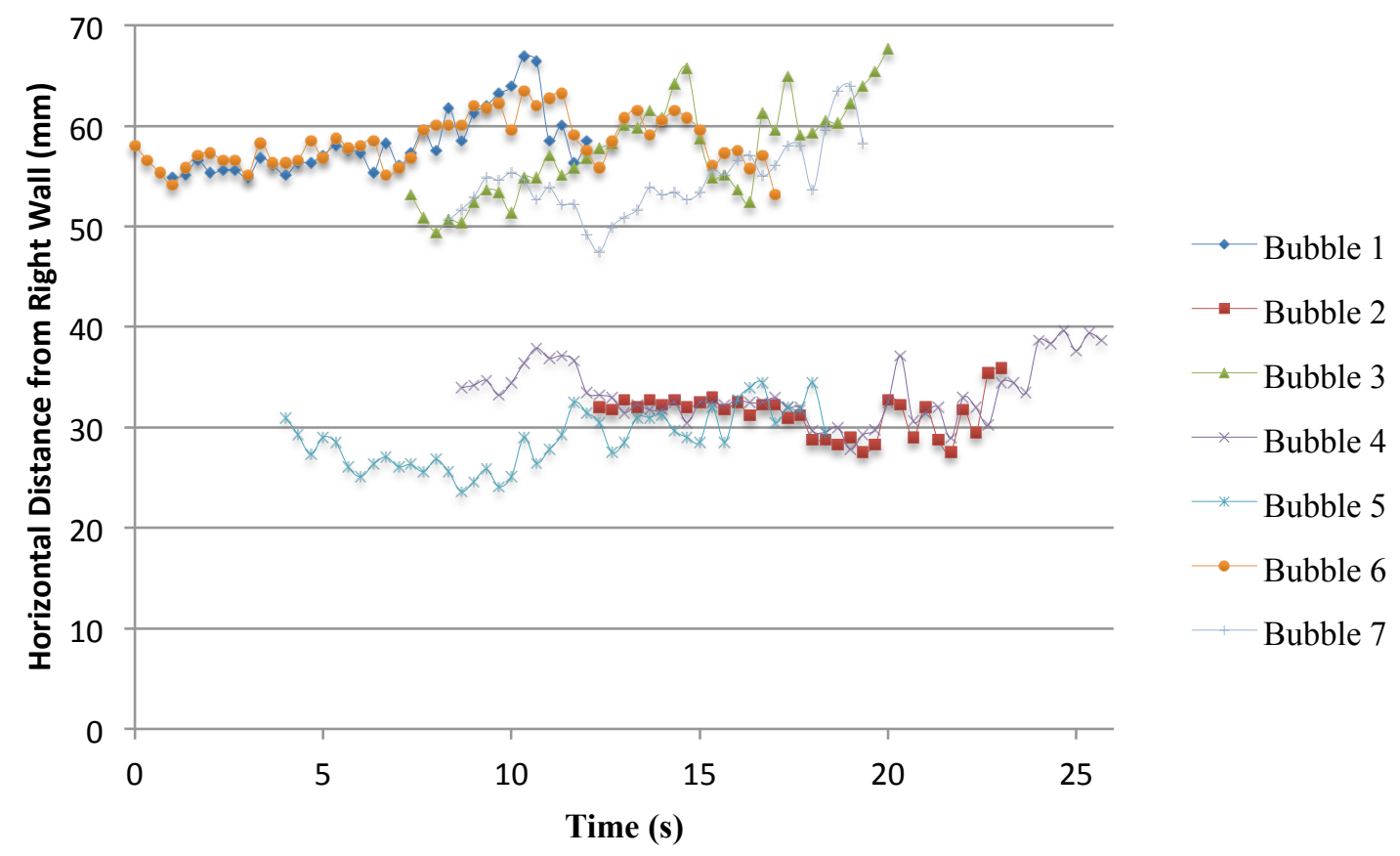

Figure 36: Horizontal Distance of Bubbles from Right Wall (Magnet Tank); Parabola 35 


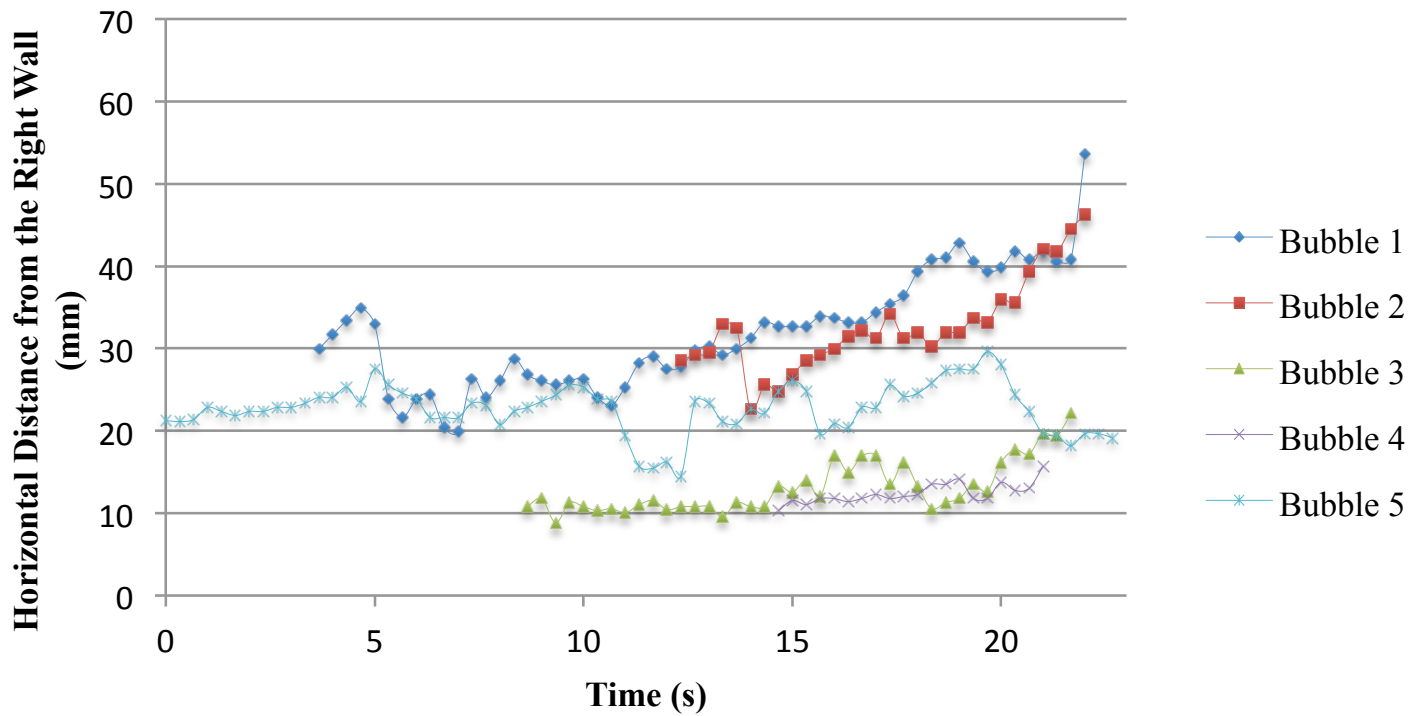

Figure 37: Horizontal Displacement of Bubbles (Non Magnet Tank); Parabola 35

The vertical distance of the bubbles from the heater surface increased as the time progressed. Figure 38 shows the vertical displacement of the bubbles in the magnet tank, whereas Figure 39 shows the vertical displacement of the bubbles in the non-magnet tank. In the non-magnet tank the vertical displacement was observed to be greater, which can be in part attributed to the larger bubble sizes. 


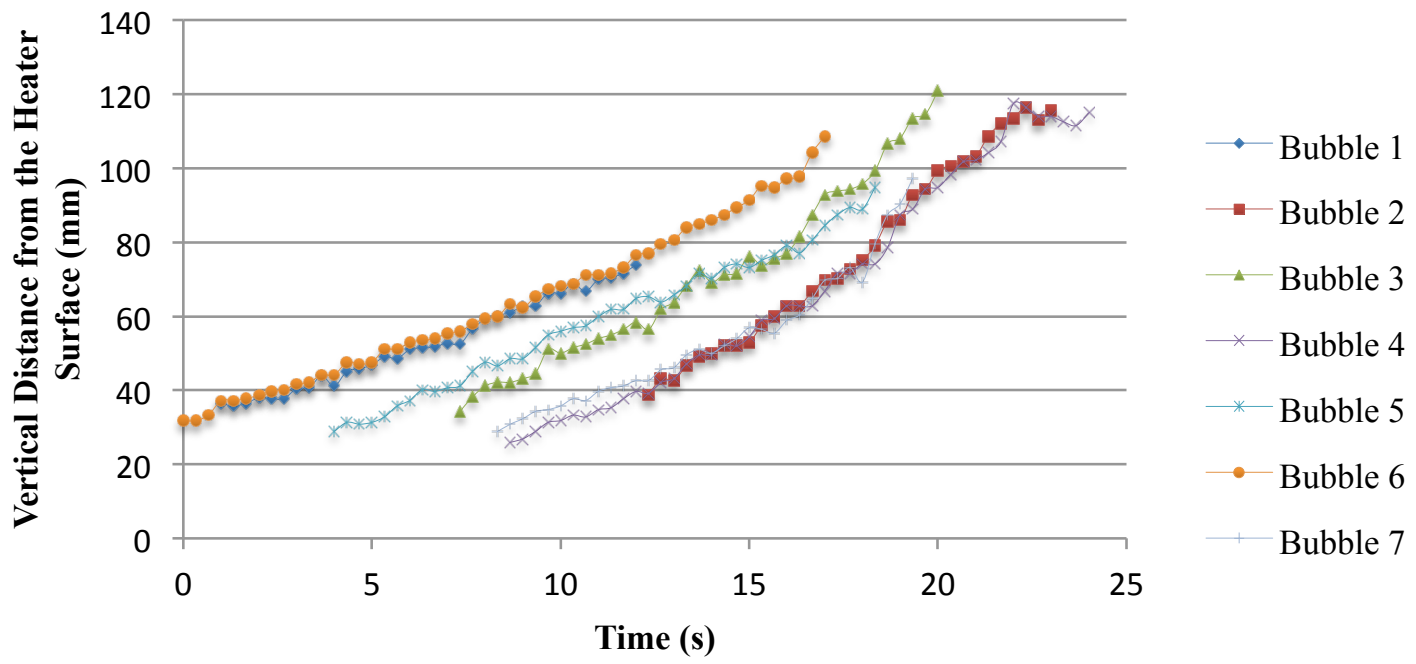

Figure 38: Vertical Distance of Bubbles from the Heater Surface (Magnet Tank); Parabola 35

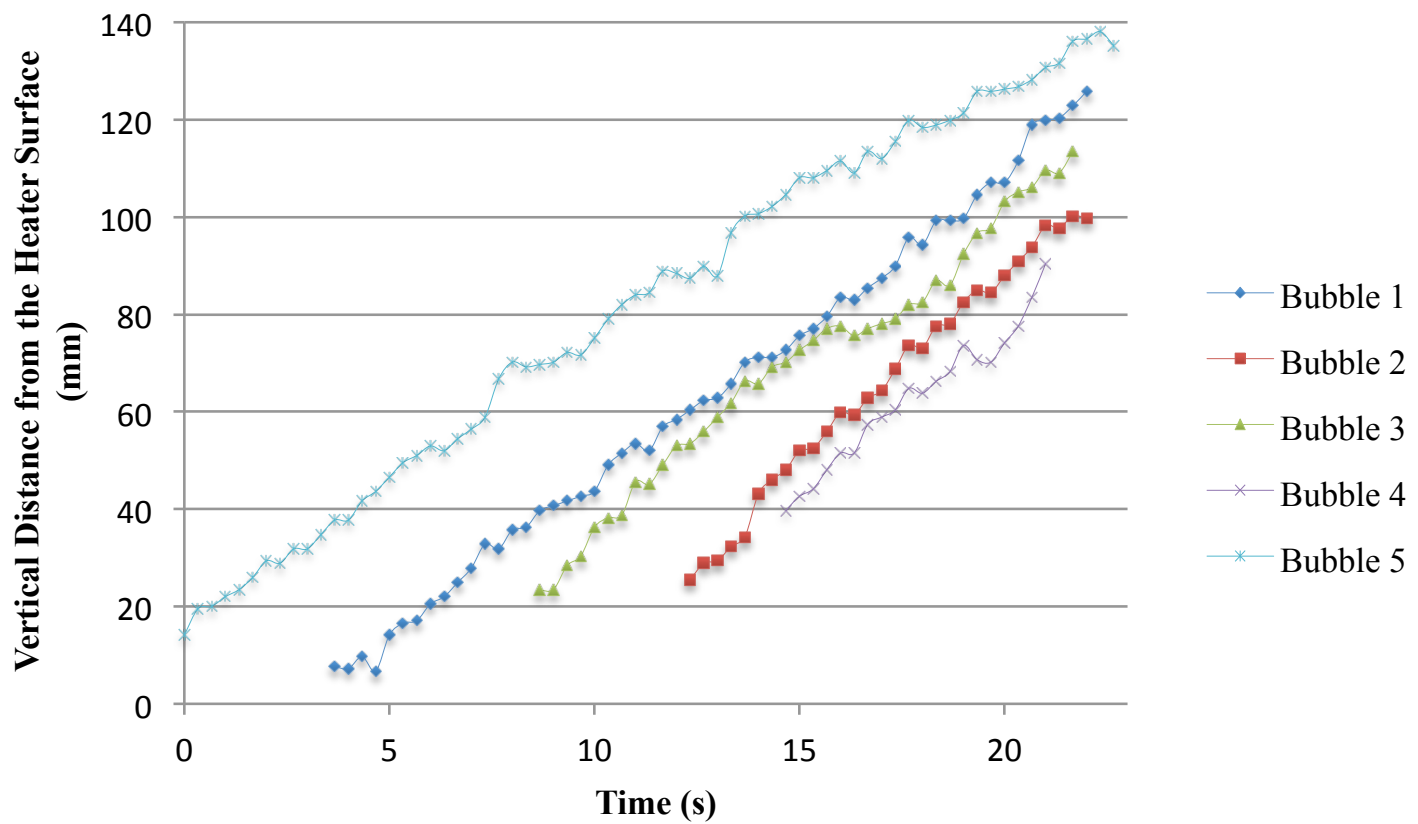

Figure 39: Vertical Distance of Bubbles from the Heater Surface (Non Magnet Tank); Parabola 35 


\section{Reynolds Number}

The bubble diameter and the velocity results were used to compute the dimensionless Reynolds number, which expresses the ratio of inertial forces to viscous forces. Inertial forces are characterized by the product of the density $\rho$ times the velocity $V$ times the gradient of the velocity $\mathrm{dV} / \mathrm{dx}$. The viscous forces are characterized by the dynamic viscosity coefficient $\mu$ times the second gradient of the velocity $\mathrm{d}^{2} \mathrm{~V} / \mathrm{dx}^{2}$. Defined from these first principles, the Reynolds number $(\mathrm{Re})$ is:

\section{Equation 5: Reynolds Number Definition}

$$
R e=\frac{\rho V \frac{d v}{d x}}{\mu \frac{d^{2} v}{d^{2} x}}
$$

The gradient of the velocity is proportional to the velocity divided by a length scale L. Similarly; the second derivative of the velocity is proportional to the velocity divided by the square of the length scale. Then:

\section{Equation 6: Reynolds Number Substitutions}

$$
R e=\frac{\rho V \frac{V}{L}}{\mu \frac{V}{L^{2}}}
$$

Further simplifying the above equation results in:

\section{Equation 7: Simplified Reynolds Number}

$$
R e=\frac{\rho V L}{\mu}
$$

The average velocity $V$, obtained from the bubbles from the tank with the magnet was 0.005106 $\mathrm{m} / \mathrm{s}(51.06 \mathrm{~mm} / \mathrm{s})$, and the average diameter of the bubble samples collected was $0.01960 \mathrm{~m}(19.60 \mathrm{~mm})$. According to the values used by Kuhlman and Gray (2007), at room temperature and at molarity of 4.5, the solution had a specific gravity of 1.4 , thus the density of the liquid being $1400 \mathrm{kgm}^{-3}$ and a viscosity of 0.005 Pa.s (5 cP). This results in a Reynolds Number of 28.02. In an experiment conducted by Kuhlman et al. (2005), but without the effect of heat as explained in this thesis, the Reynolds number was also found to be 28 . 


\section{Body Forces Acting on the Bubbles}

According to Kuhlman and Gray (2007), the Kelvin body force in terms of an effective gravity level is:

\section{Equation 8: Effective Gravity}

$$
g_{\text {effective }}=\frac{\chi_{s} B \nabla B}{\mu_{0} \rho_{s}}
$$

According to Andres (1976) and Mahajan et al. (1998), the solution magnetic susceptibility, $\chi_{s}$, was 0.000763 . The magnetic flux density, $B$, changes with the height of the tank as shown in Figure 7 , and $B \nabla B$ was taken as the resultant magnetic strength at any given height. $\mu_{0}$ is the permeability of free space, and is equal to $1.275 \times 10^{-6} \mathrm{~N} / \mathrm{A}^{2}$. The density; $\rho_{s}$, of the liquid was taken to be $1400 \mathrm{kgm}^{-3}$. Using Equation 8 with the above-mentioned values, the effective gravitational acceleration on the bubbles was calculated along the tank height away from the magnet. As can be seen in Figure 40, closer to the magnet the effective gravitational pull is the highest, and gradually decreasing along the height of the tank.

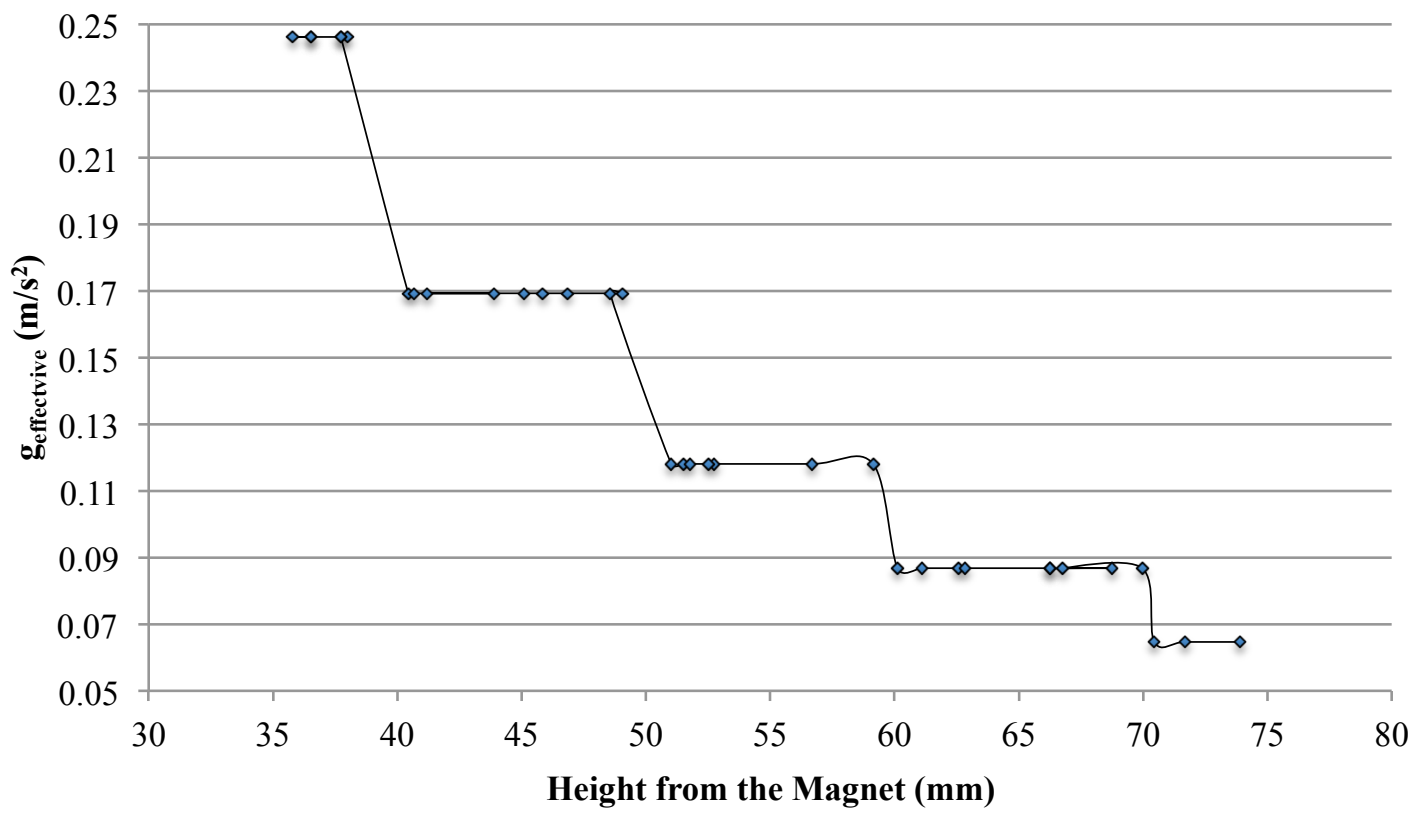

Figure 40: Effective Gravity; Parabola 35 


\section{Chapter 4: Conclusion}

Boiling is a very complex and illusive process due to the interrelation of numerous factors and effects such as the nucleation process, the growth of the bubbles, and the interaction between the heat source with the liquid and the vapor, the bubble departure process, the evaporation process at the liquidvapor interface, and the transport process of vapor and hot liquid away from the heat source. Although boiling mechanisms have been investigated under normal gravity conditions for some time, an understanding of thermo and fluid dynamics in boiling under reduced gravity is essential for the designers of future space applications that may involve critical aspects such as heat removal with boiling. Although much research in this area has been performed over several decades, many of the experiments show contradictory results, with some experiments showing no effect of gravity on heat transfer while other showing a strong correlation. These discrepancies can be due to the fact that some of the experiments are carried out within a very short duration, and effects such as convection may not be eliminated completely during this time.

\section{Research Outcomes}

Pool boiling of a paramagnetic liquid in two identical tanks was created under microgravity conditions to study the characteristic behavior of the vapor bubbles under influence of a magnetic field. The reduced gravity conditions in the experiment simulated the behavior in outer space environments.

At a fixed value of temperature and pressure with different gravity levels such as Earth gravity and microgravity, bubble behavior in pool boiling has different characteristics. Under the microgravity conditions, the boiling process inside the two tanks takes place suddenly in an explosive manner within a short period of time. This sudden increase in diameter and velocity of the bubbles at the bottom region of the tank was explained by analyzing the vertical and horizontal velocity components of the bubble. During this study, it was observed that the strength of the magnetic field had an influence on the bubble's size. Horizontal and vertical radius, bubble area, bubble velocities, and bubble path along the vertical axis of the tank was graphed verses bubble frame number (time) during this study. We were able to observe that the bubbles that were at the bottom of the tank had lower vertical radius than the horizontal radius. 
Also, the bubble size was comparatively smaller than the bubbles in the upper level of the magnet tank. These bubbles also were observed to be more elliptical in shape, with their vertical radius being smaller than the horizontal radius.

Thus results of the study indicate:

- Under the magnetic field influence, bubbles which were generated by pool boiling under the microgravity conditions are more circular in their cross sectional shape, and are smaller than in the non-magnet tank.

- On the other hand, bubbles without the magnetic field influence tend to have larger horizontal radius than the vertical radius, and are larger than in the magnet tank.

- Under the microgravity conditions, the boiling process inside the two tanks take place suddenly in an explosive manner within a short period of time as explained by the velocity graphs.

Most of the preliminary results analyzed using the methods described in the Appendix have been briefly summarized previously in the following peer-reviewed research papers:

- "Dynamic and Kinematic Characteristics of Bubble Flow Motion in Paramagnetic Liquid under Microgravity Conditions". International Conference on Fluid Flow Dynamics (ICFD), Sendai, Japan, Thilanka Munasinghe, November 2009.

- "Transformation Mapping of Bubbles' 2-D Circular Shape to an Elliptical Shape Under Influence of a Magnetic Field in Pool Boiling in Microgravity Conditions". 5th MIT Conference on Computational Fluid and Solid Mechanics, Massachusetts Institute of Technology (MIT), Cambridge, MA, Thilanka Munasinghe. June 2009.

- "Investigating Bubble Expansion in Pool Boiling Under Influence of Magnetic Field in Microgravity Conditions". World Scientific and Engineering Academy and Society, (WSEAS), Moscow, Russia, Thilanka Munasinghe, Sanket Joshi. August 2009.

- "Studying the Characteristics of Bubble Motion in Pool Boiling in Microgravity Conditions Under the Influence of a Magnetic Field". Recent Advances on Space Technology (RAST), IEEE - AIAA Joint Conference, Istanbul, Turkey. Thilanka Munasinghe. June 2009.

- "Investigating Bubble Behavior in Pool Boiling in Microgravity Conditions," World Congress on Engineering (WCE), International Association of Engineers (IAENG), Imperial College, London, UK, Thilanka Munasinghe, July 2008. 


\section{Appendix: Preliminary Methods Used for Image Processing}

In this particular study, image-processing methods enabled us to quantify the characteristics of the bubble behavior in pool boiling in a paramagnetic liquid under microgravity conditions. The Matlab Image processing toolbox was initially used in developing these processing methods. In order to learn the Matlab Image processing tools and how to use them efficiently and adequately, several tutorials and some textbooks related to image processing using Matlab have been referenced. This includes the Matlab tutorials that are available under the help command, and the "Digital Image Processing using Matlab" by Gonzalez and Woods (2 ${ }^{\text {nd }}$ Edition, 2002). Some of the free online tutorials were also used in some particular areas such as developing image arrays, matrices and loops for both image and some parts of the video processing.

\section{Image Processing by using Matlab® "Image Processing Toolbox"}

Using the image processing toolbox in Matlab, characteristics of the bubbles that are appearing in the images have been studied. At the beginning of the present work, the special commands that are needed in the image processing toolbox needed to be learned. Self-study of Matlab image processing functions and commands and their special features that are available in the toolbox were undertaken using the Matlab help menu. In addition to that, online Matlab tutorials provided the required knowledge to conduct the image processing of the pool boiling images. Once the meanings of the Matlab functions and their desired features had been mastered, with many practice attempts, finally it was possible to conduct the image processing to determine the information and the characteristics of the bubbles that were appearing in the images.

In order to capture the images that are important to study the bubble behavior, image frames were captured from the video clips of pool boiling obtained from microgravity flight. The video was $30 \mathrm{fps}$, and each $6^{\text {th }}$ frame was selected for processing, giving the time between the selected frames to be 0.2 seconds. In order to capture the image frames with constant time gap between the images, we carefully selected frames with the highest visibility of the bubbles. The image frames that have been captured from the video clips were imported to the Matlab working directory and stored in the Matlab working folder to export the images to the Matlab "workspace" when required. After exporting the images to the 
"workspace" in Matlab, those images were converted to the "jpeg" or "tiff" images. After converting the images; those images were stored as M-files for future reference in order to study the characteristic details of the image information. Next step was to conduct the image filtering, where the images that are in color scales of red, blue, green (RGB) were converted to the black and white gray scale for the better visibility of identifying the bubbles. Later on, converting images from color to black and white and gray color images using Matlab commands such as "rgb2gray" and also converting color images to binary images using rgb2bi Matlab commands were done.

Some of the images needed to be cropped from the original image, and this was done using the "imcrop" command. It was also useful to change the color contrast of the black and white images in the gray scale and look for the better visibility to detect some of the bubbles that were not seen in the images before.

Histogram equalization was performed using the imtool command. Image frame was given in a Cartesian coordinate system to get the $\mathrm{x}$-coordinate and the $\mathrm{y}$-coordinate of a location on the image. The main reason to give a coordinate system is to identity the location and size of the bubble in that coordinate system. In Matlab toolbox, there is a command to pick up the location points on image by using "ginput" command. Using that command, the bubble locations were determined and saved into the computer memory. After determining the bubble locations in the consecutive bubble frames, the bubble path was determined, and it was useful to create the vector lines of the bubbles positions and vector maps. Bubble velocity components in the "X" and " $Y$ " directions were calculated and plotted on a graph. Also the bubble's vertical and horizontal radii were determined to calculate the bubble area.

Types of Images Used In Image Processing:

1. Binary images (Black and White),

2. True color images (RGB),

3. Gray scale or Intensity images, and

4. Indexed image.

\section{Useful commands:}

One of the images gathered from the pool boiling video clip has been used here to demonstrate how the image processing was done to analyze the bubble behavior. Therefore, the image frame number 
28 has been arbitrarily chosen. This original image that captured from the video clip was saved as the 28.tiff in the Matlab working directory with the extension of "tiff" designating the image format. The number 28 represents the image frame number and that frame number was used to identify and save the image in the Matlab working directory. The numerical values relevant to the frame number were used for the convenience and the simplicity of the process. Using the "imread" command we performed the digitization process of the image. During this process, the image has been recognized and read into the Matlab environment with the name given by the user. Then it was stored as a matrix in the workspace with the assigned name. In the example below, the name "I" was given to the image during the reading process. In order to show the image "I", the Matlab command "imshow" was used. As a result of that, the image has shown in a new Figure 41 as shown below.

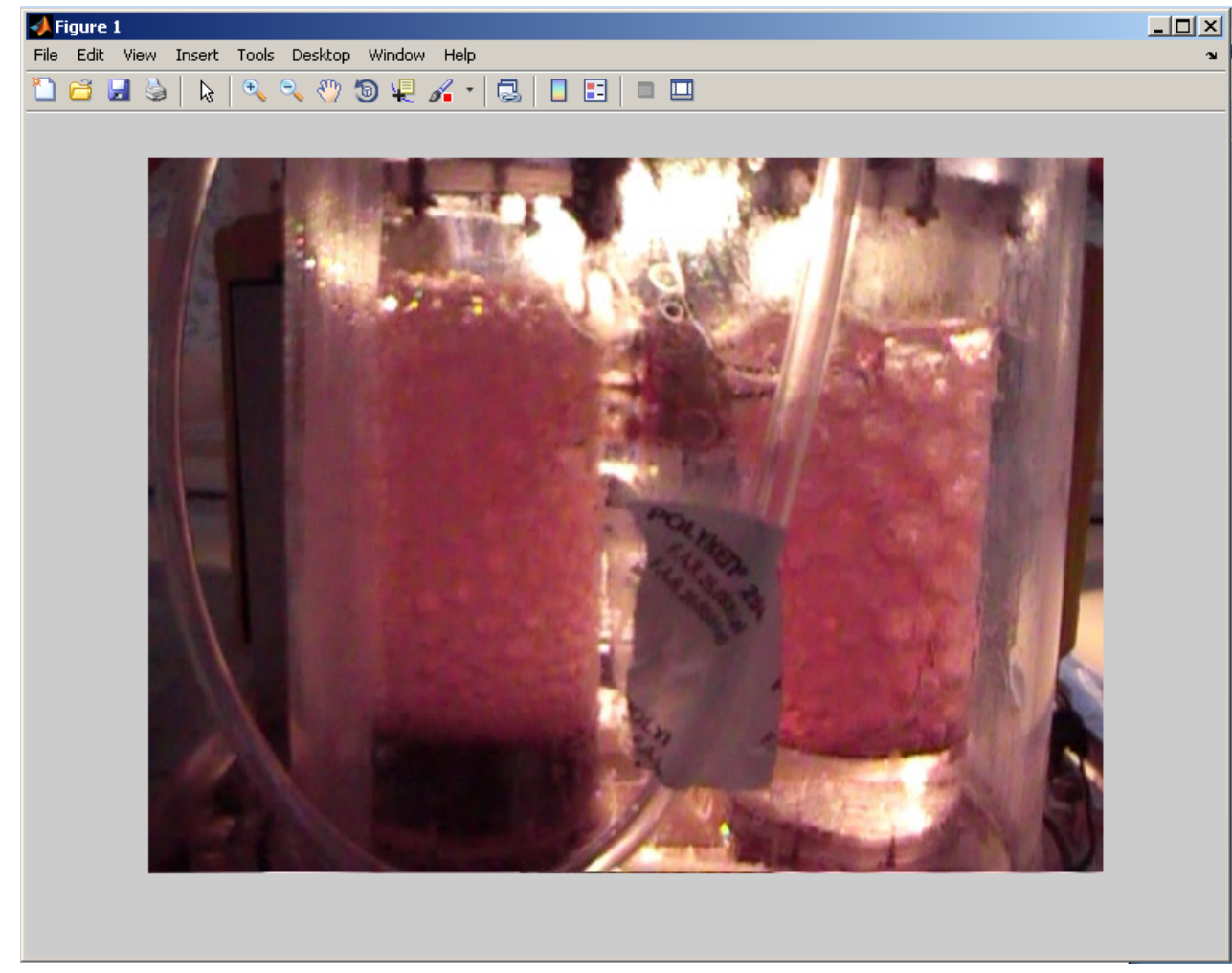

Figure 41: Image digitized and imported to Matlab

\section{Reading Images:}

$$
\begin{aligned}
& I=\text { imread('28.tif'); } \\
& \text { imshow(I) }
\end{aligned}
$$


The image 28.tiff is saved in the Matlab working directory and it has been read using the "imread" function and assigned the variable name "I" into that digitized image. The "imshow" function was used to show the digitized image as demonstrated below in Figure 42.

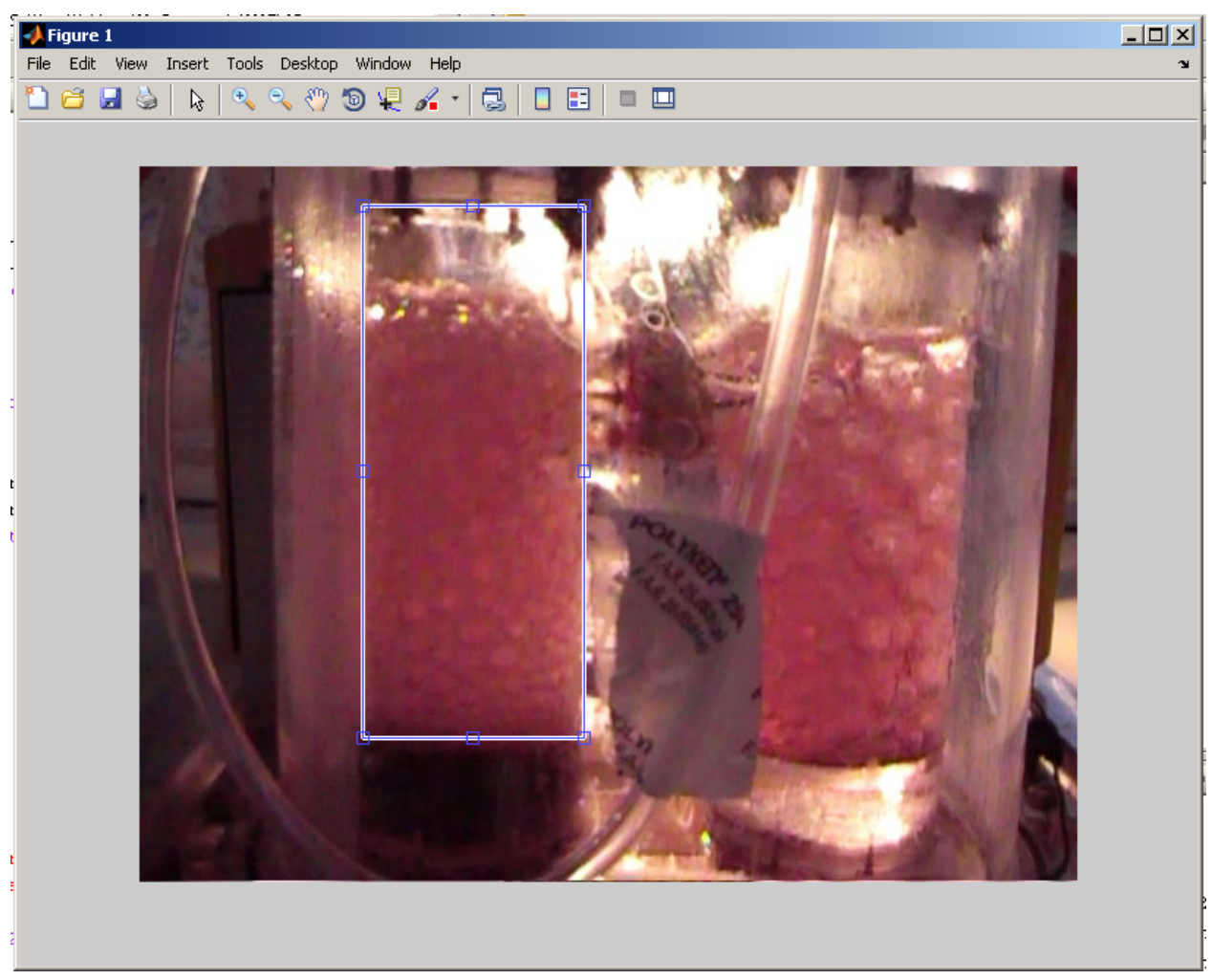

Figure 42: Cropping an Image

\section{Writing Images}

The function "imwrite" writes an image to a file that is assigned with a specific format. This function is useful to convert an image to a different format. File extension of the image can be identified along with the image name.

bubble=imread('frame28.png');

imwrite(bubble,'newimage.tiff'); 
The above expression will write the image frame 28 to a new file in "tiff" format with the name of "newimage" in the Matlab working directory. Therefore we can save this new image for our future references as needed.

\section{Cropping Images}

The next step was to crop the image. It was required to crop the image to focus on the tank area to study the bubbles. The Matlab "imcrop" command was used for this process. Images can be cropped manually or given by the coordinates of the rectangular region relevant to the coordinate values of the image. In this case, images were carefully cropped by the manual method by using the mouse cursor to locate the rectangular area of interest, as shown in Figure 42.

When these Matlab statements were executed in the command window, the image was cropped since it was the immediate image that was generated by Matlab. (Usually, by default, Matlab will choose to crop the image that appears in the figure that was immediately opened before the "imcrop" command was executed in the command window unless the user specifically identified it.) Once the image has been cropped into the size that was assigned by the rectangular area, a new figure will be displayed.

During the cropping process, the new image as identified with the name "c", and it has been automatically stored in the workspace of Matlab. The code used to crop the image has been shown below. Later, this cropped image is saved in the Matlab working directory with a given new name of "c1"under the extension of "tiff".

$$
\mathrm{c}=\operatorname{imcrop}(\mathrm{I}) \text {; }
$$

figure;

$\operatorname{imshow}(\mathrm{c})$

\section{Converting True Color Images to Gray Scale Images}

The true color images also are known as the RGB images since they are combined with the layers of the Red, Green and Blue color layers. The RGB abbreviation is given relevant to the initial letter of the 
each color layer. By using the "rgb2gray" command in Matlab, the true color images of the pool boiling have been converted to the gray scale images. This process was undertaken in order to recognize the locations of the bubbles more clearly. As a result of that borders of the bubbles were much more visible in the gray scaled images. Also, it was useful to recognize some hidden bubbles in between groups of bubbles that were not clearly visible in the true color scaled images.

$$
\begin{gathered}
\mathrm{G} 1=\operatorname{rgb} 2 \operatorname{gray}(\mathrm{I} 1) \\
\text { imshow }(\mathrm{G} 1)
\end{gathered}
$$

The converted gray scale image is shown in Figure 43.

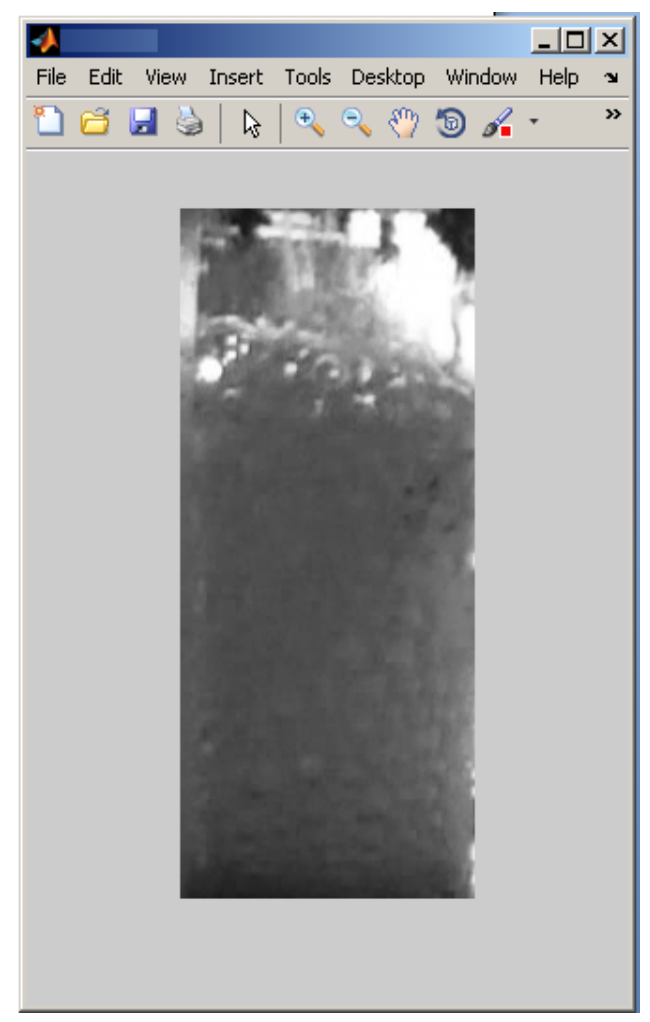

Figure 43: RGB to Gray Scale

\section{Using the "imtool" Command to Explore the Image Data.}

Once the images have been converted to the gray scale images, those images can be analyzed by using the "imtool" command. The "imtool" command is a very useful command to perform multiple tasks 
using the images. Once the image is opened from the imtool command, it shows the pixel information at the bottom of the figure, and it is also possible to determine the pixel regions by opening a separate figure. When the mouse cursor is moved on the main image panel, the gray level values of the pixels in the pixel region box will be shown relevant to the location of the mouse cursor as it moves on the main image. One of the other features of the imtool command is the "overview" figure. The overview figure shows the original image in a separate box with a rectangular box on the image and that rectangular box can be moved along the overview image on the figure, and as a result of the movement of the rectangular box, the image on the main figure also move along view panel. This is very useful for analyzing images that are large in size; see Figure 44 (imoverview), and Figure 45 (imtool). Under the imtool command it is possible to adjust the contrast and the brightness of the image by using the scroll bar. Also, image contrast or the brightness can be adjusted by using the scroll bar simultaneously analyzing the image "histogram" as will be demonstrated below.

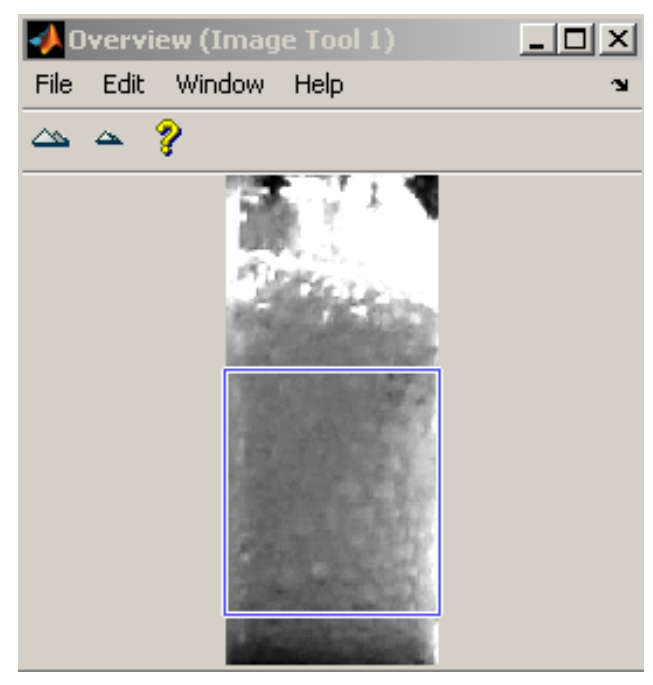

Figure 44: Output Display of the "imoverview" Command

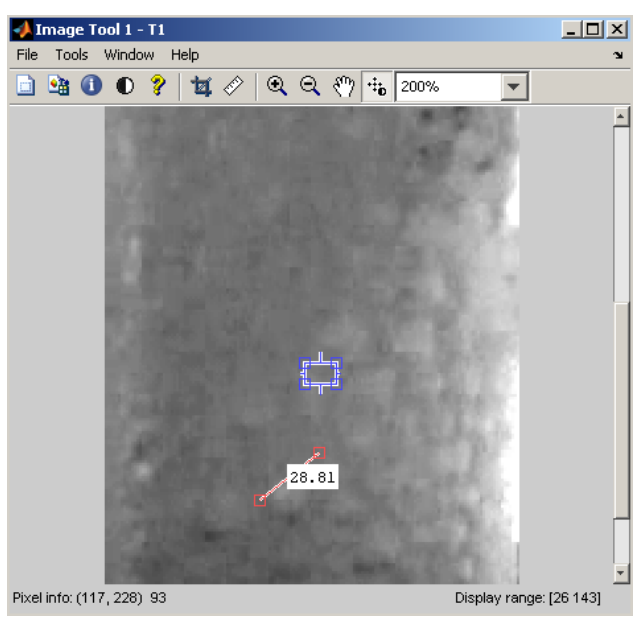

Figure 45: Output Display of the "imtool" Command

Under the "imtool" feature, it is also possible to determine the distance between two points, as demonstrated in Figure 45. The red square boxes represent the end point locations of the line segment and the distance between those two points is automatically calculated in pixel values and displayed in a small box. Clicking the mouse button on the image picked those two location points. This tool was very convenient to gather the vertical and horizontal radii of the bubble from the center point of the bubble. Since it was immediately displayed on the screen without any calculations conducted by the user, this special feature that comes under the "imtool" command helped save time, effort, and especially the 
accuracy of the data, as any manual calculations by using only the coordinate point values could introduce errors.

The blue box appearing in Figure 45 above can be moved along with the mouse cursor and the pixel information that is relevant to the position of the center of the box is shown on the left bottom corner of the figure as "Pixel info". Also, the display range of the image can be seen on the bottom right hand side of the Figure. The pixel value of the each coordinate point on a selected region in a gray scale image can be determined by using the "Pixel Region" image tool as shown in Figure 46.

\begin{tabular}{|c|c|c|c|c|c|c|c|c|c|c|c|c|c|}
\hline \multicolumn{10}{|c|}{ A Pikel Region (Image Tool 1) } & \multicolumn{2}{|c|}{$-\mid \underline{-} \underline{x}$} & AImage Information (Image Tool 1) & \multirow[t]{2}{*}{$-(\square) x$} \\
\hline File & Edit & Winc & low & Help & & & & & & & & & \\
\hline \multicolumn{12}{|c|}{${ }^{\dagger} \mathbb{t}^{-}$䧃? } & \multirow{2}{*}{\multicolumn{2}{|c|}{ Image details (Image Tool 1 - T1) }} \\
\hline 80 & 79 & 82 & 82 & 82 & 81 & 80 & 79 & 79 & 79 & 78 & 79 & & \\
\hline \multirow{2}{*}{81} & \multirow{2}{*}{81} & \multirow{2}{*}{82} & \multirow{2}{*}{82} & \multirow{2}{*}{82} & \multirow{2}{*}{81} & \multirow{2}{*}{80} & \multirow{2}{*}{79} & \multirow{2}{*}{79} & \multirow{2}{*}{79} & \multirow{2}{*}{78} & \multirow{2}{*}{79} & Attribute & 1 \\
\hline & & & & & & & & & & & & Width (columns) & 157 \\
\hline 83 & 82 & 81 & 81 & 81 & 80 & 80 & 79 & 79 & 79 & 76 & 79 & Height (rows) & 362 \\
\hline \multirow{2}{*}{84} & \multirow{2}{*}{83} & \multirow{2}{*}{81} & \multirow{2}{*}{81} & \multirow{2}{*}{82} & \multirow{2}{*}{81} & \multirow{2}{*}{80} & \multirow{2}{*}{79} & \multirow{2}{*}{79} & \multirow{2}{*}{79} & \multirow{2}{*}{77} & \multirow{2}{*}{79} & Class & uint8 \\
\hline & & & & & & & & & & & & Image type & intensity \\
\hline 84 & 84 & 84 & 82 & 81 & 79 & 78 & 78 & 79 & 79 & 78 & 79 & Minimum intensity & 20 \\
\hline 84 & 84 & 86 & 85 & 83 & 81 & 80 & 80 & 81 & 81 & 81 & 81 & Maximum intensity & 255 \\
\hline 84 & 84 & 87 & 86 & 84 & 83 & 81 & 81 & 82 & 83 & 85 & 82 & \multirow{2}{*}{\multicolumn{2}{|c|}{ Figure 47: Image Information }} \\
\hline 83 & 84 & 87 & 86 & 84 & 83 & 81 & 81 & 81 & 82 & 85 & 82 & & \\
\hline \multicolumn{14}{|l|}{1} \\
\hline «el & & & & & & & & & & & & & \\
\hline
\end{tabular}

Figure 46: Pixel Regions

Figure 47 displays the information or the details of the image. The width of the image represents how many columns of pixels are available in the image. This means, how many pixels are available in standard $\mathrm{X}$ direction. (The number of pixels in the horizontal direction is equal to the number of columns and number of columns equal to width of the image). Likewise, the height of the image is represented by the number of pixel rows that are available in vertical direction of the image. The class represents whether the image is unit8 or unit16. Image type shows whether the image is an intensity image or color (RGB) 
depending on the image's type. The minimum intensity and the maximum intensity attribute show the maximum and minimum intensity value that is available in the image.

Figure 48 shows the color histogram of the image selected. The Adjust Contrast tool opens in a separate window containing a histogram of the image displayed in the Image Viewer. The histogram shows the data range of the image and the display range of the image. The data range is the range of intensity values actually used in the image. The Adjust Contrast tool works by manipulating the display range and the data range of the image remains constant. To adjust the contrast of the image, the red-tinted rectangular box, called a window can be moved. By spreading out the intensity values as shown in Figure 48 an image with a better contrast can be obtained.

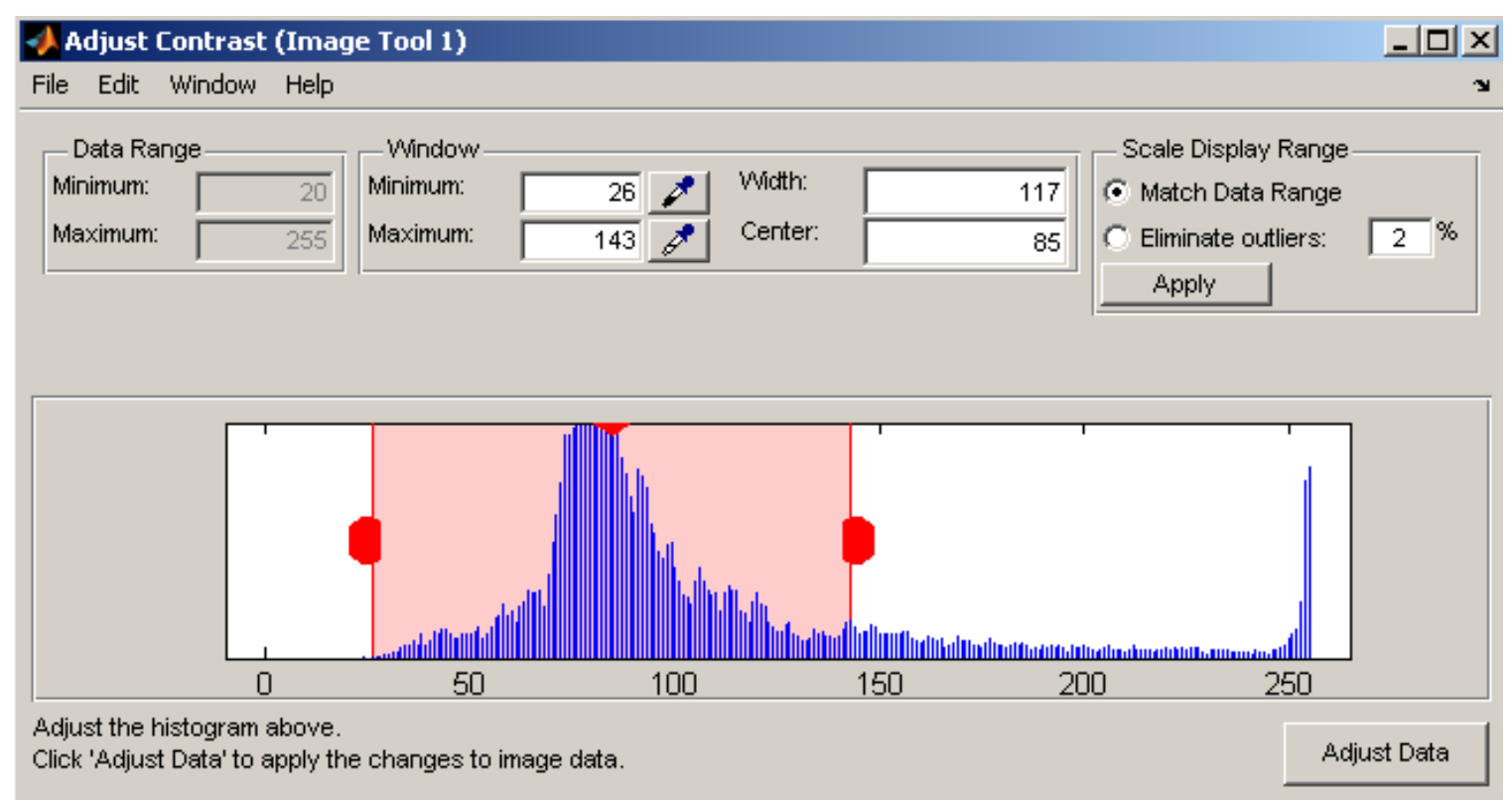

Figure 48: Adjusting the Contrast of the Image with the Histogram

In order to demonstrate the cropped true color images and the converted gray scale images, the montage function has been used. The montage function in Matlab allows one to display multiple images in other words "multi frame array" in a single figure. 
Figure 49 and Figure 50 display the ten successive cropped RGB and gray scale images respectively using the montage function. These images are useful in visualizing the bubble progression in the different frames. The code used to obtain such images is as follows:

montage(fileNames, 'Size', [2 5]);

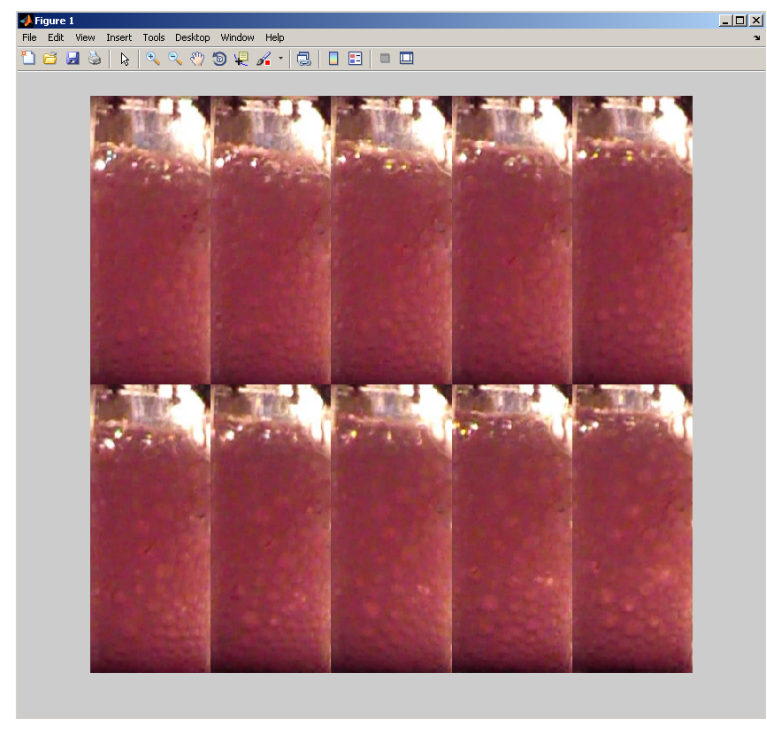

Figure 49: Consecutive Cropped Images in True Color

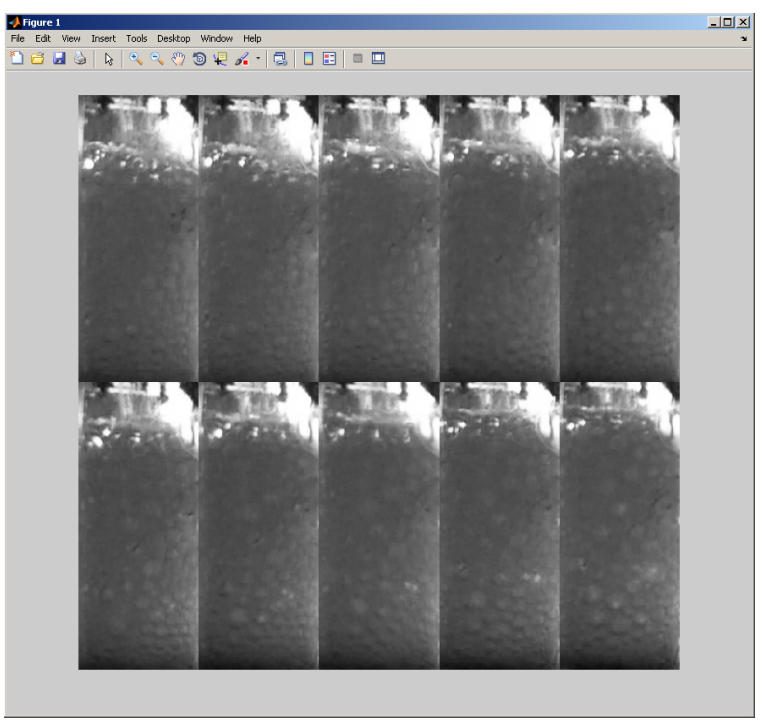

Figure 50: Consecutive Images in Gray Scale

As explained earlier, the "imcrop" command can be used manually using the mouse, or it is possible to assign the cropped image size as a rectangle by giving the pixel coordinate values where the rectangle needs to be created for cropping purposes, as illustrated below.

$$
\mathrm{D} 1=\operatorname{imcrop}\left(\mathrm{G} 1,\left[\begin{array}{llll}
150 & 25 & 150 & 360
\end{array}\right)\right. \text {; }
$$

In the above Matlab statement, D1 is the name for the image that has been cropped from the image G1. Within the square brackets in the Matlab statement, the first two numbers $(150,25)$ will demonstrate the minimum $\mathrm{X}$ coordinate pixel value and the minimum $\mathrm{Y}$ coordinate value, while the third and fourth numbers $(150,360)$ represent the width and the height of the rectangle. 


\section{Displaying Sequence of Images}

Using the montage function, the image frames that are in sequence can be shown as a movie in Matlab by making them display one after another and looping the entire process. The initial step will be storing images under the montage function in the Matlab workspace without losing or deleting any image that belongs to the sequence. Once the sequence has been created as explained, that sequence will be used with the immovie function. The Matlab immovie function can generate a movie clip of a sequence of images as it is recognized by the montage function. The following Matlab code demonstrates how to make a small video clip (movie) from a sequence of images.

$$
\begin{aligned}
& \text { montage (d) } \\
& \operatorname{mov}=\operatorname{immovie}(d) ; \\
& \operatorname{movie}(\mathrm{mov})
\end{aligned}
$$

\section{Obtaining Data}

In order to determine the $\mathrm{X}$ and $\mathrm{Y}$ coordinates of the center of a bubble, it is possible to use the Matlab "ginput" command. Once the "ginput" command is executed, it is possible to gain the X and Y coordinate values by clicking of the mouse button on the image that shows the bubble. Once the "ginput" command is used, the $\mathrm{X}$ coordinates values and $\mathrm{Y}$ coordinate values will appear as column vectors. This "ginput" command was very useful to determine the coordinate values of the bubbles accurately. Once the bubble coordinate locations had been clearly identified with respect to frame numbers, it was possible to determine the bubble path and vertical and horizontal velocities of the bubbles as they moved upwards along the tank. 


\begin{tabular}{|ll|}
\hline \multicolumn{2}{l|}{ imshow(D1) } \\
ginput() \\
ans $=$ \\
72 & 315 \\
66 & 295 \\
57 & 277 \\
53 & 265 \\
62 & 247 \\
70 & 244 \\
79 & 225 \\
91 & 204 \\
88 & 177 \\
79 & 156 \\
\hline
\end{tabular}

In the above code listing, the left hand column represents the $\mathrm{X}$ coordinates and the right hand side represents the $\mathrm{Y}$ coordinates values in pixels for the center of one specific bubble for ten consecutive frames. Each row will represent a single pair of coordinate points. As an example, $\mathrm{X}=72$ and $\mathrm{Y}=315$ for the first bubble center coordinate point that was picked by clicking the mouse button which can be written as $(72,315)$ in the common Cartesian coordinate mathematical notation.

\section{Importing Data from Excel Sheets to Matlab}

While gathering the bubble characteristic data and the information that appears in the images, it is quite convenient to document those data in Excel spreadsheets due to the simplicity of handling the spreadsheets. Even though it has been documented in the Excel sheets, those data needed to be imported into Matlab. The command "xlsread" was useful to import vast amounts of data points that are stored in multiple rows and columns in different spreadsheets. The Matlab statements shown below have been used to obtain the data points that were documented in the "pb36.xlsx" Excel sheet from the sheet number in the column assigned with " $R$ " that started from the row number 6 to the row number 51 . Once the data was imported into Matlab, it was easy to conduct the necessary additional Matlab operations to obtain the characteristics information of the bubbles, such as bubble diameter or bubble velocity. 


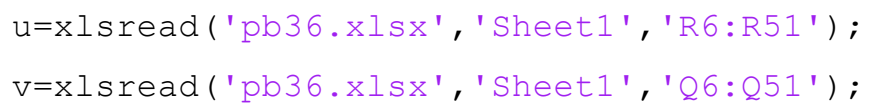

\section{Results}

By comparing the horizontal radius and vertical radius of the bubble at the bottom of the tank to the top of the tank, we were able to determine the variations of the bubble's size and geometric shape. At the bottom of the tank, the bubble's vertical radius is comparatively smaller than the horizontal radius. When the bubble is at the bottom of the tank, it goes through the strongest region of the magnetic field. As the bubble goes upwards along the tank, the strength of the magnetic field reduces and eventually the vertical component of the radius gets bigger than the horizontal radius. Later, at the beginning of the upper half region of the tank, the vertical component of the bubble radius dominates.

When the bubbles reach more towards the top of the tank (approximately three quarters of the height of the tank from the bottom), again the bubble's horizontal and vertical components of the radius values get more close to one another. At the top of the tank, the magnetic field strength is very weak, and the bubble's shape demonstrates a nearly circular shape with almost equal horizontal and vertical radius. Location of the $\mathrm{X}$ coordinate versus each relevant frame number that has constant frame rate has been graphed to demonstrate the bubble path. While the bubble travels upwards, bubble movement demonstrates a 2-D oscillating path along the tank as shown in Figure 51.

The first three frames with the bubble were omitted to determine the bubble center location, vertical and horizontal radius of the bubble due to poor visibility since it was very close to the boiling heater. Therefore, for the accuracy, characteristic study of the bubbles has begun from the fourth frame. 


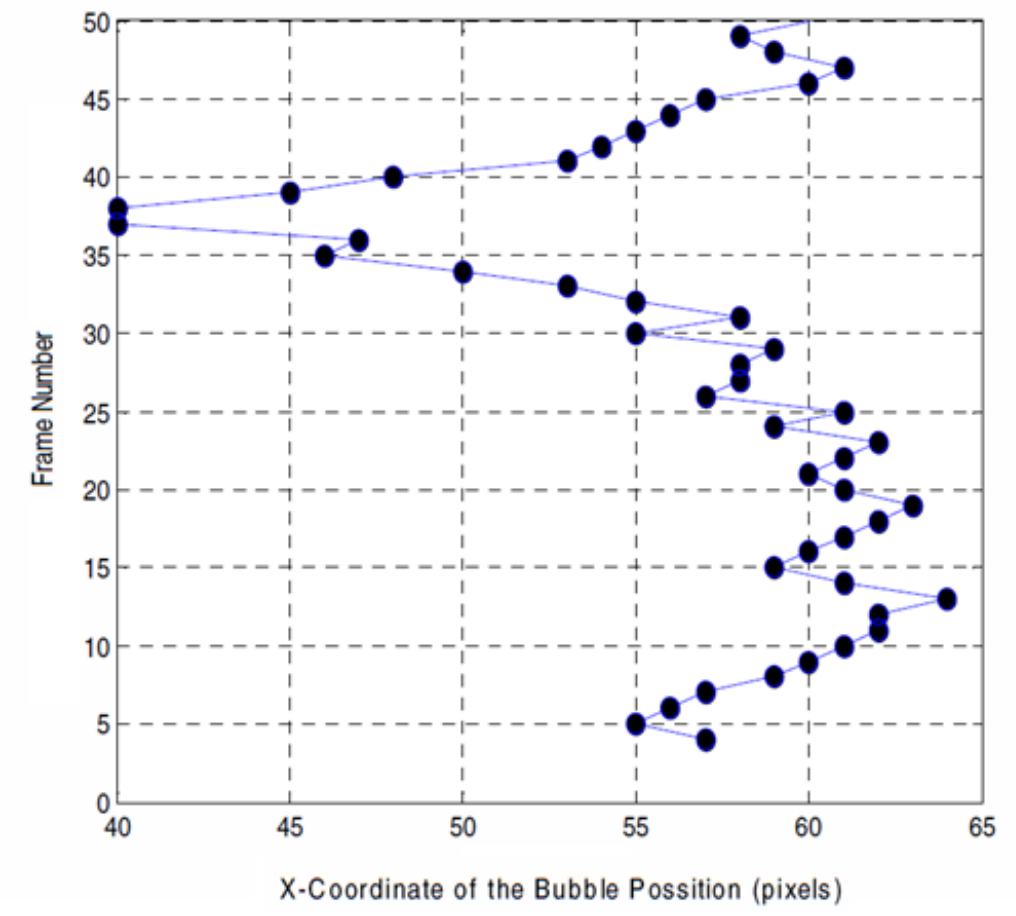

\section{Figure 51: The Two Dimensional Oscillating Path Behavior of the Bubble as it Travers up the Tank in the Magnet Tank}

Between frame numbers 10 to 30, bubble's motion path demonstrates the smooth 2-D oscillating path and after the $31^{\text {st }}$ frame, the bubble has lost its smoothness in the 2-D path and it was pushed more towards to the left side of the tank. As we can see, when the bubble travels in the upper region of the tank with low magnetic field strength, with respect to the frame numbers 30 and above, the bubble has lost its pattern in oscillating path behavior. Figure 52 shown below demonstrates the location of the bubble's Y coordinate along the vertical axis of the tank. The bubble moves more quickly as it approaches the top of the tank and that can be visualized by observing the gap between data points at the top end of the graph relevant to frame number from 40 to 50 . Also, the vertical bubble path coordinate is much smoother than the original path coordinate as can be seen in Figure 51. 


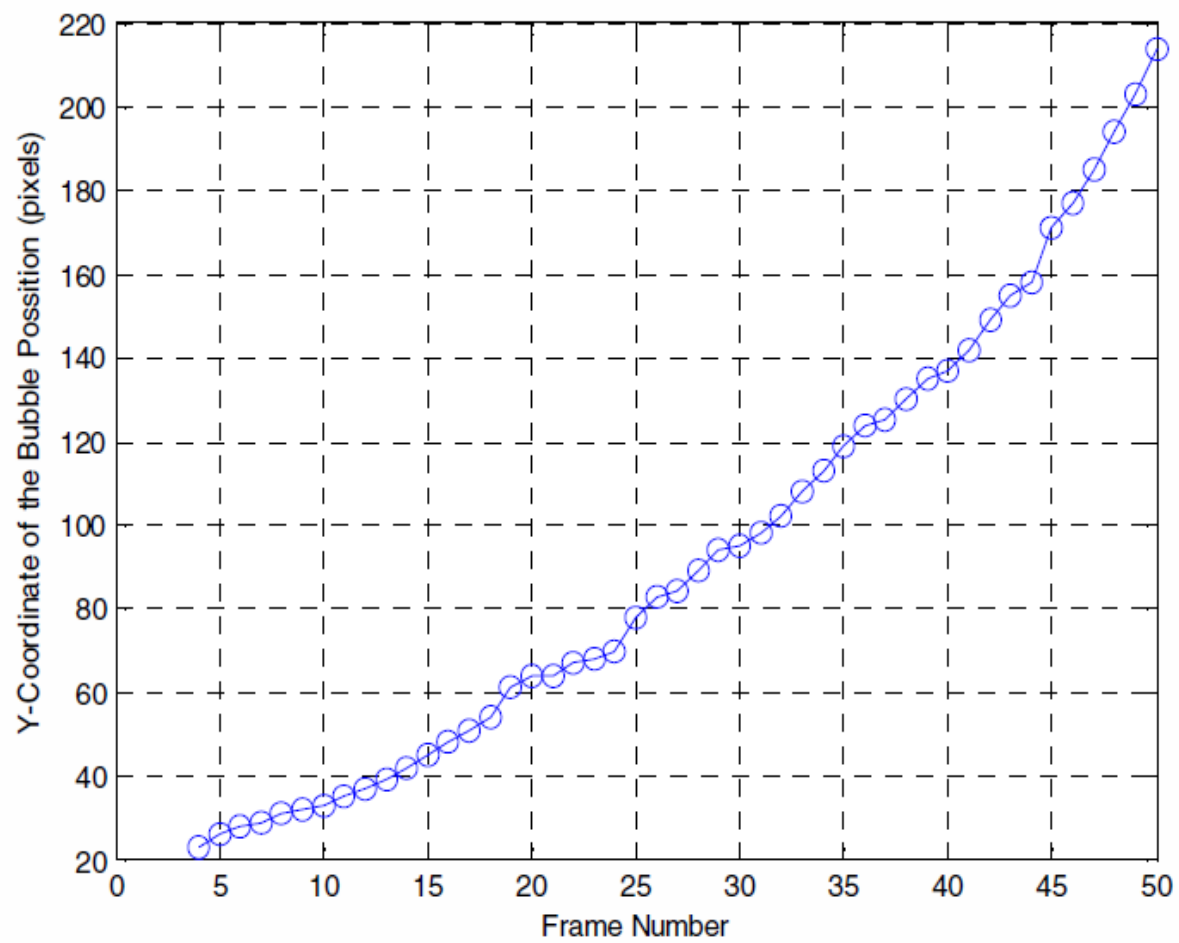

Figure 52:The Vertical Displacement of the Center of the Bubble's As Ii Travers up Long the Magnet Tank

Figure 53 demonstrates the values of the horizontal and vertical radius in pixels with respect to the frame number. As the bubble rises along the travel path, size of the bubble increases. The bubble size can be determined by evaluating the bubble's horizontal radius and vertical radius with respect to its location. The measured bubble radii are shown in Figure 53, again plotted verses the frame number. 


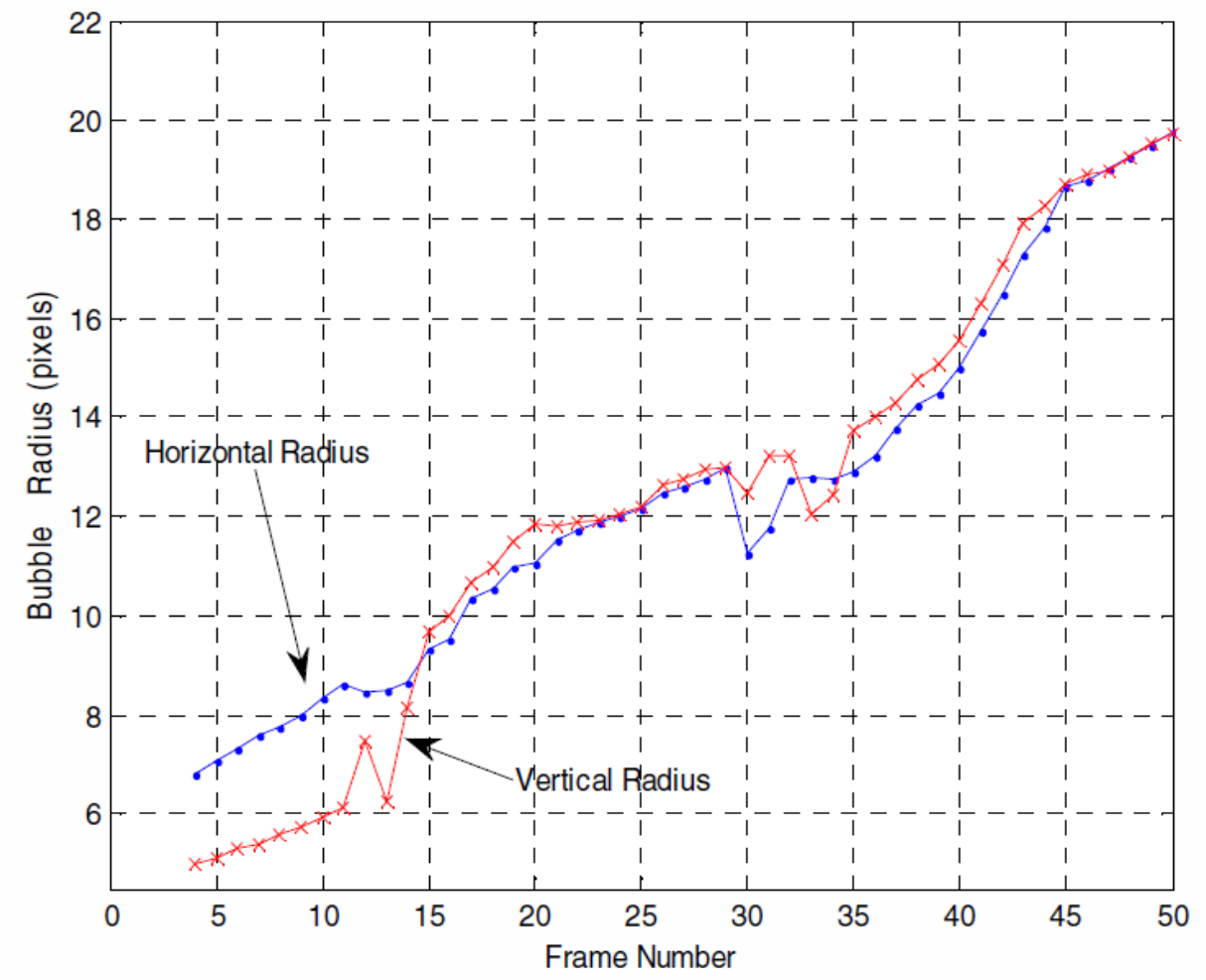

Figure 53: Difference in the Horizon and Vertical Radii (semi major and semi minor axes) of the Bubble as it Travers up the Tank Under the Influence of the Magnetic Field

Figure 54 displays the computed projected area of the bubble versus the frame number shown in pixel-squared. When the bubble moves along the vertical axis, both the horizontal and vertical radius of the bubble were increasing and as a result of that, the area of the bubble was observed to increase along the vertical axis of the boiling tank. 


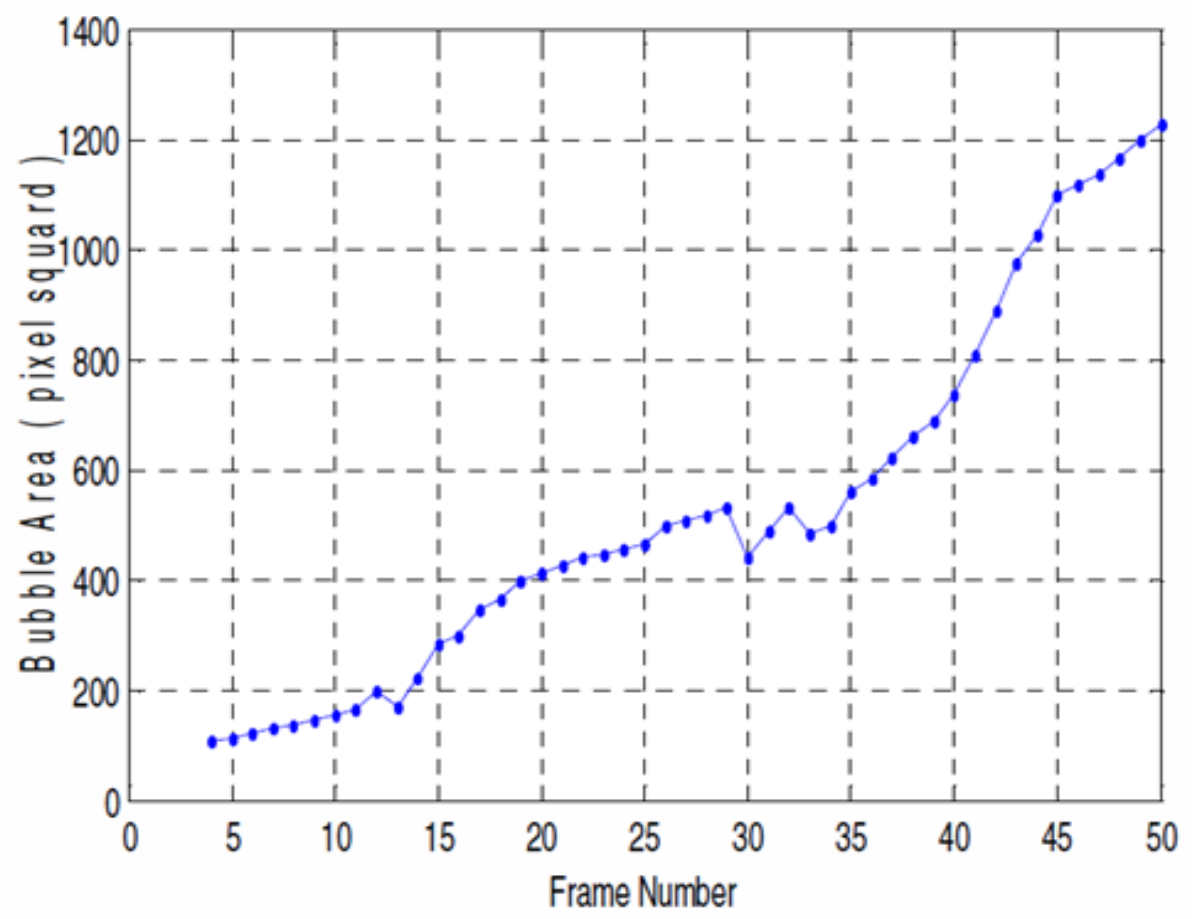

\section{Figure 54: The Visualization of the Increase of the Bubble Area as it Travers up Along the Magnet Tank}

Figure 55 demonstrates the computed velocity vector history with respect to the location along the vertical $\mathrm{Y}$-axis of the tank. Length of the arrow demonstrates the magnitude of the average velocity $V_{\mathrm{A}}$ that was calculated from the horizontal velocity (u pix/sec) component and the vertical velocity component ( $\mathrm{v}$ pix/sec) and direction of the arrow demonstrate the direction of the average velocity vector in pixels/second at a given particular location on the coordinate system. 


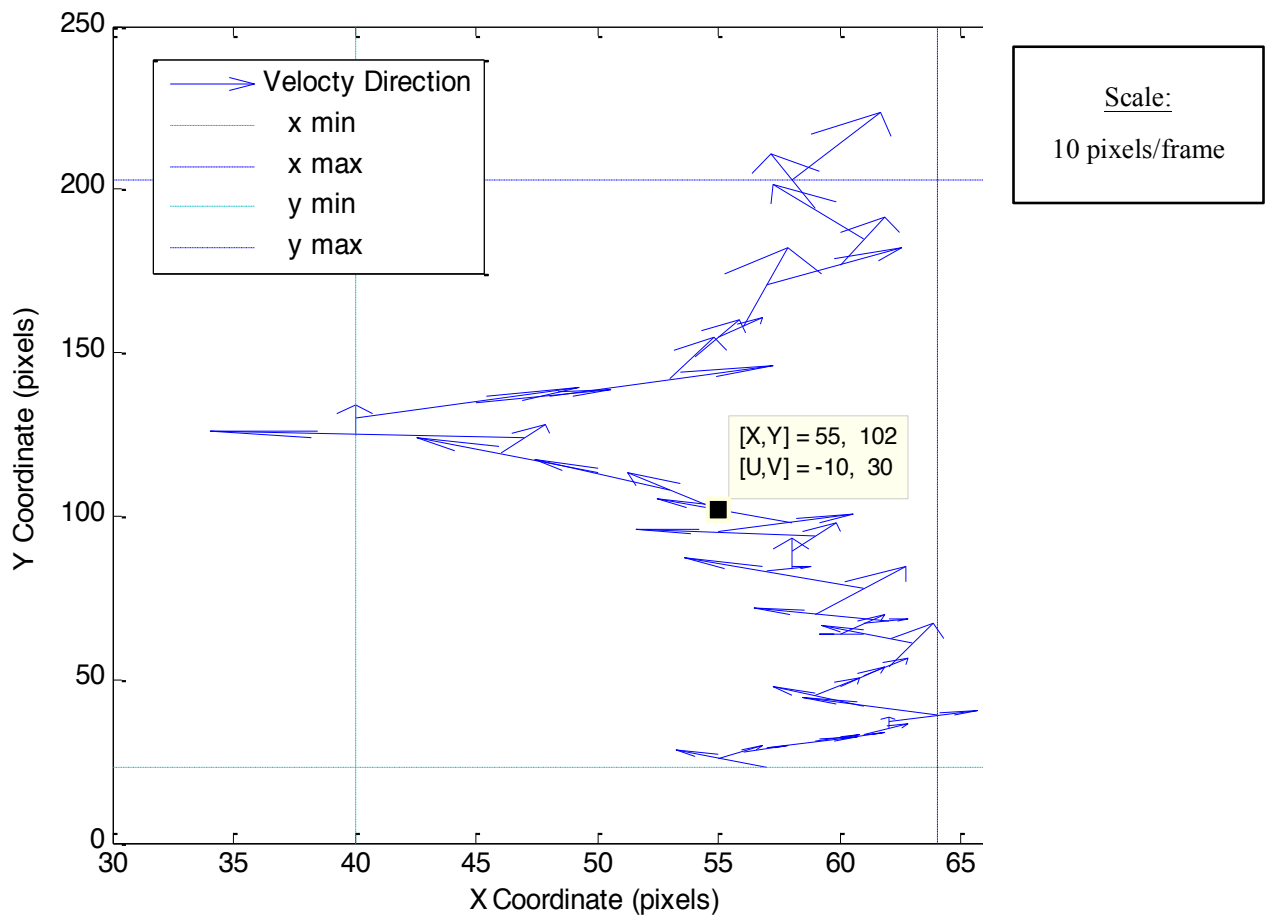

Figure 55: Resultant Velocity Vector of the Horizontal Velocity Component and the Vertical Velocity Component of the Bubble with Respect to the Position as it Traverse Along the Magnet Tank 


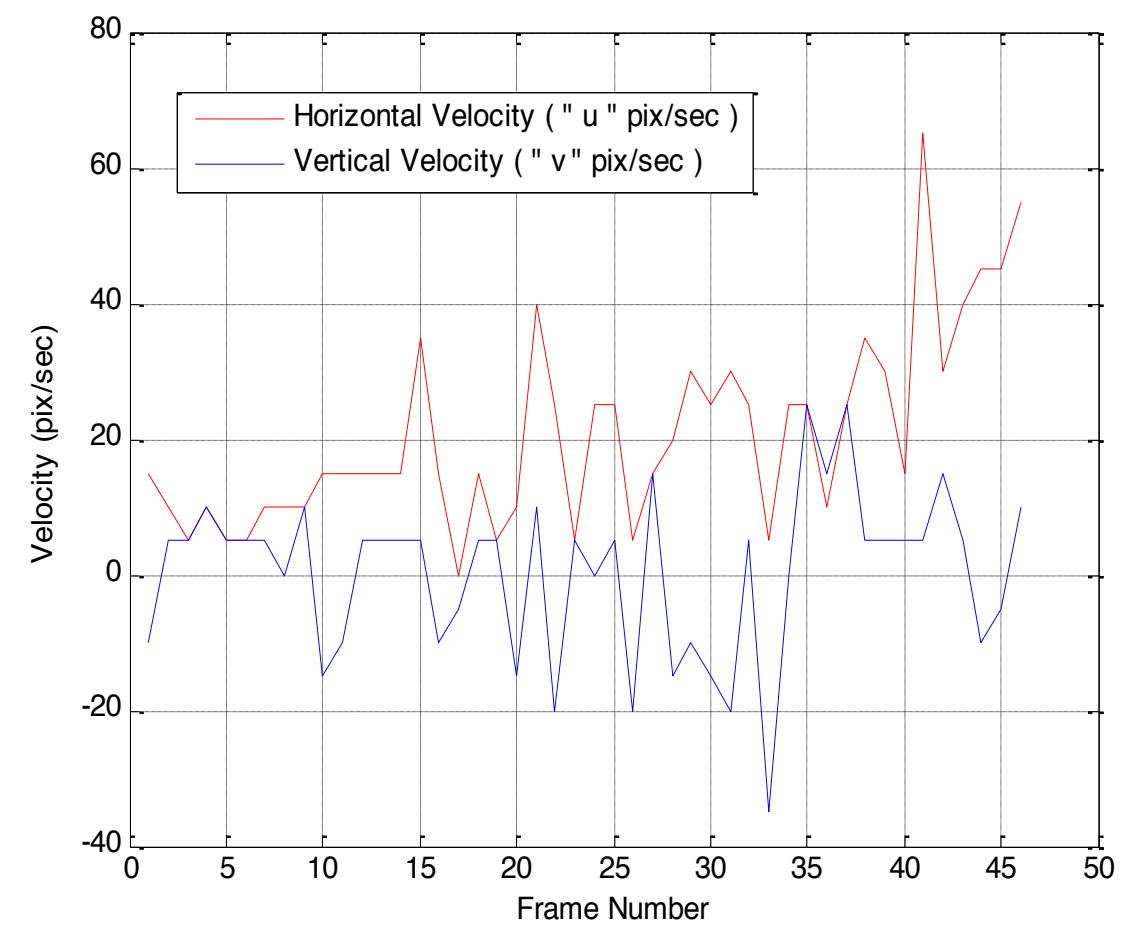

Figure 56: The Visual Representation of the Fluctuations in Vertical and Horizontal Velocity Components as the Bubble Travers upward the Tank

The next step was to compare the bubbles in the two tanks. During this process, one bubble was chosen from each tank at the same height level from the bottom of the tank in order to conduct the comparison analysis. The two bubbles from each tank were chosen comparatively in the similar regions in their height from the bottom of the tank. One of the difficulties faced during this selection process was to find bubbles that go through from the bottom of the tank to the top of the tank continuously without disappearing while comparing them to the chosen bubble from the other tank. It was difficult in practice to determine a two-bubble set in each consecutive frame with the time gap of 0.2 seconds.

After careful analysis, it was possible to recognize a pattern from every other frame that had two consecutive bubble sets from each tank with respect to the same height level from the bottom tank to the top of the tank. Therefore, a special case study has been done with the time gap of 0.4 seconds in consecutive frames from the video clip, which is evaluating every other image frame from the 0.2 seconds frame sequence. 
The objective of conducting a comparative analysis study of the available characteristics of the two selected bubbles from each tank was to determine what significant influences the magnetic field on the bubble during the pool boiling and what those influences have implied on bubble behavior as they move along the tank during the boiling period under microgravity environment.

Table 6 illustrates the data obtained from conducting an image processing technique that was discussed earlier for studying the characteristics of the bubble chosen from the tank with the magnet.

Table 6: Characteristic Data of a Bubble from the Tank with the Magnet

\begin{tabular}{|c|c|c|c|c|c|c|c|c|c|c|c|c|}
\hline & & $X$-Coordinate & $X$-Coordinate & $X$-Coordinate & Y-Coordinate & Y-Coordinate & Y-Coordinate & & & & & \\
\hline Original Image & & Left & Right & Center & Upper & Lower & Center & Horizontal & Vertical & Horzontal & Vertical & Average \\
\hline Number & Frame Number & $X L$ & $X R$ & $X C$ & $Y u$ & YL & $Y_{c}$ & Diameter & Diameter & Radius & Radius & Radius \\
\hline 20 & 1 & 81 & 95 & 88 & 316 & 331 & 321 & 14 & 15 & 7 & 7.5 & 7.25 \\
\hline 22 & 2 & 76 & 95 & 86 & 313 & 328 & 321 & 19 & 15 & 9.5 & 7.5 & 8.5 \\
\hline 24 & 3 & 81 & 97 & 89 & 301 & 322 & 317 & 16 & 21 & 8 & 10.5 & 9.25 \\
\hline 26 & 4 & 80 & 99 & 89 & 298 & 315 & 307 & 19 & 17 & 9.5 & 8.5 & 9 \\
\hline 28 & 5 & 89 & 107 & 97 & 283 & 303 & 294 & 18 & 20 & 9 & 10 & 9.5 \\
\hline 30 & 6 & 91 & 110 & 100 & 271 & 292 & 281 & 19 & 21 & 9.5 & 10.5 & 10 \\
\hline 32 & 7 & 90 & 107 & 99 & 261 & 283 & 269 & 17 & 22 & 8.5 & 11 & 9.75 \\
\hline 34 & 8 & 95 & 121 & 109 & 240 & 262 & 232 & 26 & 22 & 13 & 11 & 12 \\
\hline 36 & 9 & 93 & 127 & 105 & 235 & 267 & 232 & 34 & 32 & 17 & 16 & 16.5 \\
\hline 38 & 10 & 95 & 131 & 97 & 217 & 252 & 218 & 36 & 35 & 18 & 17.5 & 17.75 \\
\hline 40 & 11 & 90 & 133 & 111 & 201 & 245 & 174 & 43 & 44 & 21.5 & 22 & 21.75 \\
\hline 42 & 12 & 89 & 135 & 100 & 194 & 241 & 143 & 46 & 47 & 23 & 23.5 & 23.25 \\
\hline 44 & 13 & 87 & 138 & 116 & 187 & 237 & 98 & 51 & 50 & 25.5 & 25 & 25.25 \\
\hline
\end{tabular}

Table 7 illustrates the characteristics of the bubble that was chosen from the tank without the magnet. 
Table 7: Characteristic Data from the Tank without the Magnet

\begin{tabular}{|c|c|c|c|c|c|c|c|c|c|c|c|c|}
\hline & & X-Coordinate & X-Coordinate & X-Coordinate & Y-Coordinate & Y-Coordinate & $Y$ - Coordinate & & & & & \\
\hline Original Image & & Left & Right & Center & Upper & Lower & Center & Horizontal & Vertical & Horzontal & Vertical & Average \\
\hline Number & Frame Number & XL & $X R$ & $x_{c}$ & Yu & YL & $Y_{c}$ & Diameter & Diameter & Radius & Radius & Radius \\
\hline 20 & 1 & 313 & 328 & 322 & 319 & 332 & 325 & 15 & 13 & 7.5 & 6.5 & 7 \\
\hline 22 & 2 & 327 & 343 & 336 & 311 & 326 & 320 & 16 & 15 & 8 & 7.5 & 7.75 \\
\hline 24 & 3 & 305 & 328 & 317 & 303 & 321 & 315 & 23 & 18 & 11.5 & 9 & 10.25 \\
\hline 26 & 4 & 327 & 352 & 335 & 299 & 320 & 309 & 25 & 21 & 12.5 & 10.5 & 11.5 \\
\hline 28 & 5 & 356 & 385 & 371 & 282 & 307 & 296 & 29 & 25 & 14.5 & 12.5 & 13.5 \\
\hline 30 & 6 & 356 & 390 & 374 & 267 & 296 & 280 & 34 & 29 & 17 & 14.5 & 15.75 \\
\hline 32 & 7 & 360 & 395 & 378 & 251 & 280 & 268 & 35 & 29 & 17.5 & 14.5 & 16 \\
\hline 34 & 8 & 393 & 427 & 407 & 217 & 247 & 232 & 34 & 30 & 17 & 15 & 16 \\
\hline 36 & 9 & 316 & 337 & 324 & 212 & 230 & 226 & 21 & 18 & 10.5 & 9 & 9.75 \\
\hline 38 & 10 & 346 & 368 & 346 & 201 & 223 & 214 & 22 & 22 & 11 & 11 & 11 \\
\hline 40 & 11 & 348 & 379 & 364 & 173 & 207 & 192 & 31 & 34 & 15.5 & 17 & 16.25 \\
\hline 42 & 12 & 356 & 418 & 386 & 119 & 171 & 147 & 62 & 52 & 31 & 26 & 28.5 \\
\hline 44 & 13 & 344 & 412 & 378 & 57 & 113 & 84 & 68 & 56 & 34 & 28 & 31 \\
\hline
\end{tabular}

Figure 57 demonstrates the size of the vertical and horizontal radius in pixels relevant to their frame number for the bubble that was under the influence of the magnetic field. 


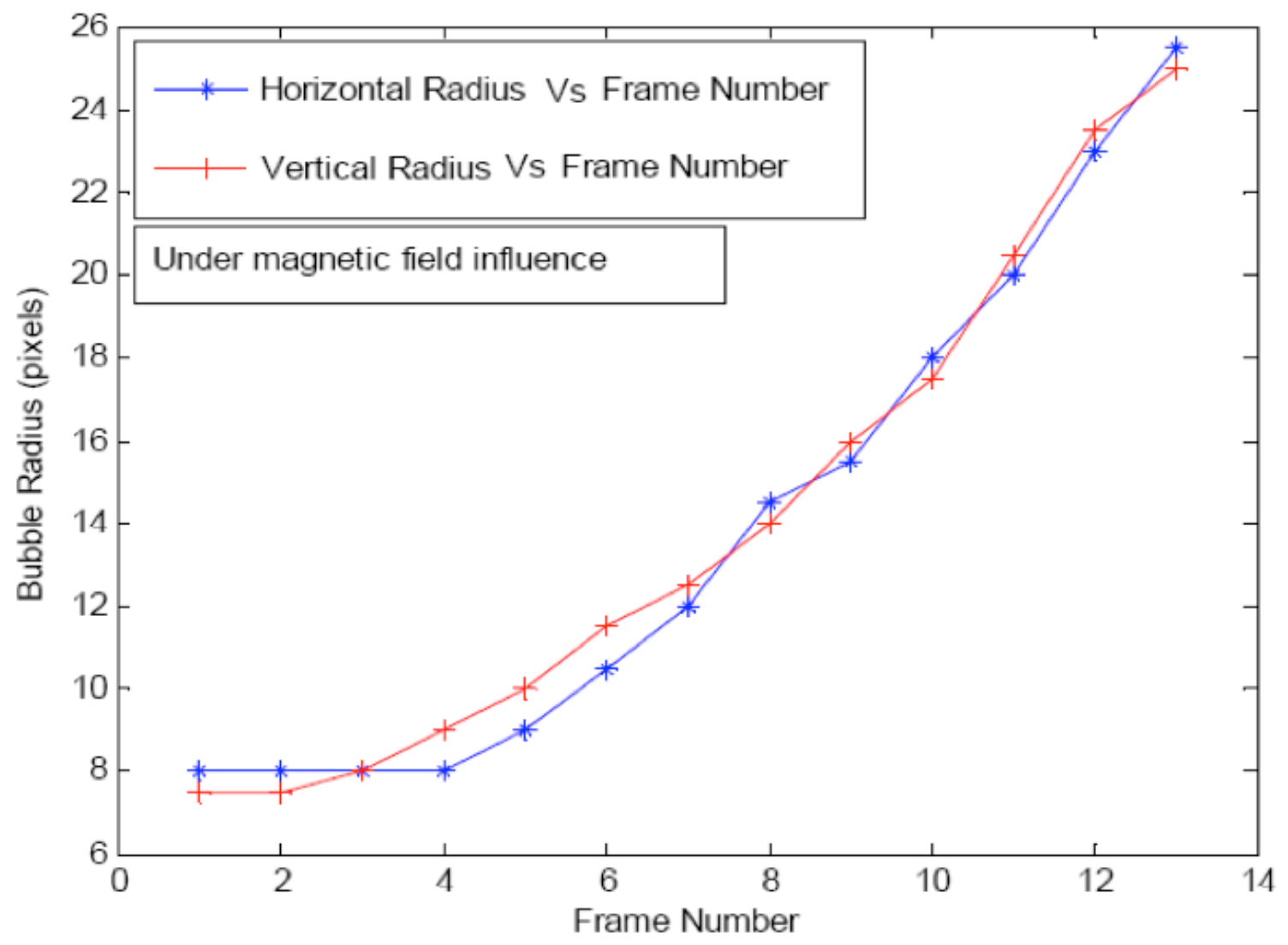

Figure 57: Horizontal and Vertical Radius of the Bubble vs. Frame Number under Magnetic Field

Figure 58 demonstrates the size of the bubble's vertical and horizontal radius in pixels relevant to their frame number without the influence of the magnetic field. As demonstrated in both the figures above, the horizontal and vertical radii of the bubbles under the influence of magnetic field are very close to each other. In other words, under the magnetic field influence, bubbles which were generated by pool boiling under the microgravity conditions are more circular in their cross sectional shape, compared to the bubbles generated without the magnetic force. 


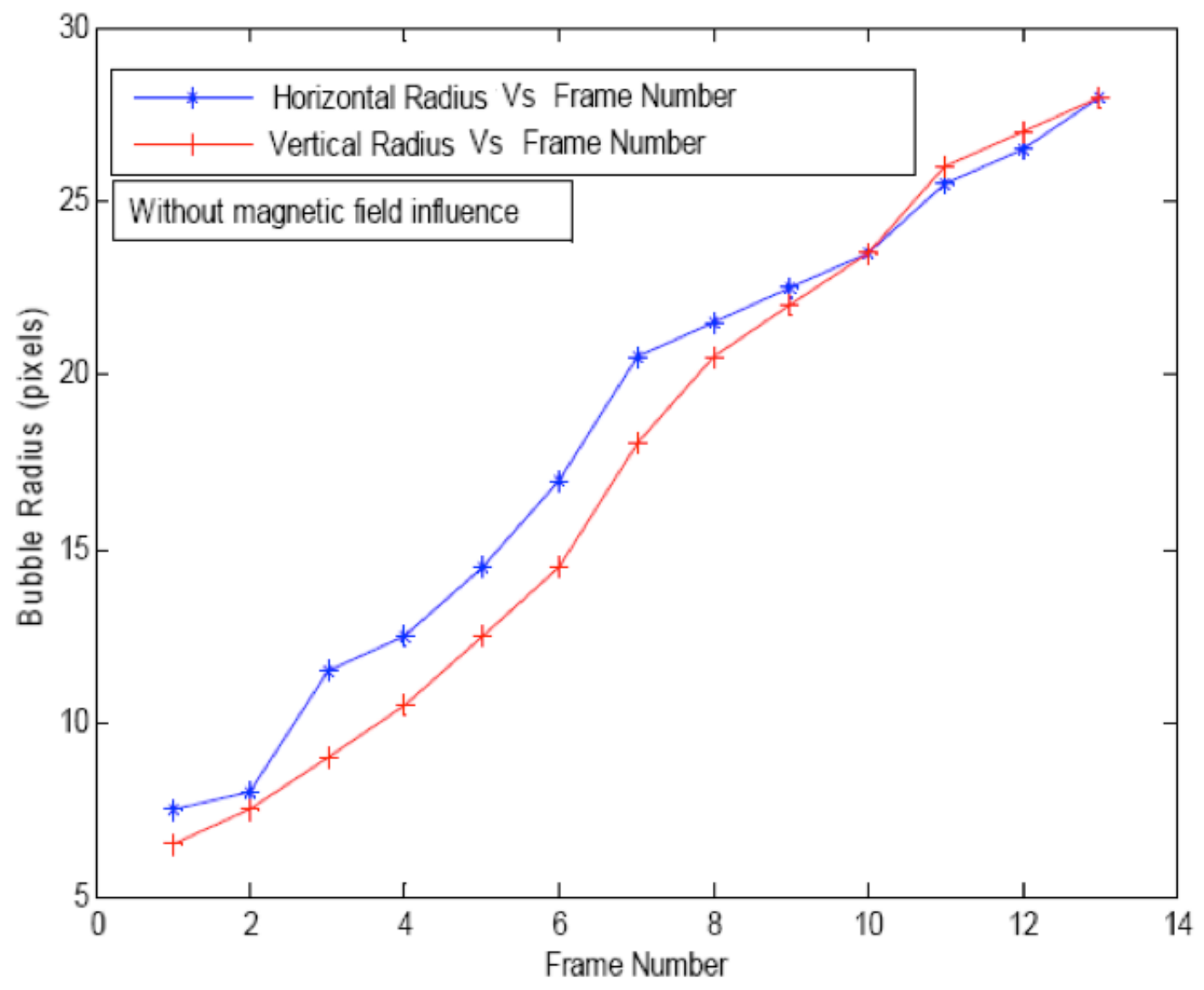

Figure 58: Visual Representation of the Size Increase of the Horizontal and Vertical Radii of the Bubble located in the Non-Magnet Tank as it Traverse up the Tank

Figure 59 demonstrates the calculated area of the bubbles with respect to the frame number with and without the influence of the magnetic field, in which average radius was used to calculate area of the bubble. The bubble under the influence of the magnetic field is consistently significantly smaller than the bubble in the tank without the magnet, even though the two bubbles initially were nearly the same size. 


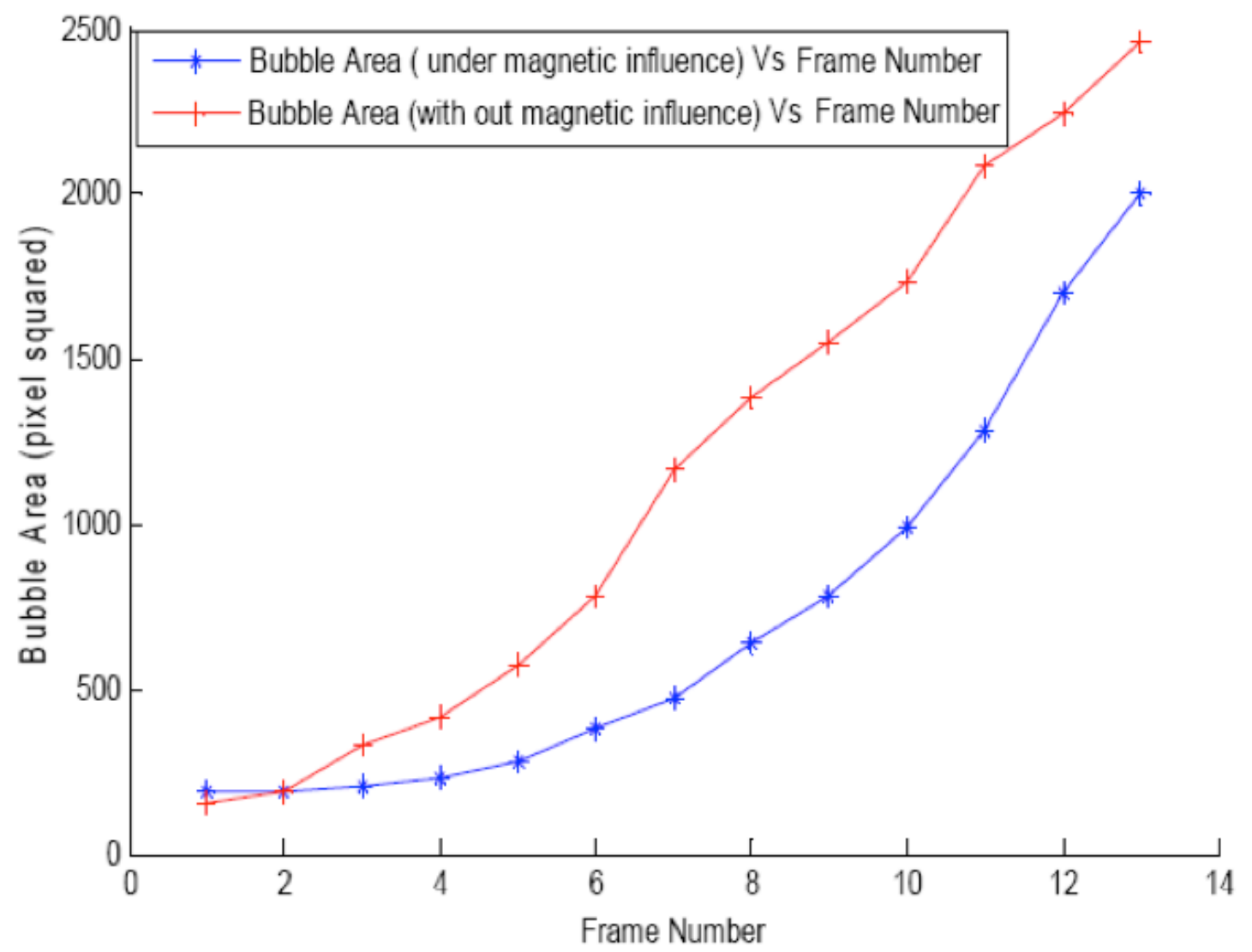

Figure 59: Representation of the Increase in Area of Two chosen Bubbles, one Bubble Under the Influence of the Magnetic Field and the Other Bubble, without the Influence of the Magnetic Field as they Traverse Upward the Tank 


\section{References}

Andres, U. T. "Magnetic Liquids" Materials Science and Engineering, Vol. 26, 1976, pp 269-275.

Di Marco, P, and Grassi W. 1997. "Nucleate Pool Boiling in the Presence of an Electric Field: Effect of Subcooling and Heat-up Rate.” Experimental Thermal and Fluid Science 15 (3): 213-20.

Di Marco, P. 2010. "Influence of Force Fields and Flow Patterns on Boiling Heat Transfer Performance." In 2010 14th International Heat Transfer Conference, Volume 8. doi:10.1115/ihtc14-23409.

Di Marco, P, and Grassi W. 2007. "Pool Boiling in Microgravity and in Electrical Fields: Old and Recent Results." Multiphase Science and Technology 19 (2): 141-65.

Di Marco, P, and Grassi W. 2009. "Pool Boiling In Microgravity And In The Presence Of Electric Field: Evaluation Of The Void Fraction In The Ariel Experiment." Multiphase Science and Technology 21 (4): 267-77.

Di Marco, P, and Grassi W. 2007. "Effect of Force Fields on Pool Boiling Flow Patterns in Normal and Reduced Gravity." Heat and Mass Transfer 45 (7): 959-66.

Di Marco, P., R. Raj, and J. Kim. 2011. "Boiling in Variable Gravity under the Action of an Electric Field: Results of Parabolic Flight Experiments.” Journal of Physics. Conference Series 327: 012039.

Gabriel, K. S. Microgravity Two-phase flow and heat transfer. New York: Springer, 2007.

Gonzalez, R C., and Woods, R.E. "Digital image processing". New Jersey: Prentice-Hall, 2002.

Ferreira, T, and Wayne R. 2012. ImageJ User Guide: IJ 1.42r.

Kim, J., Benton, J., Mcquillen, J., and Vickerman, M. 2001. "Subcooled Pool Boiling Heat Transfer In Microgravity And Hi-G.” Journal of Heat Transfer 123 (4): 620.

Kubota, C., O. Kawanami, Y. Asada, Y. Wada, T. Nagayasu, Y. Shinmoto, H. Ohta, et al. 2011. "Experiment on Nucleate Pool Boiling in Microgravity by Using Transparent Heating Surface - Analysis of Surface Heat Transfer Coefficients." Journal of Physics. Conference Series 327: 012040.

Kuhlman, J, and Gray, D, Glaspell, S, and Kreitzer, P. "Positioning of Bubbles in Microgravity by the Kelvin Force." Paper AIAA 5201 (2005): 6-9.

Kuhlman, J, and Gray, D. "Effects of the Magnetic Kelvin Force on Pool Boiling in Microgravity." Paper AIAA 4268 (2007).

Madhavan, V., V. A. Gandikota, R. Agarwal, Ho S. Lee, Merte Herman, and Chiaramonte Francis. 1997. "Pool Boiling Curve in Microgravity." Journal of Thermophysics and Heat Transfer 11: 216-22.

Mahajan, M. P, Tsige, M., Taylor, P. L., and Rosenblatt, C. "Paramagnetic liquid bridge in a gravitycompensating magnetic field." Physics of Fluids 10 (1998): 2208-2211.

Oka, T., Abe, Y., Mori, Y.H., and Nagashima, A. 1995. "Pool Boiling of N-Pentane, CFC-113, and Water Under Reduced Gravity: Parabolic Flight Experiments With a Transparent Heater." Journal of Heat Transfer 117 (2): 408-417.

Sanada, T., Watanabe, M., and Fukano, T., 2003: "Study on Single Bubble Chains in Stagnant Water", Proc. ASME FEDSM03, 4th Joint ASME JSME Fluids Engineering Conference, Honolulu, Hawaii, July 6-10.

Straub, J. 2001. "Boiling Heat Transfer and Bubble Dynamics in Microgravity." In Advances in Heat Transfer, 57-172. 
Wakayama, N. I., H.Ito, Y.Kuroda, O.Fujita \& K.Ito, Magnetic support of combustion in diffusion flames under Microgravity, Combustion \& Flame, 107, 187 (1996); J. Japan Soc. Microgravity Applications, 11,268( 1994).

Wakayama, N.I., Magnetic buoyancy force acting on bubbles in nonconducting and diamagnetic fluids under microgravity, JAppl.Phys., 81,2980 (1997); ./. Japan Soc. Microgravity Applications, 12,279 (1995); ibid., 13, 369 (1996).

White, F. M., Viscous Fluid Flow, 2nd Edition, McGraw-Hill, 1991, pp. 177-183.

Zhao, J. F., S. X. Wan, G. Liu, Z. D. Li, and W. R. Hu. 2007. "Pool Boiling Heat Transfer in Microgravity." Microgravity Science and Technology 19 (3-4): 135-36.

Xue, Y. F., Zhao, J. F., Wei, J. J., Li, J., Guo, D., \& Wan, S. X. (2011). Experimental study of nucleate pool boiling of FC-72 on smooth surface under microgravity. Microgravity Science and Technology, 23(1), 75-85.

“Themal-FluidCentral." Webpage. Accessed April 27 2016.

https://www.thermalfluidscentral.org/encyclopedia/index.php/Pool_Boiling_Regimes. 